University of San Diego

Digital USD

2014-08-01

\title{
The Role of Chief Diversity Officers in Institutionalizing Diversity and Inclusion: A Multiple Case Study of Three Exemplar Universities
}

Cynthia D. Dávalos PhD

University of San Diego

Follow this and additional works at: https://digital.sandiego.edu/dissertations

Part of the Leadership Studies Commons

\section{Digital USD Citation}

Dávalos, Cynthia D. PhD, "The Role of Chief Diversity Officers in Institutionalizing Diversity and Inclusion: A Multiple Case Study of Three Exemplar Universities" (2014). Dissertations. 853.

https://digital.sandiego.edu/dissertations/853 
THE ROLE OF CHIEF DIVERSITY OFFICERS IN INSTITUTIONALIZING DIVERSITY AND INCLUSION:

A MULTIPLE CASE STUDY OF THREE EXEMPLAR UNIVERSITIES

by

Cynthia D. Dávalos

A dissertation submitted in partial fulfillment

of the requirements for the degree of

Doctor of Philosophy

August 2014

Dissertation Committee Cheryl Getz, Ed.D., Chair Lea Hubbard, Ph.D., Member

Frank Harris III, Ed.D., Member

University of San Diego 
(C) Copyright by Cynthia D. Dávalos

All Rights Reserved 2014 


\begin{abstract}
Due to demographic shifts and the changing political and economic landscape, universities are experiencing increased demands to produce a culturally competent and well-trained globally minded workforce. To address these demands in a systematic manner, several universities have created a new senior level administrative position to direct campus diversity and inclusion efforts. This position known universally in academia as the Chief Diversity Officer (CDO) is responsible for institutionalizing diversity and inclusion so that diversity moves from the margins of the university to the center and becomes a standard way of thinking and doing business. Given this high-level executive leadership design, the functions and duties of the CDO are significant. A CDO must be the advocate and leader for sustained institutional change that supports diversity and inclusion campus-wide. Despite the rise of these new positions, little empirical evidence exists about the role CDOs play in making campuses more diverse, inclusive, and equitable places to study, live, work, and teach.

This multiple case qualitative study examines three exemplar universities (a private faith-based West Coast institution, a large Midwestern public research university, and a large Southern public research university) who have a CDO that has experienced success at institutionalizing diversity and inclusion into the greater campus culture. Three research questions guided this study: (a) What role do CDOs play in institutionalizing diversity and inclusion in US universities; (b) How do the universities know they have institutionalized diversity and inclusion? What are their outcome measures and who is involved in these change efforts; and (c) What key strategies
\end{abstract}


contribute to successful institutional efforts to sustain diversity and inclusion? What is the role of the CDO in these efforts?

This study suggests that universities can institutionalize diversity and inclusion with a $\mathrm{CDO}$ who is committed to leading change through a systemic, relational, and integrated approach. Through intentional efforts that include relationship building, trust, and patience for a lengthy transformational change process, CDOs can help institutionalize diversity and inclusion so that it is a sustainable and permanent condition that positively affects the way a university functions and makes decisions. 


\section{DEDICATION}

It is fitting that the last two chapters of this dissertation were written at the home I grew up in and where my parents still live. From grade school to the completion of this dissertation my parents have been behind me cheering me on; first to leave home to go to college, first to receive a master's degree, and first to pursue a Ph.D. Because of their love, this dissertation and all of my educational achievements are dedicated to my mother and father, Manuel and Emma Dávalos. Without their encouragement to aim high and unwavering support I would not be the person I am today.

I also dedicate this dissertation and all of my education to my paternal and maternal grandparents who are deceased but not forgotten. As immigrants they left Mexico and arrived in the US with grade school educations and a work ethic like no other. Through their commitment to family, faith, and hard work, their dreams have been realized one more time in me. Although they are not present to witness this accomplishment, I recognize their contributions. I do not take for granted the privileges their sacrifices or my parents' have afforded me. I know whose shoulders I stand on and I do so with immense pride and gratitude. 


\section{ACKNOWLEDGEMENTS}

I would like to express deep gratitude to my dissertation committee who challenged and supported me throughout this journey. I would also like to acknowledge the faculty at the School of Leadership and Education Sciences at the University of San Diego who have made me a more intelligent, competent, and compassionate leader. Their offerings and support as well those of my fellow classmates made this investment of time and energy worthwhile. In addition, I wish to acknowledge the support of my professional colleagues who were patient and kind with me as I worked fulltime and managed a doctoral program. You cheered me on when I griped about how difficult it was to juggle both and reassured me this was only temporary. Last but never least, I wish to recognize my beloved family and friends who supported me from day one. Your reminders that my commitment would pay off in ways seen and unseen was certainly true and most comforting when I could not spend as much time with you as I wanted. I love you all and can never thank you enough for your support. 


\section{TABLE OF CONTENTS}

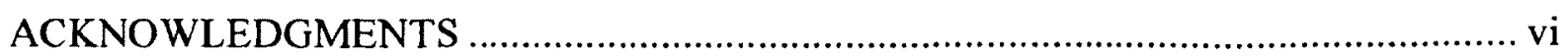

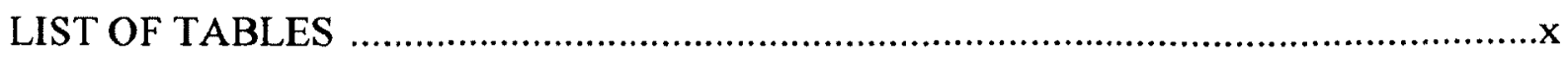

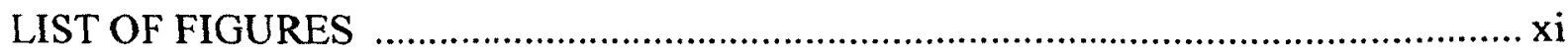

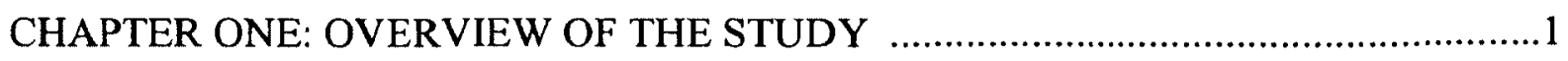

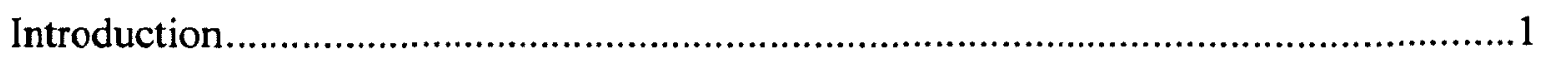

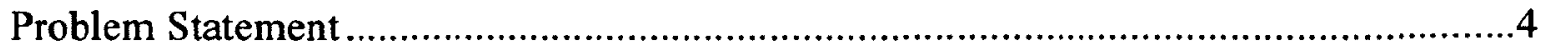

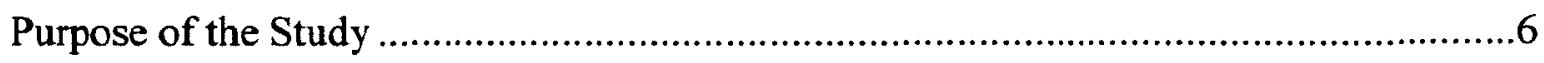

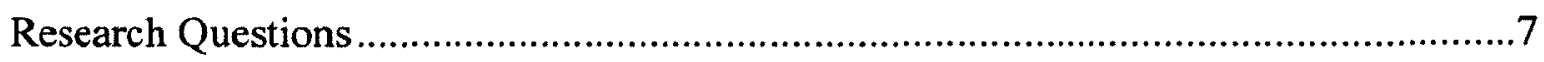

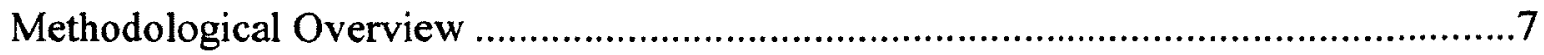

Significance of the Study ......................................................................................

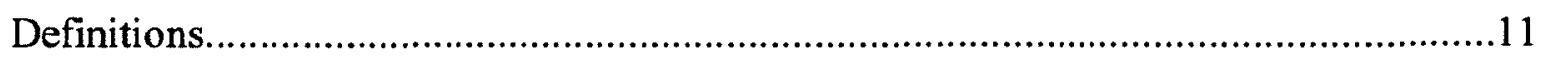

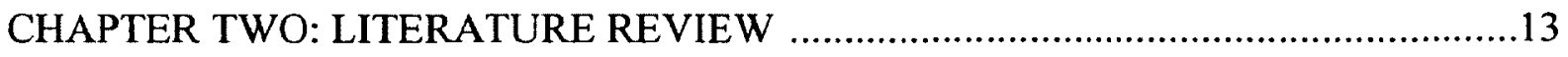

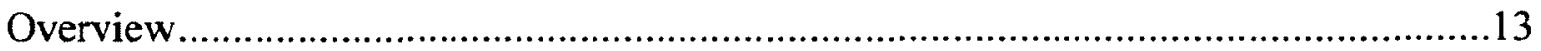

Chief Diversity Officers in Higher Education ...........................................................14

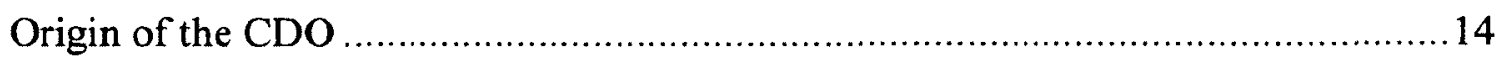

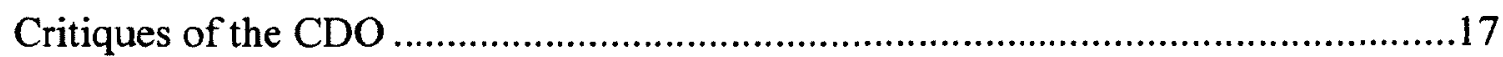

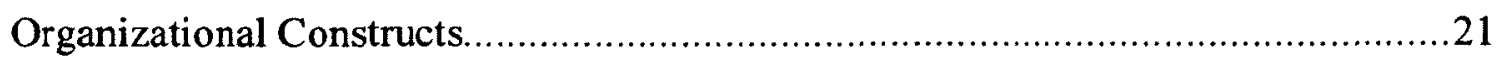

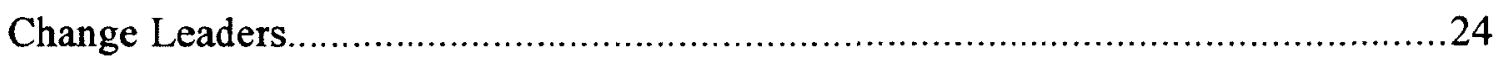

Organizational Change in Higher Education ...........................................................25

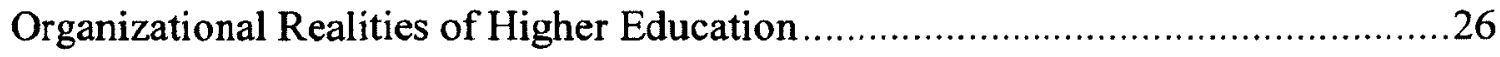

Positivist and Constructivist Approaches to Change .............................................30

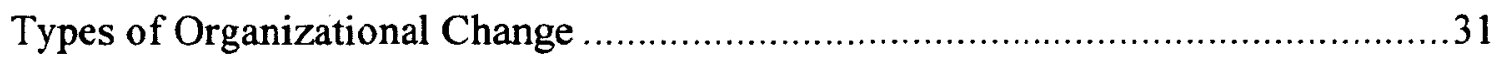

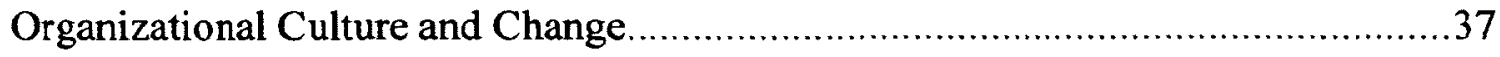

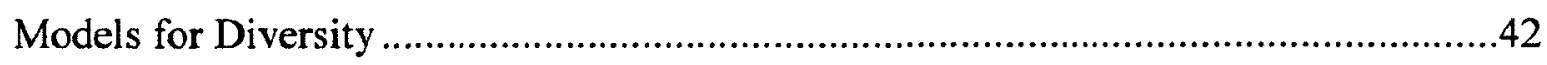

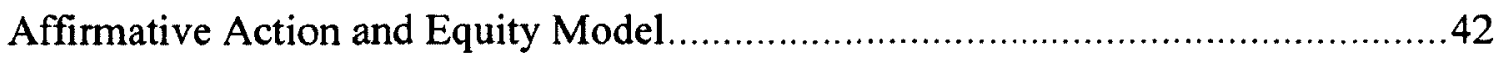

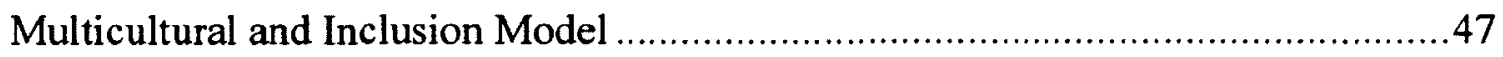

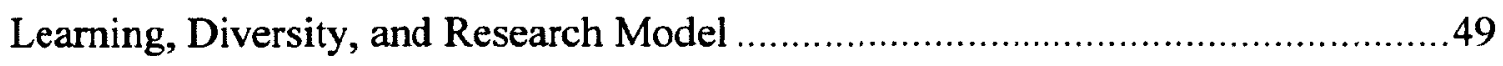

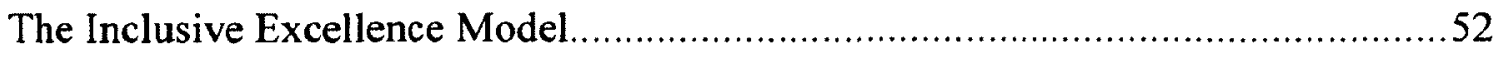




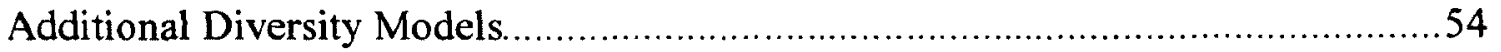

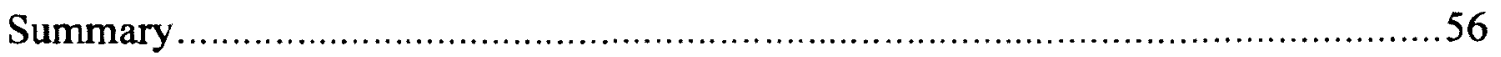

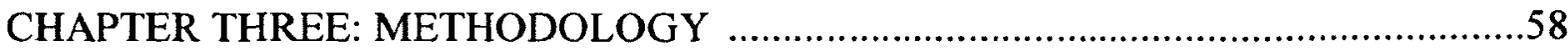

Methodological Overview ………….......................................................................5

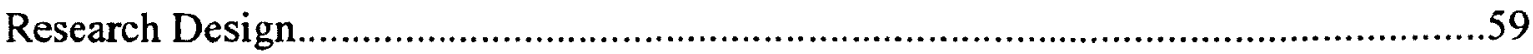

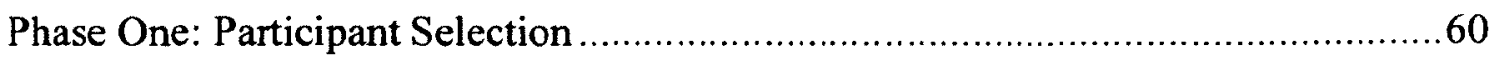

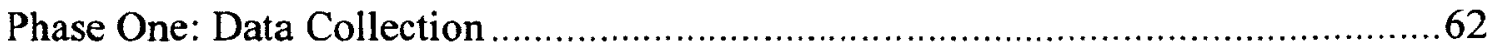

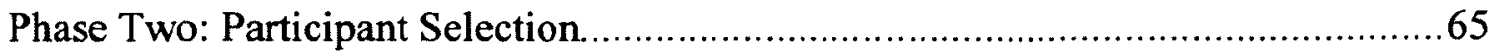

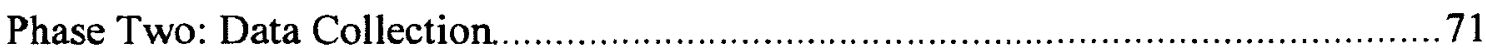

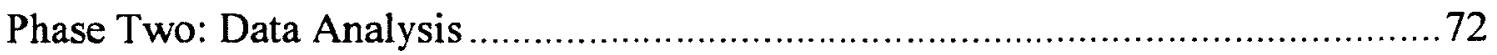

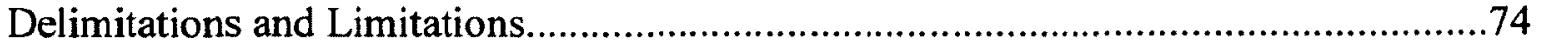

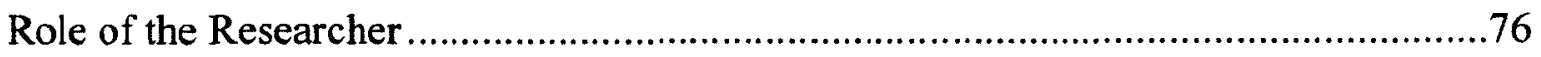

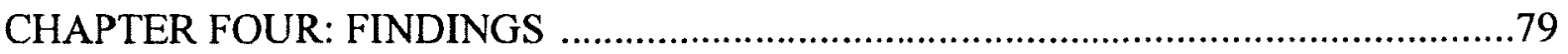

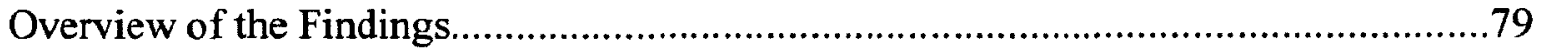

Review of the Purpose of the Study and Research Questions .......................................81

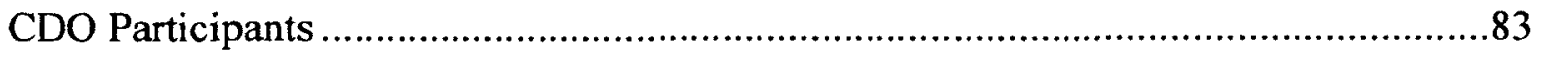

Role of the CDOs in Institutionalizing Diversity and Inclusion...................................89

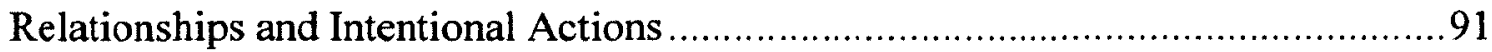

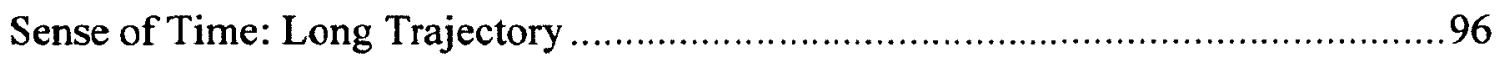

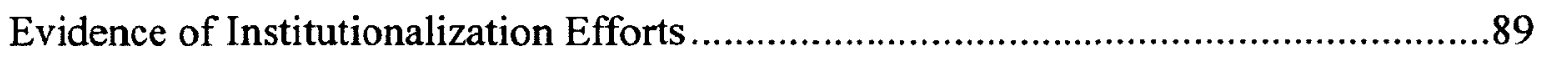

Broader Understanding of Diversity .............................................................. 100

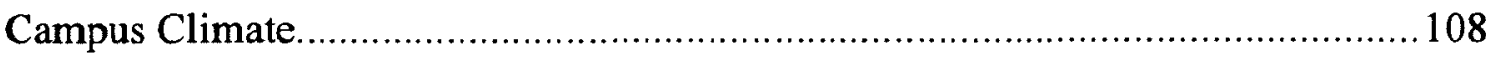

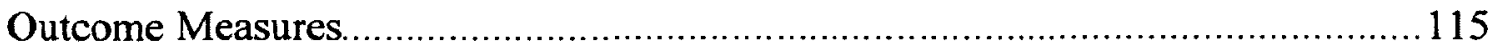

Key Strategies to Institutionalize Diversity and Inclusion .......................................118

Using Campus-wide Diversity Plans .............................................................119

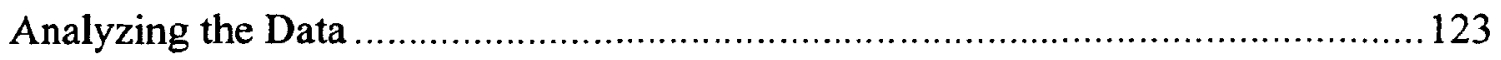

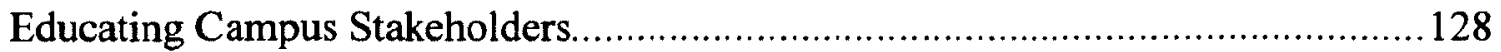

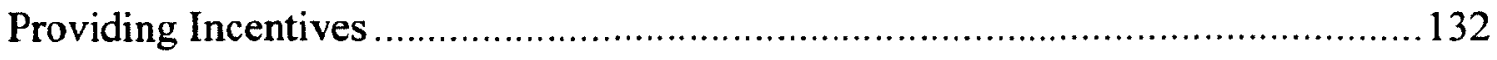

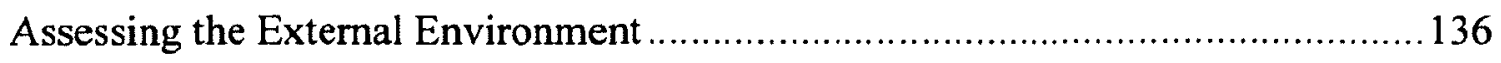

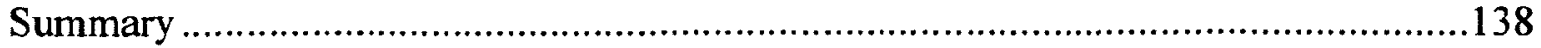


CHAPTER FIVE: DISCUSSION

Overview of the Chapter

Overview of the Purpose of the Study ....................................................................

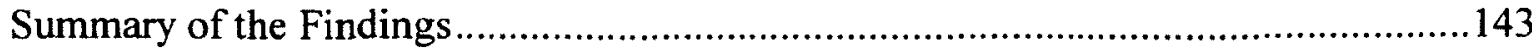

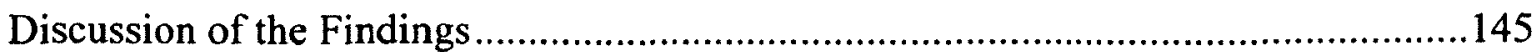

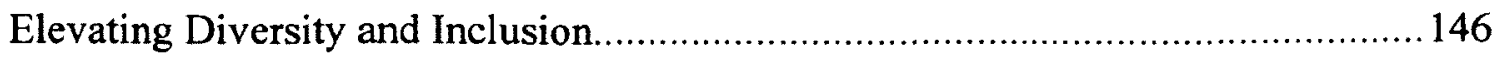

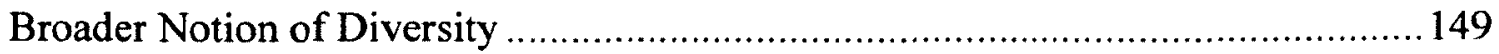

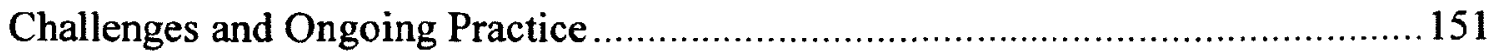

New Strategies for Institutionalizing Diversity and Inclusion...............................153

Recommendations for Institutional Practice ............................................................155

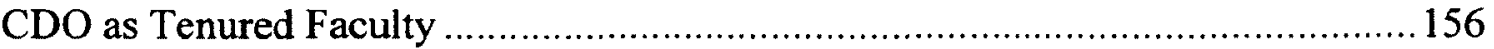

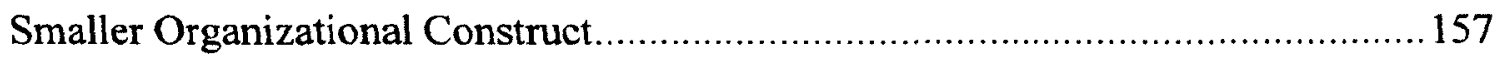

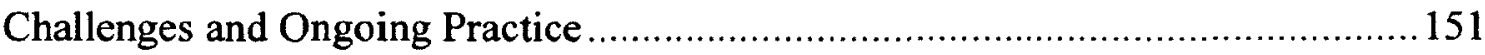

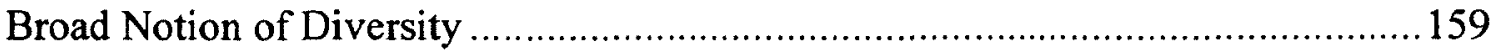

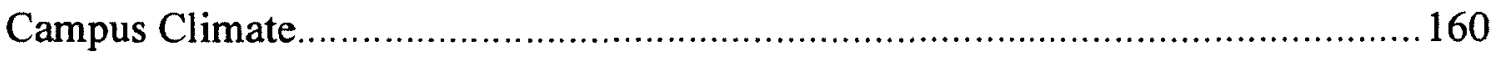

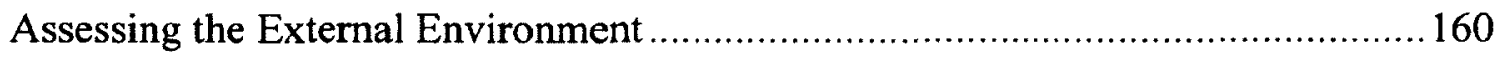

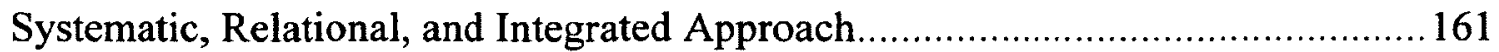

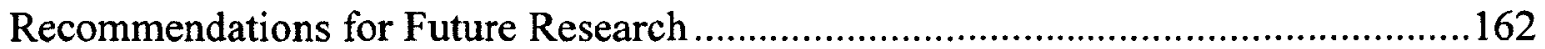

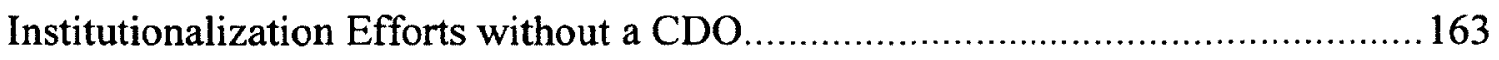

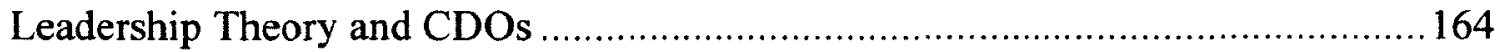

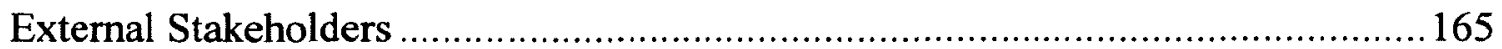

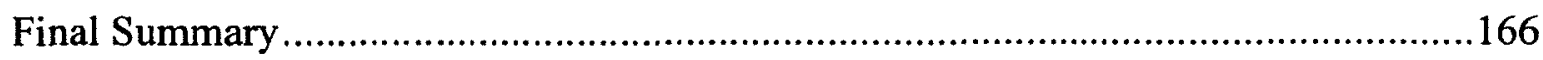

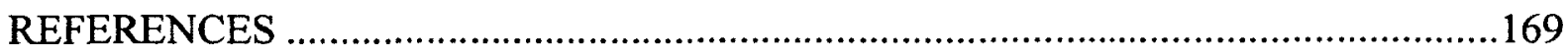

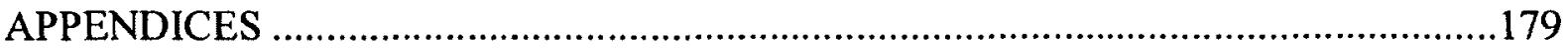

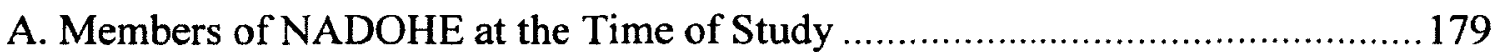

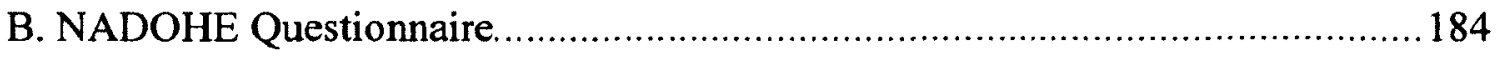

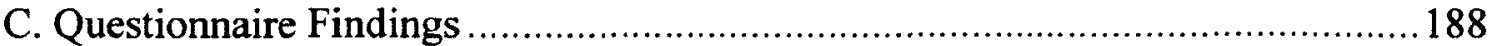

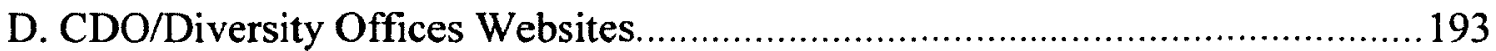

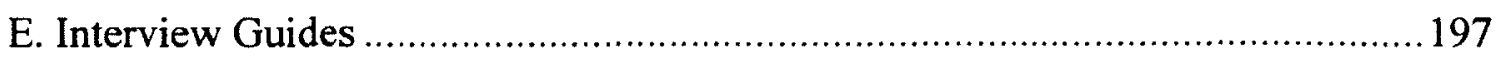

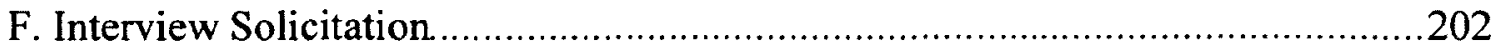

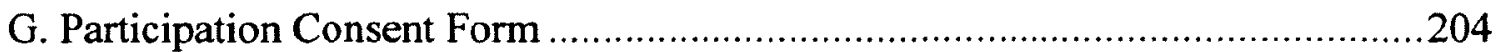




\section{LIST OF TABLES}

Table 1. Breakdown of Case Study Universities .....................................................68

Table 2. Breakdown of Study Participants ......................................................... 70

Table 3. CDO Reporting Line ..................................................................... 191

Table 4. Reason for Selecting Campus that Institutionalized Diversity or Close to

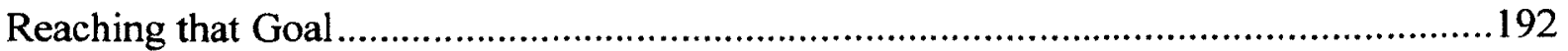




\section{LIST OF FIGURES}

Figure 1. Diagram of Research Design ........................................................60 


\section{CHAPTER ONE}

\section{OVERVIEW OF THE STUDY}

\section{Introduction}

Colleges and universities are susceptible to factors in the broader sociological environment that are impetuses for change (Birnbaum, 1989). Among these are shifting demographics, changes in the economy, new laws and state policies, and market place demands for a well-trained and globally minded workforce (Altbach, Gumport, \& Berdahl, 2011; Williams, 2013). These environmental factors are challenging how we examine diversity in this country. To illustrate this point consider these examples facing American higher education in 2014. In unprecedented numbers states and metropolitan cities in the United States are experiencing large growth in their minority populations. According to data collected by the Pew Research Hispanic Center, a record 40.4 million documented immigrants lived in the United States in 2011 (Mottel \& Patten, 2013). This is an increase of $30 \%$ from 2000 , when the total was 31.1 million. Many researchers and higher education professionals have asked how colleges and universities are adjusting to meet these new demands of racial and ethnic diversity? What about states that have traditionally not seen such a great influx of immigrants, i.e. Kentucky, South Carolina, Mississippi, Wisconsin, and North Carolina? What are they doing to address greater percentages of diversity in their educational systems?

Related, as Americans still struggle to recover from the 2008 worldwide economic recession, one has to consider socio-economic diversity. The recession in the

\footnotetext{
${ }^{1}$ According to Pew Research, five states have had a tremendous rise in immigrants since 2000 . Kentucky has experienced a growth of $97 \%$, followed by South Carolina with $88 \%$, Mississippi with $74 \%$, Wisconsin with $73 \%$, and North Carolina with $64 \%$.
} 
United States has resulted in a widening gap between economic classes, challenging who can afford university, the type of institution one will attend, when they will attend, and for how long. In the last five years, tuition and education costs have risen by $27 \%$ (College Board, College Pricing, 2012). These increases are occurring at the same time that federal grants are covering less of the total cost of education and family incomes across the entire economic spectrum have decreased by as much as $20 \%$ (College Board, College Pricing, 2012).

In addition, a new population of students is entering the educational landscape. Student veterans returning from combat in Iraq and Afghanistan are enrolling in community colleges and four-year universities with their own sets of challenges and needs. These students will require special support services that build community among fellow student veterans (DiRamio, Ackerman, \& Mitchell, 2008). A further shift in the population is Dreamer ${ }^{2}$ students; young children brought to the United States by their undocumented families. Annually 50,000 to 60,000 undocumented students graduate from American high schools (Passel, 2006). As Dreamers, they have received most of their elementary and high school education alongside students who are similar in every way except for residency status. Many wish to continue their post-secondary education but lack access to state and federal student aid to make their dreams reality.

These new factors are on top of the on-going challenges US higher education continues to face such as, disparities in enrollment and degree completion rates among historically underrepresented minority students (African-American, Chicano/Latino, and

${ }^{2}$ DREAMer is a term used to describe students who would benefit from the federal legislation proposal known as the Development, Relief, and Education for Alien Minors Act. 
Native American). The Latino college graduation rate continues to lag behind other groups, yet this population is rapidly increasing in the public K-12 school system (Santiago \& Calderón-Galdeano, 2014). Furthermore, greater consciousness is developing concerning lesbian, gay, bi-sexual, and transgender individuals pursuing higher education and their unique needs. These examples underscore transformations taking place in the United States that impacts American colleges and universities. How are colleges and universities addressing these new shifts and what is their process for managing change to meet new demands? Who is responsible for leading institutional change regarding diversity and inclusion?

The goal of institutionalizing diversity and inclusion so that all human experiences and identities are welcomed is commendable especially as research has proven the broad benefits of engaging and learning in diverse learning environments (Antonio, et al., 2004; Astin, 1993; Chang, 1999; Gurin, Dey, Hurtado, \& Gurin, 2002; Smith, 1997; Terenzini, Cabrera, Colbeck, Bjoklund, \& Parente, 2001). But exactly how universities achieve diverse, inclusive and equitable institutions is rarely studied. Research supporting diverse institutions and the need for inclusion and equity in higher education ask colleges and universities to undergo transformational change to produce globally and equity-minded college graduates (Chang, 2002; Hurtado, Milem, ClaytonPedersen, \& Allen, 1999). Yet, these requests alone are not sufficient for organizations to move into the next phase of drafting a diversity agenda to institutionalize diversity. Nor do they help with the decision to appoint a dedicated leader to lead the transformational change effort to be a diversity and equity minded institution. More research needs to be dedicated to studies of campuses that have successfully implemented a transformational 
change effort so that diversity and inclusion are prominent features of the culture and wider organization (Kezar, 2007).

\section{Problem Statement}

Colleges and universities are being affected by demographic shifts, political and economic policies, and demands to produce a culturally competent and well-trained globally minded workforce (Altbach, Gumport, \& Berdahl, 2011; Williams, 2013). To assist in these efforts, several colleges and universities have created a new senior level position to draft the campus' diversity agenda and to lead the campus in meeting its institutional goals of diversity and inclusion. This new position known universally in academia as the chief diversity officer (CDO) is the primary person responsible for leading the campus' diversity agenda and for institutionalizing diversity across the greater campus. Williams and Wade-Golden offer this definition of the CDO role:

Reporting to the president and/or provost, the CDO is an institution's highestranking diversity administrator. The position designates an individual who serves in a senior administrative role working toward diversity themed organizational change as a top priority at the highest levels of leadership and governance. The role is integrative, spans administrative and institutional boundaries and reflects the CDO's capacity to lead, coordinate, facilitate, enhance, and at times supervise the formal diversity capabilities of the institution in an effort to create an inclusive and academically rewarding environment for all (2013, p. 13).

Given this high-level executive leadership design, the functions and duties of the CDO are significant. A CDO has to lead a campus' diversity efforts and be the advocate and leader for sustained institutional change that supports diversity and inclusion.

Although newer to higher education, the CDO position has existed in the corporate world since the 1980 s, (Williams \& Wade-Golden, 2013). The CDO position is gaining popularity as universities look to well-trained individuals to lead strategic plans that institutionalize diversity and inclusion and transform the campus. As a result of this 
growth a professional organization known as the National Association of Diversity Officers in Higher Education (NADOHE) was formed in 2006. NADOHE supports CDOs in higher education and the goal of making institutions of higher education more diverse and inclusive. Formed out of a meeting of the American Council on Education's Center for Advancement of Racial and Ethnic Equity, NADOHE's stated vision is "to lead higher education toward inclusive excellence through institutional transformation" (NADOHE Vision Statement, 2014). It maintains a peer-reviewed journal, Diversity in Higher Education that publishes current research on diversity related issues facing the higher education enterprise.

Despite the rise of these new administrative positions and the addition of a professional organization to support the growing field, little empirical evidence exists about the role CDOs play in making campuses more diverse, inclusive and equitable places to study, live, work, and teach. This is problematic because as universities face pressures from the external environment to serve more diverse individuals and graduate culturally competent and globally-minded students, are they meeting the demand in a comprehensive and intentional manner. A CDO to lead institutional diversity and inclusion efforts may be one solution to obtaining a thorough and transformative change effort. However, more needs to be known about CDOs and their related role at leading institutional change toward diversity and inclusion before university leaders can confidently move in the direction of appointing a CDO at their institution. In particular universities with a CDO that have had success at institutionalizing diversity and inclusion need to be more widely studied in order to learn what strategies have contributed to their success and what role the CDO played in these efforts. If we know more about the role 
of CDOs at leading a transformational change effort in support of diversity and inclusion, then universities are better positioned to move in this direction.

\section{Purpose of the Study}

The purpose of this study is to understand how CDOs lead and/or contribute to the institutionalization of diversity and inclusion on their campuses so that they are positively accepted and normed principles by which the university operates and functions.

Specifically, this study aims to examine the role CDOs play in institutionalizing diversity and inclusion efforts that lead to transformative and sustainable change. In this study, institutionalization is defined as establishing a standard practice or custom in an organizational system (Curry, 1992; Kezar, 2007; Kramer, 2000) and is considered the final phase of a transformational change process toward a particular goal (Curry, 1992). A further purpose is to understand how the three campuses in the study, with the support of their CDO, have institutionalized diversity campus-wide so that these practices lead to a sustained institutional effort with measurable outcomes.

The role of $\mathrm{CDO}$ and the institutionalization of diversity in higher education are important aspects to study in tandem because they shed light on the actual versus perceived operationalization of diversity efforts in higher education. To institutionalize a practice, like diversity requires organizations to modify reward structures, policies, and the environment (Kezar, 2007). A CDO who is committed to leading a change effort that empowers others, holds people and administrative units accountable, and maintains a focused and integrated approach to the institutionalization of diversity and inclusion, helps institutions reach the final phase of a transformational change process. A successful institutionalization effort will elevate the role of diversity and inclusion and 
protect it even in times of economic difficulty or leadership uncertainty (Williams, 2013). Thus, this study aims to contribute new data to the field by examining exemplar colleges and universities that have a CDO and have excelled in efforts to institutionalize diversity and inclusion campus-wide. Additionally, this study adds to the literature and contribute promising practices to university leaders considering hiring a CDO and or looking to institutionalize diversity and inclusion on their respective campuses. Both are instrumental to advancing the practice of having more CDOs lead institutional efforts to institutionalize diversity and inclusion at their respective campuses.

\section{Research Questions}

Three research questions guided the development and design of this study:

$\mathrm{RQ \# 1.} \mathrm{What} \mathrm{role} \mathrm{do} \mathrm{CDOs} \mathrm{play} \mathrm{in} \mathrm{institutionalizing} \mathrm{diversity} \mathrm{and} \mathrm{inclusion} \mathrm{in}$ US universities?

RQ\#2. How do three exemplar universities know they have institutionalized diversity and inclusion? What are their outcome measures and who is involved in these efforts?

RQ\#3. What key strategies contribute to successful institutional efforts to sustain diversity and inclusion at three exemplar universities? What is the role of the CDO in these efforts?

\section{Methodological Overview}

A multiple case qualitative methodology was designed for this research study. Three case sites were identified after an electronic questionnaire was administered to members of the National Association of Diversity Officers in Higher Education (NADOHE). Only current CDOs were asked to complete the questionnaire. In addition, 
CDO participants were asked to identify two separate institutions of higher education that in their professional opinion had been successful at institutionalizing diversity at their campuses or were close to reaching that goal. A list of metrics developed from the literature defining necessary elements of a diverse and inclusive institution were provided to give some contextual parameters to the collected responses. As a result, thirty-nine unique institutions were identified, all four-year universities. A total of eight universities were identified multiple times by the respondents, which immediately moved these campuses up the list for consideration. From this shorter list, maximum variation sampling was used to identify three different universities with a CDO: two public large sized research universities and one medium sized private faith based institution. The campuses were also selected to achieve geographic diversity and included universities on the West Coast, Midwest, and the South regions of the United States.

Within the three case sites, a total of 16 qualitative interviews were conducted with various members of the university community engaged in campus' diversity-related efforts. Because this study involves the role of CDOs in campus institutional diversity efforts, the CDO was interviewed at all three case sites. The universities' Provosts with whom each of the CDO in the study had a close working relationship with or a reporting line with were also interviewed. From here, additional within case participants were identified using purposeful and snowball sampling. These interviews included faculty members, students (undergraduate and graduate), student affairs professionals, and other university administrators involved in strategic diversity efforts.

In preparation for the case site visits, time was spent researching and reviewing existing campus diversity efforts and the organizational structure of the CDO's division 
or purview via websites and other online materials. The interviews took place in person at each of the campus case sites, using an interview protocol developed for each participant type. A total of three days were spent at each campus interviewing individuals and observing the campus culture. Materials, brochures, and campus newspapers were collected and read to identify possible evidence of the campus' diversity-related efforts and to become further familiarize with the institution. These materials were utilized in the overall synthesis of the campuses' uniqueness and were used to inform the interviews as they were occurring at the case sites. A constant comparative approach was used to analyze the data during data collection and after.

\section{Significance of the Study}

This study focuses on the role of the CDO at three exemplar universities specifically known to be succeeding in their efforts to institutionalize diversity and inclusion campus-wide. Examining institutions that have experienced success, as determined by fellow CDO peers, offers more opportunity to learn how CDOs institutionalize diversity and inclusion. This study suggests that universities can institutionalize diversity and inclusion with the efforts of a CDO who is committed to leading a transformational change process that is supported by the institutionalization of diversity and inclusion. Specifically, change that moves diversity from the margins of the institution, where it is traditionally isolated and relegated to a minor role, to the center of the university were it is intentionally connected to the mission of the university and part of the university's collective consciousness.

Through intentional efforts that include relationship building, trust, and patience for a long change process, CDOs can help colleges and universities institutionalize 
diversity and inclusion so that it is a stable and permanent condition of the university that positively affects the way a university functions and makes decisions. These efforts lead by the CDO rely on strategies that bring campus stakeholders into the change process wherever they may be in their understanding of diversity, so that overtime diversity supporters and advocates increase and the diversity and inclusion effort has a greater opportunity to permeate the wider organization. In this sense, diversity becomes more than an annual exercise, task of compliance, or one person's job; rather, diversity and inclusion become part of a university's psyche upheld by a critical mass of supporters. In this study, the CDOs and their respective campuses under the guidance and direction of the CDO used various strategies to sustain their diversity efforts and to receive greater support and acceptance for diversity work. This study suggests that when campuses have a broader understanding of diversity that moves beyond traditional notions of race and affirmative action, the campuses are better poised to link the importance of diversity and inclusion to the mission of the university. This means that campuses have a more sophisticated and better understanding of diversity that exceeds traditional measures of counting the quantity of diverse people in the organization or solely focuses on factors related to student success of historically underrepresented groups. Furthermore, campus climate and the way campus community members feel at their institutions is an important aspect to study and understand in relation to diversity and inclusion, which each of the campuses in this study examined consistently. CDOs are likely to be better positioned to effect change by having a more accurate picture of what is taking place at their institutions and therefore can address what needs to be changed and sustained. 
Finally, this study revealed five specific strategies successful campuses with a $\mathrm{CDO}$ utilize to institutionalize diversity and inclusion. This is helpful to campuses considering hiring a CDO because they will need to identify ways to sustain their efforts and to build greater buy-in. Working without resources that can guide a CDO's efforts could be problematic and possibly lead to failed change efforts that are intended to support diversity and inclusion. By using the findings from this study, CDOs may be able to better help their campuses institutionalize diversity and inclusion in a more efficient and successful manner that will lead to transformational change.

\section{Definitions}

There are several commonly used terms throughout the dissertation, thus the definitions below may help clarify terms for the reader.

Chief Diversity Officer (CDO) - This term refers to the higher education executive level position responsible for setting and/or leading a campus' diversity agenda or strategic plan for diversity (Williams, 2013; Williams \& Wade-Golden, 2013).

Compositional Diversity - Refers to the number of minority and female faculty, staff, and students of a university. It is also commonly referred to as structural diversity whereby diversity can be quantified in terms of numbers of specific minority groups.

Diversity Agenda/ Plan - Is a concerted attempt by the campus to integrate diversity into the structure, culture, and fabric of the institution (Kezar, 2007).

Diversity-related Efforts - Pertains to the individual actions, offices, decisions, and policies that contribute to a campus' overall diversity goals at institutionalizing diversity and inclusion. 
Institutionalization - A process for establishing a standard practice or custom in an organizational system (Curry, 1992; Kezar, 2001; Kramer, 2000) resulting in a normative consensus about the intended change (Kezar, 2007).

Institutionalization of Diversity and Inclusion - Can be the outcome (Kezar, 2001) of the transformational change effort and the process (Curry, 1992) to achieve transformational change.

Transformational change - Refers to deep and pervasive change that has permeated the entire organization (Eckel \& Kezar, 2003) resulting in a paradigm shift (Kuhn, 1962;

Simsek \& Louis, 1994). 


\section{CHAPTER TWO}

\section{BACKGROUND LITERATURE}

\section{Overview}

The purpose of this study is to examine the role CDOs play in institutionalizing diversity and inclusion efforts that lead to transformative change. A further purpose is to understand how the three campuses in the study have institutionalized diversity campuswide so that diversity is a sustained institutional effort with measurable outcomes. It is important to look at the actions and behaviors of the exemplar universities and the role of CDOs in tandem because together they shed light on the actual versus perceived operationalization of diversity efforts in institutions of higher education. Before examining the data gathered at the three case study sites, a review of the existing literature is necessary. For purposes of this study, this literature review has been divided into three sections that frame the research problem.

The chapter begins with a review of the current literature on CDOs, which due to its nascent being is relatively small in comparison to other bodies of research exploring the significance and impact of diversity in higher education. Nonetheless, the origin of the $\mathrm{CDO}$ position, its critiques, organizational constructs, and vertical structuring is discussed, as well as its primary function as a driver of change. Because CDOs are hired to lead change efforts, the next section of the review delves into research on organizational change in higher education. In particular, the types of change operating in higher education is discussed, as well as the importance of factors such the organizational reality of higher education institutions, and the significance of organizational cultures operating within the academy that can assist or derail change efforts. The final section of 
the literature review examines various types of diversity models operating in higher education. These models are helpful to CDOs because they can help campuses define their diversity and inclusion goals and help operationalize their efforts. A detailed discussion of each follows. At the conclusion of this chapter, a brief summary of the sections and their connection to the problem statement and study is discussed.

\section{Chief Diversity Officers In Higher Education}

The design of this study focuses on the role CDOs play in institutionalizing diversity in US universities. Related, it examines how three exemplar universities with guidance and direction of their CDOs have institutionalized diversity and inclusion on their campuses. Next the study focuses on strategies the CDO and the case institutions use to institutionalize diversity and inclusion at their campuses so they are a sustained and permanent feature of the wider organization. In all three areas, the CDO is instrumental to the campus' diversity-related efforts. Understanding the history, impetus, challenges, and organizational structure of the CDO position before the other literature areas is important to the study due to the central role CDOs play in institutionalizing diversity and inclusion and the role they play in this study in particular.

\section{Origin of the $\mathrm{CDO}$}

Throughout the 1950s, 60s and 70s when access to higher education became more frequent to African-Americans and women, minority affairs offices were established to help students make the transition into male dominated white institutions (Williams, 2013; Williams \& Wade-Golden, 2006). As the civil rights movement gained momentum in the late 1960s, federal laws and policies created a need for compliance and accountability for institutions receiving federal aid. Then called minority affairs officers, equal opportunity 
officers, access officers or affirmative action officers, these early diversity officers were assigned the job of enhancing compositional diversity of the university's student body and faculty (Banjeri, 2005; Williams \& Clowney, 2007). The main goals of these efforts were to remediate and eliminate discrimination among federally protected identities (Williams \& Clowney, 2007). Many of these early positions and minority offices were relegated to the margins of the institution and were tasked with fixing the problem of low compositional diversity (Barceló, 2007). In addition to federal compliance obligations, these offices featured access programs geared toward "disadvantaged" communities, i.e., low socio-economic status and/or underrepresented minorities. Game changing federally supported access and retention programs brought about through the Economic Opportunity Act of 1964 and the Higher Education Act of 1965, introduced services such as Upward Bound, Talent Search, and Student Support Services, known collectively as TRIO (Office of Post Secondary Education, 2014). These programs were led by some of the first diversity officers. These offices function and importance to higher education then and now is critical; however, these early diversity officers lacked the institutional capital and authority to lead transformational change efforts on behalf of the university.

In contrast to the narrow focus of student access and institutional reporting obligations of the 1960s and 70s, today's CDOs have far-reaching levels of responsibility. Their area of focus now spans the entire campus community ranging from women and minority faculty hiring, student body compositional diversity, campus climate, diversity curriculum, international affairs, and disability services to name a few (Williams, 2013; Williams \& Wade-Golden, 2006). The most influential CDOs are "at the table" during discussions of institutional policy and core academic functions 
(Williams \& Wade-Golden, 2006). The highest-level CDOs are senior level administrative officers that report to the university president or chief academic officer, e.g., provost or executive vice president (Williams \& Wade-Golden, 2013). As Williams and Wade-Golden (2006) describe it, "where others work on issues of diversity as a matter of second or third priority, [CDOs] engage matters of diversity as a matter of firstpriority" (p. 2). Key to a CDO's ability to succeed is integrative and strategic relationships that allow them to work across several administrative functions in order to bring about campus-wide transformational change supportive of diversity and inclusion (Williams \& Wade-Golden, 2013).

A significant factor that distinguishes today's CDO from its mid to late $20^{\text {th }}$ century predecessors, is a wider and more inclusive definition of diversity (Williams, 2013; Williams \& Wade-Golden, 2013). Whereas earlier campus diversity officers' foci was on racial and gender diversity, mostly contextualized in a Black/White paradigm, today's CDO operates with a broader understanding of diversity that includes sexual identity, economic background, military status, religion, immigration status, age, ability, and more (Williams, 2013; Williams \& Wade-Golden, 2006). The institutional leveraging of these various identities can enhance the learning and development of students through intentional engagement and interactions, which in turn can make the conditions for learning greater (Williams \& Wade-Golden, 2006). This focus capitalizes on diversity as a resource rather than an act of obligation (Williams, 2013; Williams, Berger, McClendon, 2005).

Williams and Wade-Golden have conducted the largest study of CDOs in higher education. Their study involved over 100 interviews of individuals in higher education 
and a national quantitative survey sent to over 2,500 institutions with a diversity officer and received a 31 percent response rate. Based on these studies they have developed a grounded definition of the $\mathrm{CDO}$ role, which is the most cited in the short existence of the position. In addition to delineating the CDO's reporting structure and position within the university, Williams and Wade-Golden (2013) have synthesized the many functions and tasks of the $\mathrm{CDO}$ into this definition:

Working toward diversity-themed organizational change as a top priority at the highest levels of leadership and governance... [the $\mathrm{CDO}$ ] role is integrative, spans administrative and institutional boundaries, and reflects the CDO's capacity to lead, coordinate, facilitate, enhance, and at times supervise the formal diversity capabilities of the institution in an effort to create an inclusive and academically rewarding environment for all. Within this context, diversity is not merely a demographic goal but a strategic priority (emphasis added) that is fundamental to creating a dynamic educational and work environment that fulfills the teaching, learning, research, and service mission of the institution (p. 13).

Their research and subsequent crystallization of the role of CDOs has helped to legitimize the profession in the academy. It has also launched further studies, including this one, on CDOs and their complex role in higher education.

\section{Critiques of the CDO}

Despite the growing prevalence of CDOs in higher education, their position in academia is not without skeptics or opposition. These critiques can range from the perception of inauthentic intentions and superficial fixes, to beliefs that the campus is engaging in unfair practices and reverse discrimination. Opponents may question the amount of financial resources associated with CDO offices, while others may see efforts to appoint a lead person in charge of diversity-efforts as dodging the work of the president or provost. 
For example, some critics perceive the $\mathrm{CDO}$ position as a costly and symbolic gesture of pacification to angry protestors (Gose, 2013; Williams, 2013). In a 2011 opinion piece written by columnist Heather Mac Donald, the political author claims that while the University of California has had to endure consecutive cuts to it's budget, it has made fiscally irresponsible decisions in hiring CDOs with salaries exceeding $\$ 200,000$. She points out that while this has occurred, mid-level faculty salaries have remained stagnant and tuition has steadily increased. Mac Donald argues that CDOs are simply overpaid unnecessary "taxpayer-funded bureaucrats" and refers to their offices as diversity "fiefdoms." In her opinion university resources should be directed to academic programs and disciplines that advance science and industry and not toward diversity, which she considers to be narcissistic pursuits. While much of Mac Donald's article is hyperbolic in tone and rhetoric, her commentary is important to consider as one example of opposition. Constituents from within the university, including faculty and staff may also question the rationale for spending university resources on diversity initiatives, during times of fiscal constraint (Gose, 2013).

Williams and Wade-Golden (2008) affirm that CDOs can be a lightening-rod for criticism above any other top campus administrator. They indicate that critics range from those who simply oppose efforts at building more inclusive and equitable institutions to those who believe that hiring a $\mathrm{CDO}$ "removes the responsibility for diversity and inclusion from the university's president, faculty members, and the campus as a whole" (2008, p.1). Ben Gose (2013) draws our attention to a keynote address in 2008 by a faculty member from Brown University's Center for the Study of Race and Ethnicity in America. In the keynote's remarks, the noted scholar encouraged CDOs to walk-away 
from their jobs, as their presence has allowed presidents and provosts to pass on their failed responsibilities to the $\mathrm{CDO}$. Those, such as the faculty member calling for the walkout, believe that the appointment of a CDO removes accountability from those who are ultimately responsible for the campus' diversity and climate. Additionally, many fear that the appointment of a $\mathrm{CDO}$ implies that faculty and campus units no longer have to be concerned or bothered with diversity efforts because it is now the purview of the CDO (Williams, 2013).

Additionally, some critics believe the presence of a $\mathrm{CDO}$ on campus lowers the academic quality and prestige of the university (Williams \& Wade-Golden, 2008). These critics are fearful that faculty and other campus leaders will cave to political pressures from the $\mathrm{CDO}$ to increase diversity, which they believe will result in lowering academic standards. Some of these critics claim that CDOs and their supporters sanction "reverse discrimination" against majority groups such as, Caucasians and men (Clark, FaschingVarner, \& Brimhall Vargas, 2012; Williams \& Wade-Golden, 2013). Unfortunately, some of these critiques come from within the organization intent on preserving the "old way" of doing business. In a collection of case studies highlighting the challenges of CDOs, editors Christine Clark, Kenneth J. Fasching-Varner and Mark Brimhall-Vargas (2012) note that CDOs consistently face their greatest challenges from internal deficiencies that result in unsupportive campus cultures.

Other examples of critiques come from leaders and members of the campus community who believe that the $\mathrm{CDO}$ is not the scapegoat, but rather the savior, which can lead to disappointment from unrealistic expectations. Williams and Wade-Golden (2013) refer to this characterization of a CDO as "diversity messiah." In these situations 
CDOs are inaccurately thought of as the "silver bullet" to fixing the campus' diversity problems. An example of where this can occur is in the aftermath of a diversity crisis. A diversity crisis is an incident that draws unwanted public attention to the university's unwelcoming campus climate (Williams, 2013). These types of crises can take the form of a racially themed party, sexual assault, or derogatory remarks by a university official (Williams, 2013). After a diversity crisis pressure from students, faculty, alumni and/or external community members is likely to occur. In these situations, constituents will often demand the university take corrective action. As a result, a CDO is often appointed or hired as the fix to the campus' diversity problems (Barceló, 2007; Williams, 2008).

However, as Williams (2008) points out these seemingly positive corrective steps can potentially cause greater harm to the campus and the $\mathrm{CDO}$ if the campus is not willing to change its underlying beliefs and assumptions regarding diversity and inclusion. After the incident unfolds and protest and demand occur, the university can take control of the incident and make change by publically supporting diversity and commissioning a planning group to devise a diversity agenda for the campus (Williams \& Clowney, 2007). The planning effort should include deliberating and discussing the diversity agenda widely with campus stakeholders and community constituents (Williams \& Clowney, 2007). From here, Williams and Clowney (2007) recommend the campus make another public commitment to fully implement the plan immediately. This includes allocating the appropriate financial and staff resources to support the plan. If there is a delay in the implementation and a lack of resources to carry out the plan; then the change effort is at best superficial (Williams \& Clowney, 2007) and criticism and more unrest are likely to follow. Despite a CDO's best effort to bring transformation to the campus 
after a difficult situation, without the support of the campus' senior leadership through resources and public backing, the CDO may be seen as an overpaid bureaucratic who could not solve the diversity problem.

There are a number of positive outcomes a successful $C D O$ in a supportive environment can accomplish; however, the position is not without controversy or critique from both internal and external groups. As these challenges are known and dealt with early on, the onboarding and transition of a CDO into the university might be smoother for the campus.

\section{Organizational Constructs}

The organizational construct of the $\mathrm{CDO}$ position is important to understand because it explains how the CDO functions within the university (Leon, 2014). Each $\mathrm{CDO}$ position has a vertical structuring that positions the $\mathrm{CDO}$ and their office within a preexisting hierarchy inside the university. The vertical structuring of the CDO office is related to the diversity structure of the campus that includes the institution's definition of diversity, senior leadership support for diversity, as well as planning systems and accountability structures for diversity (Williams, 2013).

Williams and Wade-Golden (2103) suggest that more than any other aspect of the CDO position, the vertical structuring is the most critical and at times can be contentious. Failure to enact the right structure for a university can result in superficial changes versus actual leadership capability. In their research of 104 CDOs and their respective offices, Williams and Wade-Golden (2013) identified three common CDO organizational constructs, which they refer to as archetype structures. They are: the Collaborative Office Model, Unit-Based Model, and the Portfolio Divisional Model. Because 
universities are dynamic organizations influenced by internal and external factors, the CDO archetypes can change and fluctuate with the introduction of new variables, such as a new university president, budget cuts, or a crisis that requires swift action. A brief overview of each organizational construct follows.

Collaborative office model. In the Collaborative Office Model human resources are limited with the exception of administrative support and or student staff support. These CDO positions are often "one-person shops" (Williams \& Wade-Golden, 2013). As a result, "nearly all projects and initiatives are implemented through collaborative relationships and lateral coordination" (p. 166). In the absence of a larger staff, the CDO has a "high-ranking title, personal leadership, and the ability to negotiate with limited financial resources" (p. 168). Strengths of this model include limited disruption to the current campus organizational structure and low costs due to a relatively small office size. Also during periods of restructuring or other organizational changes, this model provides greater flexibility and ability to redefine the role of the CDO. Despite this smaller structure, the Collaborative Model does provide a symbolic expression of commitment to diversity; however, this can often be construed as too symbolic versus having a larger showing of "material commitment to the CDO role design" (p. 168). It also can create unequal footing between the $\mathrm{CDO}$ and comparable senior administrative roles that have larger offices and staff. Williams and Wade-Golden's research found that $40 \%$ of CDOs reported working in a Collaborative Model and found that many of these CDOs conveyed being stretched too thin because of understaffing and numerous requests to participate in initiatives, committees, position searches, etc. (p. 169). 
Unit-based model. The second archetype structure identified by Williams and Wade-Golden (2013) is the Unit-Based Model. In the Unit-Based archetype "a premium is placed on lateral coordination and relationship building" but is also "characterized by a more robust vertical capability" (Williams \& Wade-Golden, 2013, p.173). Thirty-one percent of CDOs reported working in this model, which has a larger reporting staff than the Collaborative Model. According to Williams and Wade-Golden's research these individuals can include lower-ranking diversity officers as well as data analysts. These offices are poised for more capability to create new diversity initiatives, projects, and events as well as "engage in collaborative relationships with others to seed new possibilities" ( $p$ 168). The downside to this model is the CDO office may have potential organizational conflict with diversity offices that do not fall under the CDO's control. Additionally, this archetype is also likely to be seen as a "more cost intensive model" due to the larger staff and organizational responsibility of events and initiatives tied to the CDO (p. 169).

Portfolio divisional model. The last archetype defined by Williams and WadeGolden's (2013) research is the Portfolio Divisional archetype. Twenty-eight percent of the CDOs they interviewed reported being in this model, which they contend is the most vertically situated with defined top-down chains-of-command. The model incorporates aspects of the previous two models but has a larger organizational structure, which may include other senior diversity officers that oversee units within the CDO's portfolio. The researchers found that of the CDOs in this model, the majority held titles such as vice president, vice provost or vice chancellor rank and were commonly found at institutions with over 10,000 undergraduates. They also identified 10 units that could potentially fall 
under the direct supervision of the $\mathrm{CDO}$ in the Portfolio Divisional Model. They are: minority and cultural affairs; cultural centers; ethnic and gender studies; retention and pipeline initiatives; community outreach; affirmative action and equity; training and development; general administration student services, such as admissions and registrar, research centers and institutes; and international affairs. Strengths of this model include an enhanced capability "to create new diversity deliverables in terms of new initiatives, projects, and events" as well as the capacity "to leverage the current diversity infrastructure" (p. 169). Williams and Wade-Golden suggest that this model sends a "powerful symbolic message of commitment to the campus diversity agenda" and "mirrors the divisional structure of comparably titled roles" (p. 169).

The research conducted by Williams and Wade-Golden provides a basis and current understanding for present day CDO models. All three CDOs in this study used the Williams and Wade-Golden model language to describe their offices' organizational structures. Two of the case sites identified their offices as operating within the Collaborative Model, while one described the CDO office at their campus to work more within the Portfolio Divisional Model. Despite the variance in their organizational constructs and vertical structuring, all three CDOs in the study remained effective at leading change.

\section{Change Leaders}

The primary function of a CDO is to lead a change effort that is supportive of diversity and inclusion (Williams \& Wade-Golden, 2006). According Williams and Wade-Golden, "[CDOs] are best defined as change management specialists because of the importance that they place on strategies designed to intentionally move the culture of 
their institutions" in support of diversity and inclusion (p.4). They go on to state, "at least at the surface, no CDO is hired to maintain the status quo" (p.4). As a result, when an institution considers hiring a CDO it is essential that they look for a candidate that "posses an outstanding command of the elements and dynamics of organizational change" (p. 5). Additionally, given the inherent "politicized process" of leading a change agenda, "they must [also] have commitment to see the change process through its challenges and rough spaces to effect deep structural change" (p. 5).

As change leaders, Williams and Wade-Golden (2007) identified eight core areas CDOs do to lead a change effort supportive of diversity and inclusion. They are: (1) elevating diversity; (2) leading strategic planning; (3) building diversity infrastructure; (4) enhancing structural diversity; (5) informing the search process; (6) cultivating diversity awareness and appreciation; (7) developing institutional diversity accountability systems; and (8) integrating diversity and academics. As they discuss, the role of the CDO is no small task. The overarching function of the CDO is to create lasting change toward diversity in the organizational environment. This is not simply about listing all the campus' diversity efforts and programs to show what the university is doing (Smith, 2009), but rather is intentional and concerted effort to drive change throughout the entire organization. As change leaders, the work of the CDO includes linking decentralized programs, services, and initiatives, identifying areas for implementation of diversity programs, and holding departments and units accountable (Barceló, 2007; Williams \& Wade-Golden, 2103). How the CDOs in this study accomplished this will be discussed in detail in Chapter 4.

\section{Organizational Change in Higher Education}


Research and theories discussed in the previous sections contend that CDOs are change management specialists specifically hired to lead change that is supportive of diversity and inclusion. A simple definition of change describes it as an alteration in the structure, processes, and/or behaviors of a system (Zaltman \& Duncan, 1977). This section of the review begins with a discussion on the organizational realities of higher education, which is unique and can make leading a change effort toward diversity and inclusion difficult for a CDO. Second, this review discusses various types of change efforts conducive to supporting diversity and inclusion. Finally, this section of the review concludes with a discussion on various types of cultures operating within higher education. A change effort lead by the CDO would have to take into account its own institutional culture.

\section{Organizational Realities of Higher Education}

Before discussing types of change efforts in higher education, it is important to first understand the organizational reality of higher education, which is an enterprise filled with smaller sub-organizations and multiple cultures. To the average outsider it may appear that universities are purely hierarchical organizations and therefore change should be easy to enact using a top-down approach. For example, there is a president; a cabinet comprised of vice presidents and deans, and under each of those vice presidents and deans are various offices and units. However, there is more complexity involved that a CDO should be conscious of before commencing a change effort. Organizational ambiguity, loose coupling, and cybernetic systems are a few examples of the organizational realities operating within higher education. 
Organizational ambiguity. First, universities can be very ambiguous organizations to engage in change efforts. Universities often have unclear and contested goal structures (Baldrige, 1980). Victor Baldridge (1980) describes this as "almost anything can be justified, but almost anything can be attacked as illegitimate" (p. 43). An outsider unfamiliar with how a university is organized might ask, who is in control, where does power and authority lay, who sets organizational goals, how are units held accountable and by whom, and how is systematic change even possible in such a complex organization with multiple identities and leaders? These types of questions address the organized anarchy often found in higher education (Baldridge, 1980; Cohen \& March 1974).

In their classic work Leadership and Ambiguity (1974), Cohen and March described universities as organized anarchies because they have problematic goals, unclear technologies for solving problems, and fluid decision-making processes with inconsistent participants. Decisions of an organized anarchy "are a consequence produced by the system, but intended by no one and decisively controlled by no one" ( $p$. 34). This can be particularly harmful to diversity efforts if there is no clear leader. What some may find surprising is university presidents can have some of the most ambiguous roles in the university (Cohen \& March, 1974), which can be disconcerting to a CDO who is seeking support from institutional leaders to lead a change effort in support of diversity and inclusion. Cohen and March have identified four ambiguities in a university president's role that is likely to affect a $\mathrm{CDO}$ in their work. The first ambiguity is of purpose describes vague and unclear goals university presidents often make, such as "diversity is important." Unclear goals can be in potential conflict with 
other stated goals or might be seen as an unworthy effort by other campus stakeholders (Cohen \& March, 1974). The second ambiguity that may affect a CDO and the campus' diversity agenda is the ambiguity of power, which addresses the illusion of power and authority a president has to make meaningful change. An example of this is a public university president in a state that is prohibited from using practices of affirmative action. The president may be facing pressures to make the campus more diverse and inclusive, but he or she may not have the full capability to fulfill the change to the satisfaction of those demanding action. In addition, the president may have less power and authority than the faculty senate body or board of trustees (Cohen \& March, 1974). These organizational ambiguities are important to consider as the CDO attempts to lead change.

Loose coupling and cybernetics. In addition to organizational ambiguity, another characteristic of universities that can impact change are its loosely coupled systems. Loose coupling allows for separate entities to exist in an organization and to be affiliated or associated with one another, but are not exclusively dependent on one another (Weick, 1978). "Loose coupling lowers the probability that the organization will have to - or be able to - respond to each little change in the environment that occurs" (Weick, 1978, p. 21).

Loose coupling has been a significant feature of universities ability to evolve (Weick, 1978). If all of the elements in a university are loosely affiliated, then any one unit can adjust and modify it without disrupting the entire system (Weick, 1978). A benefit is that change can be swift and economical because it is not reliant on an overly bureaucratic process commonly affiliated with a tightly coupled and regulated system. Additionally, loosely coupled organizations allow for greater autonomy and self- 
determination within the parts of the organization (Weick, 1978). This feature is highly coveted by faculty who value the ability to assert their own identity and culture associated with their academic disciplines and or departments. However, while loose coupling allows for adaptations to occur at a local level, loose coupling can make it difficult to implement transformational change. This is because the message and vision for change has to be absorbed into each individual department and unit. In this case, reaching total agreement for the change may be difficult and a lengthy process. Some argue universities are reluctant to any type of change because it disrupts the autonomous nature of well-established departments entrenched in their own cultural and behavioral norms (Williams, 2013). Furthermore, department members may see their academic interests in conflict with the change effort being lead by university administrators (Birnbaum, 1988).

How a university responds to change is often described using the metaphor of feedback loops. Robert Bimbaum (1989) developed the cybernetic paradigm for university structure and functioning. He suggests "organization control systems can be described in terms of sensing mechanism and negative feedback loops that collectively monitor changes for acceptable levels of functioning that activate forces that return institutions to their previous stable state," or homeostasis (p. 238). According to Birnbaum (1989), this model is the only explanation for regulating a complex, loose coupled, organized anarchy that appears to be operating from several different organizational frames.

A drawback of the cybernetic model is that it supports the belief that universities are not inclined to deep fundamental change, as they are more comfortable with 
addressing change that preserves the status quo or returns the organization to homeostasis levels (Bess and Dee, 2008). Another downside is that the cybernetic organization only recognizes feedback that is familiar to the organization (Birnbaum, 1989). For example, if an institution has never addressed diversity or inclusion on its campus it may not recognize feedback it is receiving related to diversity. In this situation, a university can potentially overlook important issues if the feedback loops are not sensitized to pick them up. If the system does not recognize the feedback loop then it cannot address the need for change. However, if an organization does pick up on the feedback, the underlying question becomes, is the organization learning from the feedback by making intelligent decisions that will aid the organization long-term or is it merely making a correction in order to stabilize the organization to it's present form (Argyris \& Schon, 1978). The presence of a CDO could help with making sure the feedback was used to improve the organization and making lasting change.

\section{Positivist and Constructivist Approaches to Change}

Attending to the organizational environment and unique qualities of colleges and universities provides the opportunity for a richer understanding of the types of change possible in higher education. There are two ways in which change in higher education can be examined (Bess \& Dee, 2008). The first is the positivist approach, which helps institutional leaders define organizational change through clear organizational constructs. Bess and Dee (2008) argue that most institutional leaders responsible for leading a change effort often utilize positivists constructed models that provide clear steps and definitions. Scholars Kezar and Eckel $(2002,2004)$ use positivist approaches to help institutional leaders define organizational change. In their research, organizational 
change relies on teleological models, which tend to be linear, planned, and contain clear stages for implementation (Kezar, 2001). Their research is directed at the individuals responsible for devising the change model and leading the effort, e.g., presidents, governing boards, and even CDOs.

The second way to view change in higher education is through a social constructivist orientation that utilizes multiple interpretations of reality that exist within the organization (Bess \& Dee, 2008). Paul Trowler (2008), William Tierney (1988, 1991), and George Lueddeke (1999) have studied organizational cultures in higher education and have used this perspective to understand the success of change efforts. The lens in which they view organizational change is not nearly as linear or predictable as the teleological model. A social constructivist approach requires an in-depth look at the many sub-units within the organization and their view of reality, which requires an indepth analysis of existing patterns of behavior (Bess \& Dee, 2008). Although time consuming, some argue constructivist approaches that dive deep into the organizational culture are the most successful at achieving enduring change (Trowler, 2008; Lueddeke, 1999).

\section{Types of Organizational Change}

Moving from the ways in which change can be approached (positivist or constructivist) to various types of institutional change, this next discussion can help a CDO select a change process that is appropriate to the desired goals of the change effort. Without a proper understanding of the various types of change efforts confusion can ensue, which may result in a failed change effort (Kotter, 1996). Two of the most 
prevalent change types in higher education are adaptive change and transformational change.

Adaptive change. Adaptive change is associated with an evolutionary change model (Kezar, 2001). In these instances, universities are faced with growing pressures from the external environment to adapt to new demands (Kezar, 2001). If universities do not adapt to meet new demands, they are likely to fail. Economists Clayton Christensen and Henry Eyring (2011) argues higher education, has adapted to new demands entering the educational market place for decades. In a term that he refers to as "disruptive innovation," the authors theorize that the higher education system has been disrupted several times with innovations not as strong as the original, but more enticing due to greater affordability and accessibility. Two examples are community colleges as an alternative and more affordable pathway to a four-year degree and online universities, which may not be of the same caliber of traditional universities but do accommodate a wider pool of prospective students (Christensen \& Eyring, 2011). In both instances most traditional four-year universities adjusted and eventually adapted to these new alternatives in the educational marketplace.

Two empirical studies on higher education organizational change conducted by Sporn (1999) and Lueddeke (1999) also support adaptation theories. Both scholars posit that social, political, economic, and technological factors from the external environment cause universities to adapt in order to meet the demands of the outside system, which is similar to Birnbaum's (1989) cybernetic model.

Sporn's qualitative case study research on six universities in the United States and Europe identified seven conditions needed for adaptive change to occur. The first stage 
is prompted when adaptation is triggered by environmental demands, which can be defined as crisis or opportunity. Second in order to adapt, universities need to develop clear mission statements and goals. Third, an entrepreneurial culture in the university enhances the adaptive capacity of the organization. Next, she points out that a differentiated structure enhances adaptation. Fifth she acknowledges that a professionalized management helps with the adaptation. Sixth, shared governance is necessary to implement strategies of adaptation in order to seek broader support and buyin. And finally, committed leadership is an essential element for successful adaptation.

Lueddeke's (1999) research extends the notion of adaptation into a model that embraces a generative learning organization. Influenced by Peter Senge's (1990) work on learning organizations, Lueddeke argues generative organizations are more creative at solving institutional problems. As a result, learning organizations are better able to meet the internal needs of the organization, which makes the university more skilled at managing complex change. Based on his research, Lueddeke developed, the AdaptiveGenerative Developmental Model or A-GDM, which consists of six components. According to Lueddeke, in order to change from being adaptive to generative, institutions need to first routinely conduct needs analysis of the organization. Second, they need to devote resources to research and development to be able to implement new strategies. Third, a clear plan and an agenda for strategy formation and development are necessary. Fourth, institutional resources need to be allocated to support the generative learning environment. Along the lines of strategy formation and development, there needs to be a clear plan for implementation and dissemination throughout the organization. And sixth, 
there needs to be ongoing evaluation of these creative solutions to make sure the change effort is meeting the need the organization identified in stage one.

In summary, adaptive change addresses demands coming from the external environment, which can include trends impacting diversity and inclusion. However, adaptive change tends to be more reactive rather than proactive. For institutions seeking a more proactive and therefore controlled change effort, transformational change is likely to be a better option, particular for CDOs responsible for being the leaders of the change effort.

Transformational change. Universities desiring significant change in the environment might envision transformational or radical change for their institution. In transformational change the old skin or identity of the institution is shed and a new identity emerges (Greenwood \& Hinings, 1996). Complete transformational change is often difficult to achieve and requires the institution to undergo a paradigm shift (Kuhn, 1962; Simsek \& Louis, 1994). Documented paradigm shifts and the efforts to sustain them are infrequent in the literature (Boyce, 2003; Clark, 1983; Simsek \& Louis, 1994) because most often, universities seeking transformational change tend to hold onto the old paradigm and blend it with the new (Simsek \& Louis, 1994). A failure to achieve a paradigm shift weakens the transformational change effort (Simsek \& Louis, 1994). In these situations, universities are typically engaging in surface level changes only, which might be effective for short-term diversity goals but does not address long-term change efforts that need to go deeper within the organization (Williams, 2013). Surface level changes can also be described as first-order changes that are incremental, developmental, evolutionary, and linear (Boyce, 2003). First-order change efforts tend to be easier to 
track and can be measured easily. As such campus leaders can feel a sense of accomplishment when they meet a target that is associated with a first-order change and yet, they may not be fully addressing the more complex change that is needed to achieve a paradigm shift.

Despite being more difficult to attain, transformational change is most commonly associated with diversity and inclusion work because it involves a more comprehensive change effort that involves the complete organizational system and its constituents. The most recent empirical studies on transformational change in higher education are from Adriana Kezar and Peter Eckel. Both scholars have been highly active in studying the phenomena of leadership and it's influence on organizational change in higher education. According to the scholars, transformational change affects institutional cultures by being deep and pervasive, intentional, and occurring over time (2003). To illustrate the difference between transformational change and other types of change, Eckel and Kezar developed a model of change that contrasts transformational change against other types of change (2003). For them, there is change that is an adjustment (neither deep nor pervasive), change that is isolated (may be deep but not pervasive), and far reaching (maybe pervasive but not deep). In their model, only transformational change is both deep and pervasive.

In 2002, Eckel and Kezar conducted a five and a half year study on six universities that had shown the most transformational change. Their research found that transformational change relies on how the university makes sense of the transformation process. Eckel and Kezar's research identified five core strategies that universities showing progress at reaching transformational change possessed. They are: senior 
administrative support, collaborative leadership, robust design, staff development, and visible action. According to Kezar and Eckel (2002), these five strategies "helped individuals conceptualize a new identity, feel worthwhile about their efforts, and were brought along with the institutional agenda" (p. 303).

Institutionalization. To obtain transformational change the institutionalization of the desired state or condition has to occur (Kezar, 2007). For purposes of this study, diversity and inclusion have to be institutionalized for transformational change to occur. Institutionalization can be used to describe a process and an outcome (Kezar, 2001). As a process, institutionalization consists of three phases (1) mobilization, whereby the system is prepared for change, (2) implementation, whereby the change is introduced into the system, and (3) institutionalization, whereby the system becomes stable in its changed state (Curry, 1992). In the last stage, institutionalization is the outcome of the change process.

Institutionalization is also defined as a standard practice or custom in a human system (Curry, 1992; Kramer, 2000). Characteristics of an institutionalized practice include: routine, widespread, legitimized support, permanent, and resilient (Kramer, 2000). Kezar (2007) suggests, "leaders who are committed to creating more inclusive campus environments are committed to institutionalizing change" (p. 415). They are committed to a new way of doing work that supports diversity and overtime becomes routine and standard feature of the organization. To institutionalize a practice, such as diversity and inclusion, "requires organizations to modify reward structures, polices, and the environment" (Kezar, 2007, p. 415). Over the course of the institutionalization, capacity is built, support is cultivated, and the system integration [in this case diversity 
and inclusion] is facilitated (Kezar, 2007). As a result of efforts to institutionalize diversity and inclusion, changes may occur to policies, practices, structures, and the climate/culture of the organization (Kezar, 2007). In this study, how CDOs institutionalize diversity and inclusion to be a more permanent feature of the organization was examined.

\section{Organizational Culture and Change}

Institutional leaders within higher education are more likely to achieve change by thoroughly knowing the institutional culture before attempting to carry out the change process (Bensimon, Neumann, \& Birnbaum, 1983; Birnbaum, 1988; Bolman \& Deal, 1997; Eckel \&Kezar, 2002; Tierney 1988). In fact throughout their research, Eckel and Kezar emphasize the importance of culture, arguing that attending to culture is what the transformational process is all about; it is the process that changes culture in a specified direction. Beyond culture, the work of transformational change has to get at the underlying assumptions of the organization, which make up the innermost core of institutional culture (Eckel \& Kezar, 2003). These underlying assumptions tend to be revealed when they conflict with espoused beliefs of the organization (Argyris, 1994; Schein, 1992).

For CDOs and other institutional leaders, culture can be studied in two ways; through the nomothetic and idiographic approaches (Bess \& Dee, 2008). A nomothetic approach to organizational culture attempts to categorize types of cultures in generalized terms that provide a typology in which to slot specific examples of culture (Trowler, 2008). A nomothetic framework of culture is helpful to universities because it provides a starting point from which universities can recognize their own existing culture(s). An 
idiographic approach on the other hand, examines the unstable and individual aspects of a university to determine its unique and overarching culture (Trowler, 2008). This type of analysis results in a unique culture that is not generalizable and does not fit into any preexisting typology (Trowler, 2008). Both approaches are discussed below.

Nomothetic approach. A nomothetic approach to understanding culture is helpful to a CDO who needs to quickly comprehend possible cultures operating in the organization. The benefit of nomothetic approach to a CDO is there is an existing typology that helps identify the culture at hand. For example, William Bergquist's research identified four cultural frameworks operating in higher education; the collegial culture, managerial culture, developmental culture, and advocacy culture (1992). The collegial culture values the role of the faculty and the "quasi-political" governance faculty hold within the institution. The managerial culture values the organization of the university to meet goals established by the university. According to Bergquist (1992), universities with this culture have high standards for meeting institutional goals and utilize institutional data to measure progress. Furthermore, cultures within this category favor strategic plans and have clear institutional objectives. A culture such as this could assist the $\mathrm{CDO}$ in their efforts. The third culture is the developmental culture, which strives for an environment that encourages the "potential for cognitive, affective, and behavioral maturation among all students, faculty, administrators, and staff' (Bergquist, 1992, p. xiv). It takes into consideration the "needs of the institution" (p. 74) as it addresses needs for change. The fourth culture Bergquist's (1992) identified is the culture of advocacy. This culture seeks an environment that is based upon fair and transparent polices as it relates to the distribution of resources. 
A further example of cultural typologies in higher education is a framework provided by Robert Birnbaum. Birnbaum's (1988) cultural models include the collegial, bureaucratic, political, and anarchical cultures. He argues that it is possible for all four models to operate within a university at the same time, which can benefit CDOs who use a multiple lens approach to understanding organizational culture. In comparison to Bergquist's (1992) typology, Birnbaum's (1988) models go deeper into the unique features of higher education and place greater emphasis on the relationship of the university to the external environment. In Birnbaum's (1988) typology, the collegial culture values shared governance and power among faculty and selected stakeholder representatives versus one single person or office. It is non-hierarchical and favors consensus decision-making. It also relies heavily on tradition and precedent. The bureaucratic culture follows formal rules and policies that are supposed to ensure consistency, fairness, and efficiency. Decision-makers in this culture seek to reduce uncertainty and ambiguity with clear guidelines for rules and polices. In the political culture, negotiating is front and center and those engaging in negotiating represent specific interests and coalitions. Those operating in the political culture rely on support from various groups in exchange for future support. The last culture represented in Birnbaum's (1988) model is the anarchical culture, which borrows from the work of Cohen and March's (1974) organizational ambiguity. The anarchical culture reflects the unusual organizational structure and decision-making process of universities. In the anarchical culture goals are vague, ambiguous, and often-in conflict with one another, thus creating an opportunity for individuals and groups to interpret their own meanings and derive at their own solutions. Decision-making is fluid and is the result of the system 
versus any outlined policy or procedure that would be present in the bureaucratic culture (Cohen \& March, 1974). This culture could be the most harmful to a change effort led by the $\mathrm{CDO}$ because there is no clear accountability, support, or vision from the institution.

Idiographic approach. In contrast to the nomothetic approach; William Tierney's work on organizational culture in higher education uses an idiographic approach. Idiographic approaches to culture focus on the cultural differences and uniqueness of each organization rather than using a set typology. As such, Tierney focuses on how a university's unique culture impacts many of its attitudes and behaviors for change (1988). Utilizing participant observation methods and in-depth personal interviews, Tierney developed a framework for assessing a college or university's culture. Tierney's work identified six elements that need to be analyzed in order to get at the organizational culture of a university. These include the environment, mission, socialization, information, strategy, and leadership of the institution. Specifically, how a university defines it's environment, the context and valued placed on the university mission, how members in the university are socialized into the organization, the control and value placed on information, the method in which decisions and strategy are derived and the expectation the university has of it's leaders. Tierney (1988) argues this methodological approach is better suited at getting at the underlying assumptions operating deep within the organization.

The key difference of Tierney's (1988) work and the works of Birnbaum (1988) and Bergquist (1992) is that a cultural analysis is derived from what is observed through in depth qualitative methods rather than imposed on from existing nomothetic typologies. The framework allows for the researcher to capture the distinctive aspects of the 
university that is being studied, yet could be used as the basis of comparison with other institutions. Another difference is that Bergquist's models were hypothesized but never empirically tested. Birnbaum's models; however, were developed after extensive research on the cybernetics of academic organization and leadership (1988).

Scholars examining organizational change in higher education have used both approaches to assess the influence and significance of culture in organizational change. In a 2002 study, Adriana Kezar and Peter Eckel examined how culture impacted the institutional transformation process. Kezar and Eckel (2002) asked the question "is culture the modifying element of change or the subject of the modification" (p. 438). To answer this question, Tierney and Bergquist's approaches were used side by side to understand the culture in six different institutions undergoing a change effort. Their results found that change strategies seemed to be most successful if they could be understood within their cultural context and did not violate cultural norms. Institutions that violated cultural norms were less successful. They also argue that more empirical studies on the impact of organizational culture on change efforts in higher education need to be conducted.

In summary, the culture of an organization is linked to the change effort (Kezar \& Eckel 2002; Kotter, 1996). How a CDO makes sense of the organization can be approached in one of two ways. They can use existing cultural typologies, in a nomothetic approach or they can conduct their own analysis of the institutional culture using an idiographic approach. Kezar and Eckel's (2002) research suggests using both approaches in a multiple lens assessment. This could be helpful to CDOs attempting to lead transformational change in the organization. 


\section{Models for Diversity}

As described in the previous sections, CDOs are expected to lead a diversity agenda that is transformative. A diversity agenda attempts to integrate diversity into the structure, culture and fabric of the institution so that it becomes institutionalized as a permanent feature of the university (Curry, 1992; Davis, 2002, Kezar, 2007). However, diversity can conjure different meanings to different audiences, which impacts how the $\mathrm{CDO}$ and the campus at large approaches transforming the institution into a diversity leading enterprise. Diversity models, such as the ones that will be discussed in this section, can help a CDO and the institution orient their diversity agenda to the institution's understanding of diversity. To help understand the complexity of diversity in higher education, Damon A. Williams and Charmaine Clowney (2007) identified three models commonly used in higher education. They are the Affirmative Action and Equity Model, the Multicultural and Inclusion Model, and the Diversity, Learning, and Research Model. In these three models, "diversity is driven by social imperatives, educational imperatives, and organizational performance" (Williams, 2013, p. 203). This section of the literature will further elucidate on these three models and examine the research that supports them. In addition, this section of the review explores another model, the Inclusive Excellence Model, which incorporates various elements of the Williams and Clowney models and provides clear steps for CDOs and other institutional leaders to follow in their change process. In this study, all three CDOs used various aspects of these models to inform their work at their respective institutions.

\section{Affirmative Action and Equity Model}


The first model Williams and Clowney (2007) theorize is the Affirmative Action and Equity Model. According to the researchers, the model was "developed to eliminate overt barriers of exclusion to higher education and increase the numbers of minorities, women, and other protected groups enrolled in and working on college and university campuses" (William \& Clowney, 2007, p. 4). This model emerged in the 1950s after legal rulings such as Brown v. the Board of Education, 1954 and subsequent Civil Rights Act legislation in the 1960s establishing protected classes, such as women and racial minorities were passed. The basis for the model and the organizational structures that formed as a result were to fulfill legal obligations, such as compliance and to address issues of social justice that the external environment demanded (Williams \& Clowney, 2007). As Williams and Clowney describe it, the model was "meant to spur change in demographic representation and eliminate overt discrimination" (2007, p. 4). Because of this historical context, the diversity rational for this model is rooted in social justice and its focus is to change the profile of the institution (Williams \& Clowney, 2007). Its efforts are directed at underrepresented groups of students, faculty, and staff and increasing the compositional diversity within these groups (Williams \& Clowney, 2007).

The most significant and visible aspect of the model is affirmative action policies related to undergraduate and graduate admission. These policies are regularly under public scrutiny and some would argue have limited how universities look and act on diversity strategies (Chang, 2002). Beginning with the Supreme Court case, Regents of the University of California v. Bakke, 1978, affirmative action policies have been debated, threatened, and challenged not only in the courts but also by voters (California, Michigan, Washington). In the Bakke case, a prospective White medical student sued the 
University of California for denying him admission into the University of California,

Davis School of Medicine. Bakke claimed that the university had violated his rights protected under the California Constitution, Title VI, and the Equal Protection Clause of the Fourteenth Amendment (Chang, 2002). What makes Bakke noteworthy is the opinion written by Justice Lewis Powell that defended the right of universities to consider race as a limited factor in building a student body. This often cited interpretation contended that universities have the right, protected under the First Amendment to make their own decision about the educational purpose of the institution, including the selection of its student body. Furthermore, Justice Powell argued that diversity is "essential to the quality of higher education" and universities using race-conscious admissions practices "when narrowly tailored, serve a compelling state interest" (Regents, U.S. 312; 98 S. Ct., 2760). Narrowly tailored in this sense, "means that race is used no more than is necessary to achieve diversity and that it is only one of many factors being used" (Gurin, et al., 2002, p. 331).

However, the argument of diversity serving a "compelling interest" and the meaning of "narrowly tailored" has been debated since 1978 and as recently as Fisher $v$. University of Texas, 2013 and the U.S. Supreme Court ruling upholding Michigan voters right to ban race-conscious practices in public higher education institutions in 2014. Some scholars argue that too much emphasis on compositional diversity achieved via affirmative action is limiting. Mitchell Chang (2002) emphasizes that in light of legal actions and challenges, it is critical for researchers and practitioners to move away from arguments that solely look at admissions practices to make diverse and inclusive institutions. Chang (2002) argues that the diversity discourse needs to be much broader 
to look at the entire manner in which diversity impacts the educational environment. Jonathan Alger, associate counsel for the American Association of University Professors writing in 1997 agrees. He stated that criticisms on race-based affirmative action policies have partially succeeded because "universities have failed to establish the fundamental link between diversity and their educational missions" (1997, p. 21). Chang (2002) argues that rather than focusing on "preserving" an institutional practice, i.e. affirmative action, universities need to be more conscious of "transforming" colleges and universities toward diversity. He urges scholars to look beyond admissions practices as a strategy to create diversity. Rather, he suggests universities utilize student engagement theories to create diversity and equity-minded students. Chang advocates that scholars and practitioners be deliberate in designing diversity agendas that provide reinforcing experiences where students' learning and development grow. Most importantly, Chang contends that universities need to seek change at all levels (not just admissions practices and not just student composition) in order to transform the enterprise from traditional institutional practices and arrangements. He argues, "educational benefits associated with diversity emerge more often than not, out of institutional transformation and not out of preexisting ways of operating and behaving" (p. 132).

Debates surrounding affirmative action policies have forced educators to articulate clearly the educational purposes and proven benefits of diversity to dubious courts, voters, and select members of the academy (Gurin, et al., 2002). As such, there is empirical evidence demonstrating that racially diverse higher education environments are associated with positive intellectual and social outcomes (Astin, 1993; Chang, 1999; Gurin 1999; Gurin, et al., 2002; Smith, 1997). Patricia Gurin's research posits that 
student involvement in activities related to diversity and interactions with diverse people are essential to the developmental growth of young adults (Gurin, 1999). In expert testimony given to the U.S. Supreme Court, Gurin stated "higher education will be especially influential when its social milieu is different from the home and community background, and when it is diverse enough and complex enough to encourage intellectual experimentation and recognition of varied future possibilities" (p. 37). Similarly, the research conducted by Gurin et al. (2002) found that "a more diverse university environment stimulates a more active engagement in the learning process and results in the development of less automatic and more complex thinking about issues and causality, as well as in the greater learning that comes from this engagement" (p. 43). In addition, scholars such as Jeff Milem and Sylvia Hurtado posit that diversity helps the educational enterprise (Milem, 2003) by producing college graduates who are better prepared for participation in a democratic society (Hurtado, 1998, 1999). Milem claims diversity benefits the individual, institution, and society at large (2003). Using national longitudinal student data, Milem found that students who interacted with racially and ethnically diverse people and participated in classes and coursework that focused on diverse perspectives, were more likely to report increased levels of racial and cultural awareness, greater commitment to supporting racial understandings, and liberal attitudes to racial diversity and aspects of social justice (2003).

The Affirmative Action and Equity Model has worthy components to bring about institutional change but its scope and focus on underrepresented minorities, particularly students can be limiting if it is not broadened and linked to other aspects of the university. Additionally, this model relies heavily on student compositional diversity to 
create conditions for richer student learning outcomes. However, compositional diversity alone cannot transform a university (Chang, 2002). Finally, public universities that are barred by state law from using race, ethnicity, or gender as a factor in admission decisions are at a significant disadvantage if they were to use the Affirmative Action and Equity Model because they might not have the full capacity to recruit historically underrepresented students or provide student services to groups from specific racial or ethnic groups.

\section{Multicultural and Inclusion Model}

The second model identified by Williams and Clowney (2007), Multicultural and Inclusion Model offers a more modern definition of diversity and incorporates inclusion. As a result, this model may be more helpful to CDOs leading a transformational change process because it is more comprehensive and considerate of other forms of diversity aside from race and gender. The Multicultural and Inclusion Model stems from the political and cultural transformations of the 1960s and 1970s and expands concepts of diversity beyond racial and ethnic lines (Williams \& Clowney, 2007). The model is described as being:

motivated by a commitment to capitalize on the richness of different cultures to help the members of those cultures thrive within the context of a broad institutional environment that may in an active or passive way, resist their presence on campus (p. 6).

As such, the model often aligns services, programs, and offices that are directed at underrepresented or historically excluded groups. The Multicultural and Inclusion Model makes an important distinction between "diverse groups" and groups that are federally protected, which is the focus of the Affirmative Action and Equity Model (Williams \& Clowney, 2007). Services and offices for "diverse groups" can include support for 
students, staff, and faculty who identify as LGBTQ, undocumented, international, differently abled, first-generation, and members of religious minorities to name a few. The diversity agenda in the Multicultural and Inclusion Model strays from concepts framing diversity as a moral imperative needed to correct racial injustices and inequities. Instead it focuses on accepting cultural differences in a manner that establishes mutual understanding (Williams, 2013). Inclusion becomes the focus of wellcoordinated diversity-related efforts (Smith, 1997). Student Affairs departments on university campuses traditionally address these points in their work with undergraduate students (Harper \& Antonio, 2008) and strive for an inclusive campus climate. Research examining the experiences of diverse students in predominately White institutions supports the inclusion element of the model. For example, theories on the social capital of minority students (Yosso, 2006), the need for cultural validation in the classroom (Rendón, 1994), and environments that foster a sense of belonging (Hurtado \& Carter, 1997) advocate that institutions build a diversity agenda that is inclusive to the complex and multifaceted identities students, particular minority or marginalized students, poses.

Williams (2013) cites that cultural centers, minority affairs offices, ethnic-specific student organizations, and resources for religious minorities, such as prayer rooms for Muslim students, and other "safe spaces" are outcomes of the Multicultural and Inclusion Model. He also argues that some within higher education consider the model and practice of building separate offices and centers, as the "balkanization" or "ghettoizing" of diversity whereby programs, offices, and services are subdivided into silos (Williams, 2013). In the most extreme sense, efforts to be inclusive of minority groups in predominately White campuses can appear to critics to be creating more segregated 
campuses with for example, Mexican-American students clinging to Raza resource centers or services (Williams, 2013). Indeed, research has shown that despite cultural centers and other resources directed at multicultural and minority students, there still could be an overall pervasiveness of Whiteness in space, curricula, and activities (Hurtado \& Harper, 2007). This also speaks to the significance of campus climate, which is an important aspect to be mindful of if the model is to be successful.

\section{Learning, Diversity, and Research Model}

The last model that Williams and Clowney (2007) introduce is the Learning, Diversity, and Research Model, which offers the most contemporary definition for diversity in higher education and links diversity to the academic enterprise. In Williams and Clowney's original work from 2007, the model is referred to as, "Learning and Diversity." However, Williams (2013) he renamed the model as "Learning, Diversity, and Research" to create stronger ties to the academic mission of higher education. As the name suggests, the Learning, Diversity, and Research Model is focused on integrating diversity into the curriculum and promoting research on diversity issues (Williams 2013; Williams \& Clowney, 2007). The researchers place this model's origins in the late 1990s, which "explains at long last the educational and social benefits of a diverse student body as well as the scholarship opportunities for advancing research around issues of diversity, equity, and inclusion" (2013, p. 148). Indeed, much of the research described in the Affirmative Action and Equity Model to defend practices of using race or ethnicity as a factor in admissions selections emerged during this period. This research demonstrated the benefit of diversity for all students, not just minority students, but White students as well (Astin, 1993; Chang, 1999; Gurin 1999; Gurin et al., 2002; 
Smith, 1997). As Williams describes it, empirical studies grounded in cognitive and social psychology supported the "compelling interest" argument by Justice Powell (2013). In this context, Williams and Clowney attribute the significant driver for the model to be dynamics taking place during the era of the University of Michigan Supreme Court decisions (Gratz v. Bollinger, 2003 and Grutter v Bollinger, 2003), which coincided with shifting demographics, globalization, and the need for a diverse and culturally competent workforce (Williams 2013; Williams \& Clowney, 2007).

Williams (2013) posits that the Learning, Diversity, and Research Model moves beyond discussions of access and equity for historically underrepresented groups found in the Affirmative Action and Equity Model and parts of the Multicultural and Inclusion Model. Rather, this model takes a broader approach to strategically align diversity into the mission of the university (Williams, 2013). As such, key indicators of the model are intentional links to the academic programs of the institution, which he claims "moves the diversity debate from the margins of the institution into the center in terms of academic teaching and learning" (p. 150). Furthermore, he argues with this model universities are better aligned to carry out the Association of American Colleges and Universities' "essential learning outcomes," which include integrative learning, inquiry learning, global learning, and civic learning (2013). Thus the rationale for this particular model is focused on the educational value of diversity, whereas the Multicultural Model and Affirmative Action and Equity Models are rooted in values that are oriented towards social justice (Williams, 2013; Williams \& Clowney, 2007). Because the motivation is diverse learning environments and engagement, the model tends to be even more inclusive than the Multicultural and Inclusion Model. Diversity in this model can be 
defined as student experiences and backgrounds that include international students, transfer students, student veterans, former foster youth, and student parents. One way to operationalize this type of learning is through, general education diversity requirements, intergroup relations sections, living learning communities, and civic engagement or service learning opportunities (Williams, 2013).

As in the other three models, Williams and Clowney highlight limitations and critiques. Williams (2013) points out that some critics find fault with the model because they claim it waters down important conversations about structural racism and existing inequities in higher education, particularly among African American, Chicanos and Latinos, and Native Americans students. Furthermore, he adds that some claim that while working in this model institutional leaders can choose not to address these more difficult challenges and instead elect to work on issues that are less politicized, such as student veterans (Williams, 2013). Sylvia Hurtado addressed this very point in a presidential address to members of the Association for the Study of Higher Education in 2007. She suggests that research supporting the educational benefit of diversity is not to take away or ignore existing problems of inequality, but rather is for,

the production of citizens for a multicultural society that can result in leadership with greater social awareness and the complex thinking skills to alleviate social problems related to the complexities of inequality. The end goal is the improvement of education for students from different racial, economic, and religious communities who must work together to achieve a vision of the pluralistic democracy we aspire to become (2007, p. 193).

This example is illustrative of Williams and Clowney's point, that universities working on their diversity agendas will often have overlapping objectives that reflect different aspects of the three organizing models (2007). Indeed this tends to be the case in the three case sites of this study. Williams (2013) affirms that working in the Learning, 
Diversity, and Research Model does not preclude institutions from making serious institutional commitments to address issues of access or equity but rather opens the conversation for broader input (2013).

\section{The Inclusive Excellence Model}

Williams and Clowney's three models of diversity are helpful to CDOs and other campus leaders who are considering how their campuses have historically addressed and approached diversity. They are also helpful because they can help the CDO identify a new diversity model to base their transformational change process on. However, the Inclusive Excellence (IE) Model is yet another option for universities to consider. The IE Model helps institutions transform their campus into more diverse, inclusive, and equitable environments and provides a more comprehensive approach with clearer steps for implementation and accountability (Williams, Berger, and McClendon, 2005). Williams, Berger, and McClendon define the IE Model as a model where "diversity is no longer envisioned as a collection of static pieces - a programmatic element here, a compositional goal for the student body there" (p. 3). Rather they posit that within the IE Model,

diversity is a key component of a comprehensive strategy for achieving institutional excellence - which includes, but is not limited to, the academic excellence of all students in attendance and concerted efforts to educate all students to succeed in a diverse society and equip them with sophisticated intercultural skills (p. 3).

Furthermore they argue that "diversity, as a component of academic excellence is essential to ensure higher education's continuing relevance in the twenty-first century" (p. 3). 
The IE Model involves an "integrated framework" that is considerate of environmental factors such as, shifting demographics, political and legal dynamics, societal inequities, and workforce needs (Williams, Berger \& McClendon, 2005). It also reflects on the challenges of expanding access and maintaining quality in higher education. Williams, Berger, and McClendon suggest doing this by breaking down the key elements of organizational culture, which they argue are rooted in the mission, vision, values, traditions, and norms of the institution. They argue campuses need to reconcile unsupported notions that put inclusion and excellence at odds. The reliance on a "dominant industrial model of organizational values" that considers excellence to be exclusive "limits both the expansion of student educational opportunities and the transformation of educational environments" (p. 9).

IE accountability. A key feature of the IE Model is accountability. As such, Williams, Berger and McClendon (2005) devised an IE Scorecard to account for performance and to communicate progress to the wider campus community. Borrowing on the work of Estela Bensimon's (2004) data-driven, information-tracking Diversity Scorecard, the IE Scorecard "allows campuses to pinpoint where they are doing well and where they need to improve" (p. 19). It is a mechanism to drive and measure the organizational diversity change process. In the IE Scorecard, success is determined by examining the baseline, target, and equity goal. Williams, Berger, and McClendon break this down as follows; "the baseline involves information on the institution before the [diversity] intervention strategies are launched, the target involves what the institution is trying to achieve, and equity represents the ratio of the baseline to the target" (p. 23). 
This mechanism for measurement is applied to four goal areas; (1) access and equity; (2) campus climate; (3) diversity in the formal and informal curriculum, and (4) learning and development (2005). Williams, Berger, and McClendon believe that,

more than any other area, the access and equity indicator 'make sense' to campus leaders because it is concrete and quantitative. The remaining three areas are often more qualitative in nature and therefore more difficult to capture and assess. Assessment of all four areas, however, is necessary to form a more complete picture of an institution's current level of progress toward making excellence inclusive (p. 23).

With the outlined goal areas and methodology for tracking progress delineated, the IE Model can be most easily adopted into a template for a campus diversity agenda. The researchers acknowledge that strategy is at the heart of the IE Scorecard.

Capitalizing on levers for change, they argue that a number of factors must be present for the IE Model to transform a campus. These include strong leadership and accountability mechanisms; vision and buy-in from multiple levels within the organization, capacity building for long-term strategies; and the appropriate financial, technical, human, and symbolic resources available to drive the process (Williams, Berger, \& McClendon, 2205, p. 26-28).

\section{Additional Diversity Models}

The IE Model definitely includes aspects of William and Clowney's diversity models, given Williams involvement in developing both. In addition all four models borrow from existing diversity models that explore further ways to institutionalize diversity. It is important to reference these models before concluding this literature review because they demonstrate how scholars have contextualized the importance of diversity and inclusion for institutional transformation. One such model is Daryl Smith's 
framework for diversity (1997). After reviewing the extant research, Smith devised a framework of four distinct but interrelated dimensions to help educators define and account for their diversity efforts (2009). These four dimensions are access and success; campus climate and intergroup relations; education and scholarship; and institutional viability and vitality. This model was utilized in the five-year Campus Diversity Initiative study funded by the James Irvine Foundation between 2000 and 2005, which Smith was a lead researcher. The study was designed to help campuses build capacity to evaluate their diversity strategies. Findings resulted in a monograph for higher education leaders to use in developing a diversity agenda for institutional change (ClaytonPedersen, Parker, Smith, Moreno, Teraguchi, 2007). One key recommendation from the study was to appoint a CDO to lead the campus' diversity efforts.

Hurtado, Milem, Clayton-Pederson, and Allen (1999) devised a framework for assessing campus climate, which is also a key element in institutionalizing diversity and inclusion. They argue a campus' racial climate needs to be understood from various student perspectives before diverse learning environments can be enacted. Their model consists of four elements that make-up the institutional context. They are the historical legacy of inclusion and exclusion on the campus; the structural diversity (compositional diversity); the psychological climate, which relates to the feelings and emotions of the institution's inhabitants; and the behavioral dimension, which includes the interactions and practices of the institution (1999). Additionally, they have determined that a campus' institutional context is influenced by the external factors of government and policy and socio-historical contexts (1999). They argue that improving a campus' climate may involve "fundamental institutional changes" that results in a "shift in 
thinking about how diversity is central to the institution's overall priorities for teaching and learning (p. v). Milem, Chang and Antonio who added a fifth dimension revised the model in 2005 . The new dimension they added, referred to the organizational and structural condition of the campus, which contains the campus' current policies. These policies may either support or hinder a campus' diversity efforts and subsequent diversity agenda.

Throughout this study, the CDOs and institutional leaders interviewed referred to various aspects of Smith's diversity model, the Hurtado et.al, campus climate model, and the IE Model. Elements of Williams and Clowney's three models also appeared in this study, most notable the Learning, Diversity, and Research Model and the Multicultural and Inclusion Model. References to these models will be discussed more thoroughly in Chapters 4 and 5.

\section{Summary}

In closing, the literature presented in this review is helpful in understanding the role CDOs play in transformational change efforts supportive of diversity and inclusion. However, as evidenced by this review, the current literature offers few examples of empirical studies that pertain to the relationship of CDOs and transformational change efforts. Rather the two topics are studied in separate contexts, i.e. literature focusing on CDOs or literature pertaining to organizational change in higher education. Furthermore, how CDOs lead a transformational change effort also pertains to how the campuses define diversity and the models they use to lead their organizations. Aside from those mentioned in this literature review, few scholars have linked organizational change and diversity to institutions that have successfully navigated these change processes with a 
CDO. This study attempts to contribute new data on how institutions with a CDO lead a transformational change effort supportive of diversity and inclusion. 


\section{CHAPTER THREE}

\section{METHODOLOGY}

\section{Methodological Overview}

The purpose of this study was to examine the role CDOs play in institutionalizing diversity and inclusion efforts that lead to transformative change. A further purpose is to understand how the three campuses in the study have institutionalized diversity campuswide so that diversity is a sustained institutional effort with identifiable and measurable outcomes. The role of CDOs and the institutionalization of diversity and inclusion in higher education are important aspects to study in tandem because they shed light on the actual versus perceived operationalization of diversity efforts in higher education. In this study, institutionalization is defined as "establishing a standard practice or custom in a human system" (Curry, 1992; Kramer, 2000) and is considered the final phase of a change process toward a particular goal (Curry, 1992). In this study the goal is diversity and inclusion and the change effort is lead by the CDO. The research design and methodology used in this study was purposefully developed to answer the following three research questions:

$\mathrm{RQ \# 1}$. What role do CDOs play in institutionalizing diversity and inclusion in US universities?

RQ\#2. How do three exemplar universities know they have institutionalized diversity and inclusion? What are their outcome measures and who is involved in these efforts? 
$R Q \# 3$. What key strategies contribute to successful institutional efforts to sustain diversity and inclusion at three exemplar universities? What is the role of the CDO in these efforts?

This chapter describes how this study was conducted through detailed accounts of the research design, methodology, data collection, and data analysis. The chapter concludes with a pertinent discussion on the role of the researcher, the trustworthiness of the data, and the delimitations and limitations of the study.

\section{Research Design}

The research design for this study was broken down into two linking phases consisting of a questionnaire and a multiple case study analysis. The questionnaire served as the launch pad of the study and was instrumental in the identification of the case study sites eventually researched. Phase two, the examination of three case study sites, yielded the major findings of this study and is subsequently the area where the most time, attention, and analysis was deployed. Figure 1 illustrates the design of the research study. A detailed breakdown of each phase is described in the sections that follow. This includes the identification of participants, a description of the data collection processes and finally, the methodology used to analyze the data. 


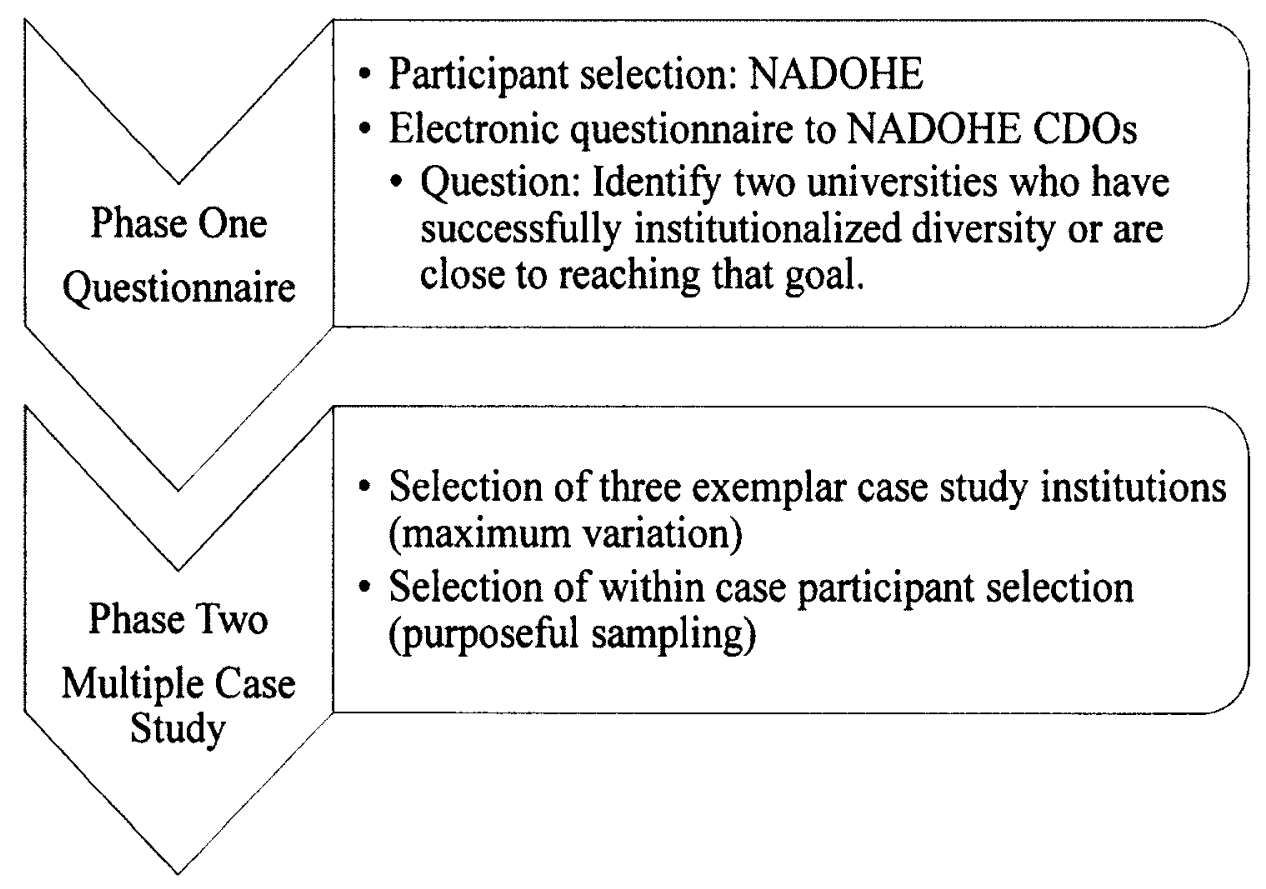

Figure 1: Diagram of Research Design.

\section{Phase One: Participant Selection}

As stated, the questionnaire served as the launch pad for the study. An organization that lent itself extremely well to the topic of study was identified. This was the National Association of CDOs in Higher Education (NADOHE). NADOHE is an organization that consists mostly of CDOs and other higher education professionals engaged in diversity work at various types of colleges and universities throughout the country. To support the validity of this study, it was important to have current CDOs assist in the identification of exemplar institutions to be studied. As a researcher, who is not a CDO, it was important to involve professional individuals engaged in diversity work at the highest institutional levels that are informed of the outcomes of institutional transformation toward diversity.

NADOHE was chosen for this study because of its focus "to lead higher education toward inclusive excellence through institutional transformation" and the 
concentration of CDOs as its members (NADOHE Mission Statement, 2014). The organization emerged from the American Council of Education's (ACE) Center for Advancement of Racial and Ethnic Equity. It became a professional organization of diversity officers at the ACE national conference in 2006. It describes its' purpose as serving "as the preeminent voice for diversity officers in higher education by supporting [its members] collective $\square$ efforts to lead [members'] institutions toward the attainment of the following goals:

- Produce and disseminate empirical evidence through research to inform diversity initiatives

- Identify and circulate exemplary practices

- Provide professional development for current and aspiring diversity officers

- Inform and influence national and local policies

- Create and foster networking opportunities (NADOHE Mission Statement, 2014)

At the time of this study, NADOHE consisted of 129 individual members and 171 institutional members (Appendix A). Individual members join the organization separate from affiliated institutions, i.e., graduate students or higher education professionals and researchers. Individual members may also be those persons not affiliated with a university but desire to keep informed about diverse issues in higher education. An example of such a person may be an independent consultant or distributor with certain training products.

Since it's inception, the organization has steadily grown. Over the last two years membership has increased by $18 \%$ (NADOHE President's Message, 2014). It is an organization, intended for senior level diversity officers, which is generally defined as 
those individuals who report to the president or provost of an academic institution and have oversight for campus-wide diversity efforts (Williams, 2013). Despite its young age, NADOHE has quickly become a respected professional organization of scholars, policy makers, and practitioners in the field of higher education. It produces the Journal of Diversity in Higher Education, which is a quarterly publication jointly produced by NADOHE and the American Psychological Association. Its editors include twenty-seven leading scholars in higher education policy, theory, and research. The journal "publishes empirical research, literature reviews, and evaluations of promising practices and policies that support efforts to transform institutions, inspire colleagues, engage campus, governmental, and private sector leaders, and articulate culturally competent outcomes" (Journal of Diversity in Higher Education, 2014). Additionally, NADOHE provides policy briefings, white papers, and webinars on special topics and current events impacting higher education related to diversity. It holds an annual national conference that has seen increased attendance since its first conference in 2008 (NADOHE History, 2014). NADOHE supported the querying of its members for purposes of this research study in the fall of 2013 .

\section{Phase One: Data Collection}

The questionnaire was administered using software from Campus Labs. Campus Labs is a secure and trusted assessment company in the higher education profession that allows users to create electronic surveys and questionnaires. Data collected from the questionnaire was only accessible to the researcher. Data submitted by the NADOHE participants was collected confidentially without any personal identifying information. With NADOHE's permission, the questionnaire was sent electronically two times via 
email from the researcher's university email account on September 24 and on October 21, 2013. Both times the questionnaire was sent, it was delivered to two separate NADOHE managed listservs. The first listserv contained the emails of all current NADOHE members who had an email address on file with the organization. At the time the questionnaire was administered, this included 144 email addresses. The second NADOHE listserv contained the emails of members who self-identified as CDOs and opted-in to this special audience listserv. At the time the questionnaire was administered, the CDO listserv contained 55 email addresses.

Questionnaire design. To facilitate the creation of a list of exemplar institutions from which to study, a fifteen-question electronic questionnaire was designed to be completed by current members of NADOHE with a valid email address on file (Appendix B). The questionnaire served two complimenting purposes. The first purpose was to gather data on what the CDO position entails in higher education in general and the second, to form a list of exemplar institutions that could then be used to select the case study sites. The first question of the questionnaire was designed so that only CDOs would complete the survey and participate in the study. The definition of a CDO used in the questionnaire was guided by the current literature that defines a CDO as someone who has a seat at the president and/or provost's cabinet, directs campus-wide diversity efforts, has some level of authority and responsibility for holding departments and units accountable for diversity efforts, and is generally seen as the "face of diversity" at the institution's highest level (Clayton-Pedersen, et al., 2007; Williams \& Clowney, 2007; Williams, 2013). Individuals who received the survey that were not CDOs, were thanked 
for their willingness to participate but were kindly directed not to continue with the survey.

After the first question self-identified CDOs were directed to answer several multiple-choice questions to give context and background about the variation and complexity of their role and home institutions. Participants were asked to describe the student population size, the degree granting type of institution and whether or not the university or college was public or private. Another set of questions asked respondents to describe their position. The purpose of these questions was to get additional context for the variety and similarities within the $\mathrm{CDO}$ position. Questions included the number of units reporting to the $\mathrm{CDO}$, their operating budget, title and other appointments they hold in addition to $\mathrm{CDO}$.

The last set of questions aided in the identification of the case study sites. The CDOs were asked to identify two institutions of higher education they felt had been successful at institutionalizing diversity or are close to reaching that goal. Respondents could list their own institution. Respondents were then asked to select three outcomes from a list of factors higher education scholars (Hurtado, et al., 1998, 1999; Milem, Chang, \& Antonio, 2005; Clayton-Pedersen, et al., 2007; Smith, 1997, 2009; Williams, Berger, McClendon, 2005) have identified as either positive outcomes or critical elements necessary to achieve welcoming and diverse institutions. This list included the following options:

- Compositional diversity among the students body (undergraduate and graduate)

- Compositional diversity among faculty 
- Compositional diversity among staff

- Diverse curricula (majors, minors, GE requirements)

- Equitable retention and graduation rates for underrepresented minority students

- Institutional policies that promote diversity

- Institutional mission statement that delineates the type of diversity the campus is striving for and includes language that also addresses inclusion and equity

- A president that champions diversity publicly

- A CDO position

- A campus-wide strategic plan for diversity

- Support and buy-in from the local community on diversity initiatives

Respondents were also given the opportunity to list a different outcome measure or factor not included in this list.

Questionnaire analysis. Thirty-nine respondents completed the questionnaire, of which 33 identified themselves as CDOs. Given that the NADOHE CDO listserv contained 55 emails at the time the questionnaire was sent, this was considered a substantial enough response rate to utilize for this study. The data collected from these respondents were analyzed and assessed using basic descriptive statistics. An account of the questionnaire's major findings can be found in Appendix C. Analysis used to determine the case study sites and within case participants are outlined in the next section, phase two.

Phase Two: Participant Selection 
A case study is defined as a single unit or a " bounded system" (Merriam, 1998). In qualitative research, multiple case studies yield greater opportunity to strengthen the "external validity" of findings from a single case study to multiple units, thus bolstering opportunities for some generalizability (Yin, 205). The rational for three case studies was determined to produce enough opportunity to examine potential commonalities and differences among the unique experiences evidenced at each institution. Similarly, three case studies allowed for contrast against institution type (e.g. enrollment size, religiously affiliated, private or public) and geographic location. While this study contains a small sample of three cases, Patton (2002) reminds us that although one cannot generalize definitively from a small sample or a few cases, "one can learn from them - and learn a great deal, often opening up new territory for further research" (p. 46). In addition, three case studies were determined to be a sufficient amount to engage in "specificity of focus" (Merriam, 1998), which in this study means focusing on how the case study sites institutionalized diversity. Three cases was also a manageable amount to balance against real-world constraints such as time limitations and lack of financial resources.

Case study sites. Based upon the responses collected from the questionnaire, a list of universities was developed to use in the selection of the three case study sites for this study. The three higher education institutions selected for the case studies were determined using maximized variation sampling. Patton (2002) argues that even in smaller sample size qualitative studies, findings from "a small sample of great diversity" results in "important shared patterns that cut across cases and derive their significance from having emerged out of heterogeneity (p. 172). Thus, this methodology of sampling was applied to the list of responses gathered from the CDOs who identified two colleges 
or universities that had been successful at institutionalizing diversity or were close to achieving that goal. This question generated a list of 31 distinct institutions. All were four-year universities within the continental United States. From this list, institutions identified more than once became my focus for selecting the case study institutions.

The shorter list of institutions identified more than once contained six universities with variation in geographic location, total student enrollment size ${ }^{3}$, and type, i.e., public, private, or religiously affiliated. The smallest institution, a private university had a total student enrollment of 2,362 , while the largest university, a public research university had a total student enrollment of 50,627. Two of the universities were located in the Southeast, while the remaining institutions were evenly distributed in the South, Midwest, East Coast and West Coast. Of the six universities, three were private institutions and only one was religiously affiliated. The remaining four universities were all public institutions. Among the six universities, a single university was identified multiple times, more than any other university. This university, a large public research located in the Midwest immediately became of interest to include in the sample. To get maximum variation with the remaining two universities, one west coast institution religiously affiliated with a small student enrollment and one large public research university located in the South were selected. Next each institutions' website was investigated to look for traces of institutional efforts to lead campus-wide diversity efforts. Evidence of diversity programs and committees, a diversity plan, and a CDO were some of the aspects investigated to ensure there was enough material evidence present to advance the research questions. In conclusion, all three universities had robust

\footnotetext{
${ }^{3}$ Total enrollment includes undergraduate and graduate students.
} 
websites outlining their various efforts and organizational structure. Upon confirmation of this evidence, the three case study institutions were finalized. The following table lists the three case study universities by their pseudonyms and includes their geographic location, size, and some of the Carnegie Classifications ${ }^{4}$ to describe the institutional type. Table 1.

Breakdown of Case Study Universities

\begin{tabular}{|c|c|c|c|}
\hline$\frac{\text { Case Study }}{\underline{\text { Site }}}$ & $\frac{\text { Geographic }}{\text { Location }}$ & $\begin{array}{l}\text { Total Student } \\
\text { Enrollment Size }\end{array}$ & Institutional Type \\
\hline $\begin{array}{l}\text { Francis } \\
\text { University }\end{array}$ & West Coast & $\begin{array}{c}9,000+ \\
\text { Majority } \\
\text { Undergraduate }\end{array}$ & $\begin{array}{c}\text { Private Not-for-Profit } \\
\text { University, Religiously } \\
\text { Affiliated }\end{array}$ \\
\hline $\begin{array}{l}\text { Central } \\
\text { University }\end{array}$ & Midwest & $\begin{array}{c}40,000+ \\
\text { Majority } \\
\text { Undergraduate }\end{array}$ & $\begin{array}{c}\text { Public } \\
\text { Very High Research } \\
\text { University }\end{array}$ \\
\hline $\begin{array}{l}\text { Morrill } \\
\text { University }\end{array}$ & South & $\begin{array}{c}50,000+ \\
\text { High Undergraduate }\end{array}$ & $\begin{array}{l}\text { Public } \\
\text { Very High Research } \\
\text { University }\end{array}$ \\
\hline
\end{tabular}

Within case participants. The premise for case study research is to focus on a single entity as a unit of investigation (Merriam, 1998). For purposes of this study, the unit became a single institution of higher education. As discussed, three universities were selected, which resulted in a multiple case study designed to provide "compelling interpretation" of the data (Merriam, 1998). Once the site was determined, purposeful sampling was used to identify the participants within the case. In order to use purposeful sampling within case, a set of conditions were identified to determine participants fitting

\footnotetext{
${ }^{4}$ The Carnegie Classification is the leading framework for recognizing and describing institutional diversity in U.S. higher education.
} 
within the framework of the study (LeCompte \& Preissle, 1993; Merriam, 1998). The following case participants and the rationale for their selection is explained below:

- The CDO was selected because the purpose of the research study was to understand their role in the institutionalization of diversity;

- The provost of the university was selected because he or she has either oversight of the $\mathrm{CDO}$ and/or has a close working relationship with the CDO; additionally it was assumed that as the chief academic officer they could speak to the institutional mission of the university and where diversity lies within that construct;

- Any campus leader, staff person, or student engaged in a campus diversity related-effort was selected in order to learn from those working alongside the CDO

- Any beneficiary of a campus diversity related effort to understand if they were aware of the campuses diversity efforts or the CDO.

Utilizing these conditions, the case sites diversity websites were reviewed to identify ideal candidates for interviews. Several candidates were identified and formed the basis of a list of potential interviewees. Because case studies and multiple case studies attempt to understand the phenomena occurring within the unit or organization being analyzed (Merriam, 1998), individuals outside of the university where not interviewed for purposes of this study.

As the foci of the study, each CDO was contacted first by email to explain the purposes of the study. As trust and acceptance was established, a list of possible in-case interviewees was shared with the CDO. Suggestions for additional names were asked for 
at each set for snowball sampling. The CDOs at each of the campuses affirmed several of the names previously identified and suggested new names to consider for the study. Using the criteria for participant selection and the results from the snowball sampling a final list of participants within case was derived for each institution case cite. In total 16 people were interviewed for this research study. A detailed breakdown of within case participants by case study institution is included here.

Table 2.

Breakdown of Study Participants

\begin{tabular}{|c|c|c|}
\hline$\frac{\text { Case Study }}{\text { Institution }}$ & Participants & Total Participants \\
\hline Francis University & $\begin{array}{c}\text { CDO } \\
\text { Provost } \\
\text { Undergraduate Student } \\
\text { Undergraduate Student } \\
\text { Faculty Member } \\
\text { Student Affairs Administrator }\end{array}$ & 6 \\
\hline Central University & $\begin{array}{c}\text { CDO } \\
\text { Provost } \\
\text { Graduate Student } \\
\text { Diversity Administrator } \\
\text { Diversity Administrator } \\
\text { Diversity Administrator }\end{array}$ & 6 \\
\hline Morrill University & $\begin{array}{c}\text { CDO } \\
\text { Provost } \\
\text { Student Affairs Administrator } \\
\text { Academic Affairs } \\
\text { Administrator }\end{array}$ & 4 \\
\hline
\end{tabular}




\section{Phase Two: Data Collection}

Merriam notes that data collection in case study research generally involves strategies of interviewing, observing, and analyzing documents (1998). In this multiple case study, all three methods of data collection were used. In preparation for the case site visits, data and information contained on each of the CDO websites' was reviewed. As exemplar institutions, all three campuses had varying but robust websites that communicated to internal and external campus community members about the CDO's office and the institution's overarching diversity efforts. Each website contained documents such as the campus' diversity agenda and strategic plan, reports regarding diversity-related efforts, and other helpful information such as resources for students, staff, and faculty. To help with the sorting and understanding of the information contained on the websites, a table was created to track and differentiate between the different materials contained on the websites. This table can be found in Appendix D.

Based upon data collected from the website analysis and content derived from the literature, semi-structured interview guides were created for each of the within case participants (Appendix E). However, because qualitative research can be iterative and data analysis can occur simultaneously with data collection, modifications to the interview guides were made throughout the data collection process as concepts, hunches, and themes appeared (Charmaz, 2006; Glaser \& Strauss, 1967; Merriam, 1998). Interviews were conducted at all three of the case study sites in order to meet the participants in their campus environments and to observe the behaviors of the extended campus community. Interviews were conducted for no less than 60 minutes and were recorded using a digital recorder. Prior to the interviews, the participants received a 
confirmation email with a short summary of the research questions guiding the study. At the start of the interviews each participant received the participant consent form. After each interview was transcribed, participants were sent a copy of the transcript for purposes of member checking.

In addition to the within case participant interviews, data was collected through observations during the three day visits at each of the case study sites. A campus tour occurred at two of the case study sites. Inclement weather prohibited participation in a campus tour at the third institution; however, a stay at a hotel located within the student union of the campus and resulted in rich observation of the campus dynamics at the highly utilized facility. Observation of a committee meeting held by one of the case sites diversity agenda writing groups took place. Informal memos drafted during the observational data collection and after interviews, assisted with participant interviews that had not yet taken place and with the simultaneous analysis of the data collected thus far (Charmaz, 2006). This again highlights the iterative and refining nature of qualitative research (Charmaz, 2006; Merriam, 1998). Additionally where available, pamphlets, flyers, and newsletters were gathered and read to look for further evidence of the campus' diversity and inclusion efforts.

\section{Phase Two: Data Analysis}

As described in the data collection section, simultaneous data analysis began as data was being collected. A constant comparative analysis was used during the data collection process. A constant comparative approach involves taking a unit of data and establishing analytic distinctions that allows comparisons at each level of analytic work (Charmaz, 2006). As data is collected it is continuously compared to other units of data 
(Merriam, 1998). This process began immediately with the first major analysis of the case sites' diversity websites and resulted in the previously mentioned website table (Appendix D). At the physical case sites, this process continued as observations were made between interviews, during campus tours, and as materials such as pamphlets, flyers, and newsletters were collected from the case sites. Quick and on the spot analysis were done to inform the next opportunity for data collection. These analyses were mostly captured in field notes.

Analysis of the in person interviews and field notes began immediately after transcripts from the interviews were member-checked by each participant. Initial coding was used in the first round of coding. Initial coding allows for the data to be broken down into discrete parts to be examined and then compared for similarities and differences (Saldaña, 2009). In the margins of the interview transcripts and field notes, codes were assigned. A list of over 125 codes was created during this process.

From the initial list of codes, categories and subcategories were generated using focused coding. Focused coding searches for the most frequent and significant initial codes (Saldaña, 2009). The literature was used as a guide in labeling some of the categories; however, some terms used directly by the participants were retained. This approach blended concepts from the literature and from the participants into one master list to get more accurate categories. After this process was complete, the list of refined categorized codes was vetted with an external reviewer familiar with organizational change and diversity in higher education. As a result of this discussion, a few minor refinements were made to the list of codes, which was then reapplied to the data from the 
interviews and field notes in the second cycle of coding. A qualitative research software program aided this process.

In the second cycle of coding, the new code list was used within case and a cross cases. The CDO and Provost interviews were analyzed both ways. A cross case analysis was done for these two groups to identify reoccurring and divergent themes within the positions. This was important because according to the literature the $\mathrm{CDO}$ and chief academic officer are important players in institutionalizing diversity. It was also important to analyze these two positions within case to understand local phenomenon occurring within the case study sites, which could then compared to the remaining two institutions in the study.

In summary, data analysis of the in person interviews and field notes served as the primary evidence for the findings discussed in Chapter 4. Data analyzed from the materials gathered at the case study sites and the websites served as background information and helped to guide me to the appropriate participants and in being able to ask site-specific questions about the university's efforts to institutionalize diversity and inclusion. The interview and field notes were more heavily utilized and generated the findings.

\section{Delimitations and Limitations}

Delimitations within the study exist and are worthy of noting. To begin the first phase of the study was limited to members of NADOHE only. This organization was selected because of its concentration of CDOs, which is a significant element of the study. As a professional organization with reputable ties in the higher education community, it was decided that using this single resource was adequately sufficient given 
limitations of time and financial resources. Despite this, it is worth noting that a CDO who was not a member of NADOHE at the time this study was conducted, was not part of the sample that participated in the questionnaire to identify the case study sites. As a result, it is possible that the list of recommended institutions to examine could have been strengthened with more CDOs participating in the questionnaire. Second, as mentioned in the within case participant selection, this study did not involve interviews with members outside of the university community who may have provided insight into the perceptions of the campus' institutionalization efforts. This could have provided another layer of stakeholders from which to gather feedback and to strengthen the findings. However, due to limitations already mentioned in time and financial resources, this group could not be included, as the selection of such members would have added more pressure on existing time constraints.

Despite attempts to design a thorough and robust research study, limitations also exist within the study. First, limitations on time and financial resources resulted in a single visit to each campus for a short period of time (three days). To adjust for this constraint, a great amount of time researching each case site was spent prior to arrival in order to make the most out of the time available. Additionally, a significant portion of the case site visit was spent in observation of the campus community in action to get a feel for the behaviors, climate, and tone of the campuses. Second, also due to the limitations of time and financial resources, the case study sample size and within case study participant sample are small. The effect of these limitations likely resulted selection bias of within case participants, as a large sample with a range of perspectives could not be reached. As a result, the within case participants were actively involved in 
the diversity efforts and were supporters of the campuses' initiatives. Therefore, most of the comments gathered generally reflect a positive and supportive point of view. With more time and financial resources, a wider within case sample could have been approached to get more variety within the responses to include those who may be less satisfied than those who were interviewed.

Notwithstanding the limitations, it must be understood that the findings from this study are not intended to be generalizable to every institution with a CDO or institution currently working on institutionalizing diversity. Similarly, it can also be argued that each case is highly localized and unique to its environment. Nonetheless, a multiple-case study provides information-rich cases that can be quite useful in similar contexts (Glesne, 2006; Patton, 2002; Yin, 2005). It is this richness of information that makes qualitative studies valuable to scholars and practitioners over a randomized large sample. However despite this attribute of qualitative research's ability to reach great depths of detail, one can never fully understand the experience of another person or an organization (Patton, 2002).

\section{Role of the Researcher}

Research, (qualitative or quantitative) has some level of bias within it based upon the researcher's prioritization of certain thoughts and ideas that come from the data (Merriam, 1998; Patton 2002). Furthermore, because the researcher becomes the instrument for collecting data in the study, the role of the researcher is significant (Patton, 2002; Kvale \& Brinkman, 2009). To this point, I share that my own personal being bears weight on this study. I am a former first-generation college student, member of a historically underrepresented minority group in higher education, and am currently 
working in higher education in an administrative role in Student Affairs. As such, my experiences collecting data and interpreting the data undoubtedly influenced this study. To bracket my own personal biases, I wrote notes to myself about lingering questions, ideas, or feelings I had related to the study and my own personal experiences before, during, and after the data collection and analysis. Most of this was related to my own professional work experience and my current university environment, which is in the process of trying to articulate a way forward in institutionalizing diversity on the campus. While going through this process in my current work environment helped me understand the process of institutionalizing diversity, I did not want it to overly influence my work on this study. Ideas or concepts that I thought would be helpful to my current institution were simply jotted down as notes to myself and kept in a separate file. Furthermore, as a professional who works regularly with historically underrepresented students and on issues pertaining to their academic success, I could not help but to compare student experiences observed in the study with student experiences at my home institution, which varied considerably based on institution type, geographic region, and historical legacies about the campuses. Again, I captured these feelings and questions in notes to myself contained in a separate file. In addition to addressing personal biases, I consulted regularly with colleagues and committee members within the field of study and outside by testing concepts and ideas as they emerged.

Despite this it should be acknowledged that the role of the researcher in this study is to understand a movement within higher education that is attempting to explain and address the efforts of CDOs to strengthen institutional diversity and inclusion. My professional work and area of focus in graduate school aligns with this mission; therefore, 
I believe studying efforts to institutionalize diversity is a worthy area of research. Where possible and appropriate, I used these experiences to probe deeper into the study to yield richer data. Despite the factors outlined in this section, I believe there is enough significance from the study to contribute to both the fields of CDOs in higher education and the institutionalization of diversity in US colleges and universities. 


\section{CHAPTER FOUR}

\section{FINDINGS}

\section{Overview of the Findings}

This chapter describes findings that emerged from sixteen interviews conducted at the three case study institutions and the researcher's field notes from the case site visits and information gleaned from supplemental materials such as pamphlets, flyers, and content analyzed from the case sites diversity websites. Participants in this study included the $\mathrm{CDO}$ and the Provost from each of the three universities and ten other participants that included various campus administrators from the office of student affairs and diversity divisions, faculty, and students (both undergraduate and graduate).

As a result of this study, three major findings emerged. First, the data indicated that the role CDOs play in institutionalizing diversity and inclusion is to be leaders in the change efforts on campus. As evidenced in this study, their role is to bring the wider campus along in its efforts to build inclusive and diverse universities in a variety of intentional ways. Furthermore, to lead change the CDOs in the study carry the message of diversity and inclusion to all corners of the university so it can permeate the wider organization and result in changed attitudes and behaviors supportive of diversity. The CDOs in this study do this by operating with intentional strategies that require relationship building when working with campus partners. They also understand that the change process has a long trajectory if it is to be deep and pervasive in the wider organization.

Second, data from all three campuses in the study suggested that the institutionalization of diversity is evident when attitudes and behaviors surrounding 
diversity and inclusion shift from traditional notions of diversity to broader understandings of the complexity of diversity and its benefits to the university. As part of this broader understanding the universities in the study gave additional attention to campus climate because it relates to how welcoming the campus is to diverse people. Thus with their more complex understanding of diversity, the campuses recognized that diversity efforts had to address inclusion. In addition, outcome measures used by the campuses to determine their institutionalization efforts varied among the case study sites and were not uniform per campus. However, the CDOs in each case study site worked with the units within their institutions to come up with appropriate outcome measures based on institutional data the CDOs analyzed and assessments they conducted.

Third, data from the study indicated that although the case study sites utilize various strategies and tactics to support their campuses' diversity and inclusion efforts, use of five key strategies emerged from the data at all three sites. The CDO's role is evident and integral to all five strategies. A detailed discussion of each strategy is included in this chapter; however, for purposes of introduction, the five strategies are noted here. First directed by the CDO, each campus used a campus-wide diversity plan to guide institutional efforts and to address accountability. Second, through the leadership of the CDO each campus analyzed the data carefully and took deliberate steps to understand what the data was really revealing about populations within the campus community. Third, each campus in conjunction with the $\mathrm{CDO}$ took deliberate steps to educate the campus using the data. They did this by sharing the data widely, which resulted in professional development trainings to discuss the findings and to identify related solutions. Fourth, the CDOs and the Provosts used incentives to reward and 
sustain knowledge, competencies, and efforts developed throughout the course of the institutionalization. Fifth, each of the campuses assessed the external environment to understand how external forces could assist or might subvert ongoing diversity and inclusion efforts led by the CDO. These findings and related strategies are important because each one and the evidence supporting them provides guidance and context for campuses to consider as they engage in attempts to institutionalize diversity and inclusion at their institutions. These findings also go deeper than the existing literature on CDOs and provide specific examples of how the CDOs and their respective campuses in the study are attempting to transform their institutions to be more diverse, welcoming, and inclusive of multiple people and identities.

This chapter begins with a brief review of the study's purpose and research questions, followed by a discussion of the CDO participants in the study. Findings from the study are presented in relation to the research questions. This format was chosen to provide organizational context and because the research questions build upon each other and illustrate the involvement of the $\mathrm{CDO}$ throughout.

\section{Review of Purpose of the Study and Research Questions}

The purpose of this study was to examine the role of CDOs in the institutionalization of diversity and inclusion at their respective institutions. The role of CDOs and the institutionalization of diversity and inclusion in higher education are important aspects to study in tandem because they shed light on the actual versus perceived operationalization of diversity efforts in higher education. In this study, institutionalization is defined as "establishing a standard practice or custom in a human system" (Curry, 1992; Kramer, 2000) and is considered the final phase of a change 
process toward a particular goal (Curry, 1992), which in this study is openness to diversity and inclusion. To institutionalize diversity and inclusion means "leaders are engaging in the process of moving a diversity agenda forward to institutionalize a new way of doing work" (Kezar, 2007, p. 415). Characteristics typically associated with an institutionalized practice are often defined as routine, widespread, legitimized, expected, supported, permanent, and resilient (Kramer, 2000). During the institutionalization process, capacity is built, support is cultivated, and system integration is facilitated (Kezar, 2007). To this end, the study was designed to conduct research at three exemplar universities, where there were CDOs who have had success at institutionalizing diversity and inclusion on their respective campuses. In phase one of this research, feedback from current CDOs in the field of higher education provided the sample from which to identify the three case study sites. In phase two, based on intentional sampling, within case participants were identified at each of the universities for qualitative interviews.

Once decisions were made about campus selections, data collection commenced at all three campuses that had institutionalized diversity and inclusion strategies or have had some success in leading campus-wide diversity and inclusion efforts that had permeated through the wider institution. After data collection was complete and a thorough and careful analysis of the data was conducted, themes emerged from the data in relation to the three research questions:

RQ\#1. What role do CDOs play in institutionalizing diversity and inclusion in US universities? 
RQ\#2. How do three exemplar universities know they have institutionalized diversity and inclusion? What are their outcome measures and who is involved in these efforts?

RQ\#3. What key strategies contribute to successful institutional efforts to sustain diversity and inclusion at three exemplar universities? What is the role of the CDO in these efforts?

As a result of the purpose and design of the study, data and subsequent themes displayed interconnected qualities, mainly due to the fact that the CDO's role and influence was prevalent throughout the data. For this reason organizing the findings by the research questions presents a logical format because each of the research questions involves the CDO and each of the questions build upon each other. This format takes the reader from role of the $\mathrm{CDO}$ in institutionalizing diversity and inclusion, to specific strategies the $\mathrm{CDO}$ and the campus is engaged in "to institutionalize a new way of doing the work" (Kezar, 2007, p.415).

\section{CDO Participants}

It is important to begin with a discussion of the organizational characteristics and qualifications of each of the CDOs in this study because it sets the context for how the CDOs institutionalize diversity and inclusion on their campuses. Each of the CDOs at each of the case sites were not the first on the job at their institutions. This is important because it indicates that the campuses had some prior experience and history of having a $\mathrm{CDO}$ at the institution. For example, at Francis University the $\mathrm{CDO}$ was the second person to take the position at the university. The first person was interim from the faculty until a national search could be conducted and a permanent hire made. The permanent 
hire was the CDO interviewed for this study and has been in her position for 12 years. At Morrill University the CDO in the study was the third CDO at the institution. She began her tenure as the CDO in 2009. The first two candidates were external to Morrill University and each came to the university with administrative and faculty experience at institutions other than Morrill. At Central University the CDO was interim and had been in the job for less than a year. Prior to his arrival the previous CDO had been on the job for five years. The $\mathrm{CDO}$ at this site stepped into the interim $\mathrm{CDO}$ role as a tenured member of the faculty and as a partner in many of the previous CDO's initiatives with faculty. During the interview he expressed his interest in being a candidate for the permanent replacement.

All of the CDOs in the study had previous careers as faculty and attributed this status as contributing to their ability to perform their jobs successfully at their institutions. The CDOs at Morrill University and Central University were faculty from these two universities. The CDO at Francis University had never been a member of the faculty at Francis but had a long career as a faculty member at three previous universities and held administrative positions in Academic Affairs. She described her campus environment in this manner:

I was a member of the faculty at another university before I was hired and brought here. So I do have faculty status. ... [It's] exceptionally important, because faculty tend to dismiss anybody outside of the faculty, including administrators. If they see you as a sheer administrator with no faculty background, then they really don't pay much attention to you; you don't have any credibility.

A faculty colleague at Francis had this to say about the CDO being an "academic:"

She is very well received. ... But being that she is an academic, she speaks the language, she uses...hard data, good theory. In terms of furthering a lot of the goals and objectives it is a very, I think, winning approach. It has been very 
successful. It definitely provides her with a lot of rapport and the ability to interface with faculty.

The CDO at Morrill University had this to say about being a member of the faculty:

It would be hard for somebody to be in this role at a place like [Morrill University] if you didn't come from the faculty. I mean, that's not a model that operates at other institutions but I think that this particular campus, given this particular culture, that makes sense. Other universities don't care whether the person is from the faculty or not. For example, I can enter into spaces and conversations - talking about some key issues relative to search committees for faculty, promotion and tenure issues, curriculum issues - where I think it would be easier having gone through the ranks myself and having an understanding of that. For example, promotion and tenure dossiers come to the provost level to be read before they're voting on to award somebody tenure and/or promotion. I'm one of those readers, as well as others, that the provost assigns. And she assigns readers who are individuals who hold tenure, like myself. So I see that. So, I mean, I'm not arguing for one or the other; I just think it depends on context and depends on culture. The model of the staff person, at other places may work very well. But I think for [Morrill], the model that we've started off with is that the person must have faculty rank.

The CDO at Central who is interim is excited about the possibility of assuming

the role permanently and believes his faculty status give him creditability:

I'm here because of some of the things I described earlier, like there is enormous potential, and so that keeps me excited about the provocative possibilities. The other piece is: people know me. I've had relationships; I've been in community and other spaces outside of my department. And so there's a certain familiarity that I think that I can capitalize on. And knowing that people respect my research enterprise - they may not know a lot about it, but I am a faculty member and my research has been high.

Furthermore, the CDOs in the study were all housed in Academic Affairs. Their titles ranged from Vice President for Intercultural Affairs, Vice President and Associate Provost for Diversity, and Vice Provost for Diversity and Climate. At Francis University the CDO reports to the President of the University but has a close collaborative relationship with the Provost that involves weekly meetings. Her office was physically 
located in the Provost's suite with the other academic administrative officers. She had

this to say about the importance of her physical and organizational location:

Some people don't bother to take the time to understand the value of Business Affairs or Student Affairs, or anything other than Academic Affairs, which is unfortunate. And that's just the nature of higher education. It's really unfortunate. So, it's very important for this position to not only be housed in Academic Affairs, but to act like it's an academic piece.

A member of the faculty at Francis also understood the deliberate choice of the location

in Academic Affairs. He shared:

The office of [the $\mathrm{CDO}$ ] definitely has an agenda to...make sure that the academic side - or academics are as much a part of the process of transformative change as people in student affairs. On many college campuses, people in student affairs generally tend to be doing a lot of the front-line work in terms of student recruitment and mission and trying to inculcate in students or socialize our students to be citizens of the world and have a broader vision of, perhaps, reality. And this has been historically something that people in student affairs have been much more comfortable with, and they've done a lot more. But the work having the Office of [the $\mathrm{CDO}$ ] on the academic side definitely helps to make clear that we [faculty] are stakeholders, and that we're very much interested in diversity work. And also, initiatives that translate into the classroom and the learning experience.

At Morrill University the CDO reports to the Provost but has a dotted line to the

President. She described this arrangement in the following manner:

I chose to report directly to the provost - I have a dotted line to the president; I sit in both cabinet meetings. So I have their ears all the time, as well as my fellow colleagues who are vice presidents.

At Central University the CDO works collaboratively with his fellow Vice Provosts that include the Vice Provost positions over Faculty and Staff, Students, Enrollment Management, and Teaching and Learning. He described the organizational structure as beneficial to transmitting diversity objectives and goals across each administrative unit in a highly decentralized university. In regards to this he shared, "so, yes, we're 
decentralized, but we have to operate or make some kind of decisions with a hub-like structure so we're all on the same page." The Provost at Central also addressed this point:

It's more than just [the CDO's] office. I mean it's the whole Provost shop. It's really important. ... It's highly collaborative. ... It's part of each of their roles and responsibilities. Now, [the $\mathrm{CDO}$ ] takes main hold, obviously. But the teaching and learning - [diversity's] got to be in teaching and learning, it's got to be in faculty and staff, employment issues, and training of faculty, mentoring, all them.

The physical and organizational locations, as well as the reporting lines are significant for the CDO because they signal to the wider campus community the importance of their work.

Related to their physical and organizational location, the CDOs at Francis University and Morrill University described their offices as operating within the Collaborative Model, which is noted in chapter two and refers to Damon William's (2013) vertical structuring of the CDO position. Both CDOs had relatively small offices. The CDO at Morrill University has a research associate and an associate vice president/chief of staff under her leadership. She also has an administrative coordinator and a quarter of a faculty member who leads a difficult dialogue and mediation series. At Francis University the $\mathrm{CDO}$ has a research associate, senior research analyst, and an administrative support person under her leadership. The CDO at Central University had the largest functioning area, which could best be described as Williams' (2013) Portfolio Divisional Model. Several diversity offices for students and staff fell under his leadership. An assistant vice president for diversity and climate, a special assistant, and administrative coordinator also supported him. Noting the different vertical structuring of each CDO functional area is important because it demonstrates possible options to transform the campus. A large university, like Morrill University with 50,000 students 
does not have to mean a Portfolio Divisional based model. Similarly, reporting to the President directly as is the case at Francis University does not have to mean a Portfolio Divisional Model. As this study indicates, the institutionalization of diversity can occur with either the Portfolio Divisional or Collaborative models.

Furthermore, the $\mathrm{CDO}$ at Francis University stressed her preference for a smaller organizational construct for two reasons. First she explained that having a smaller diversity office signaled that diversity was not the job of just one division, but rather was the work of the entire organization. She felt that if the office were small, others would see that not one single person could do this work alone; it would require collaboration. Second, she felt that the small organizational construct was less disruptive to the organization because existing offices and departments that addressed diversity or equity could be left in their respective administrative areas versus moving them under her leadership. At Central University the division lead by the CDO had undergone considerable organizational restructuring over the last six years, which did cause disruption. Departments and offices had been moved from Student Affairs into the CDO's broader organizational division. This caused some disturbances among the staff and required the $\mathrm{CDO}$ to lead a transition plan to get the newly moved units acclimated to different processes in the diversity division.

Another characteristic worthy of noting is that each of the case study institutions had a diversity plan in place that the CDOs in the study was charged with implementing and carrying out. This is worth noting because it underscores the fact that the CDOs were not operating on their own without some type of organizational direction, but rather were operating with a guide and agenda that applied to the entire campus. For example, 
Francis University is operating off a diversity plan spanning the years $2008-13$ and is revising its plan for the next five-year period. Morrill University is utilizing a plan developed in 2006 that was revised in 2009 after the CDO in the study came onboard. It has been updated with timelines for climate studies and assessments through 2015 .

Central University had the longest history of the three institutions in diversity plans. The first diversity plan for the campus was a ten-year plan developed in 1988. During the time of the study, Central University was in the process of creating a new diversity plan for the campus to follow. Previous to this effort, the campus' last diversity plan ended in 2008 and a close out report was issued to the campus in 2009. From 2008 on, the campus utilized a diversity plan devised by the statewide university system. They also used goals outlined by Central University's campus strategic plan. This period in the campus' history was also marked with significant organizational transitions in several diversity offices, which was not conducive for launching a new diversity strategic plan. A more detailed discussion of the campuses' diversity plans as a key strategy for institutionalizing diversity and inclusion will be discussed in the findings related to research question three.

\section{Role of the CDOs in Institutionalizing Diversity and Inclusion}

Returning to the first research question, what is the role CDOs play in institutionalizing diversity and inclusion, the data indicated that the role CDOs play in institutionalizing diversity and inclusion is to lead change. As evidenced in this study, their role is to bring the wider campus along in its efforts to build inclusive and diverse universities in a variety of intentional ways. Furthermore, to lead change the CDOs in the study carry the message of diversity and inclusion to all corners of the university so 
diversity and inclusion can permeate the wider organization and result in changed attitudes and behaviors that support diversity and inclusion. To have this broad effect, each CDO in the study worked at the highest levels in their respective universities to effect change and served as a centralizing resource for all constituents. One CDO described her role in this manner:

My job is to be in every place possible to impact policy and procedure; I work at the very top...of the institution to promote change. For example, I'm in the President's Cabinet, I'm in the Dean's Council, I'm in the Provost's Planning Council, I'm a member of the Provost's Council, Enrollment Management Council - all the top-level pieces in the university that make policy - that's where I am. ... and as part of the President's Cabinet, I go to all the Trustee's meetings and all the Regent's meetings.

At Morrill University the CDO "is hired to own the campus' strategic diversity plan" and takes responsibility to be the campus' "voice" in this effort. From this plan Morrill University's entire campus' diversity operation is laid out and the role of the CDO in her words is to,

first and foremost ... assist the President and the Provost to hold the university accountable for our diversity efforts. That's my primary role. And, of course, other things that come with that. I not only work with the Provost and the President, I work with my fellow vice presidents, I work with deans, I work with department heads, to make sure that whatever we're doing in terms of diversity with our efforts, that we have clear assessment measures in terms of measuring our progress.

At Central University the $\mathrm{CDO}$ is considered to be the centralizing point-person for several campus diversity efforts and strategies. A campus partner working closely with the CDO described their role as "very critical because you have these locally based efforts but we need some kind of centralizing, coordinating mechanism. And that is what the Chief Diversity Officer does." She goes on to state that the CDO provides, "visibility for these efforts and that is something that has to be done centrally. You cannot do it 
locally, and individually - there's got to be clear leadership, and that's what the CDO provides."

CDOs efforts to construct new attitudes and behaviors and institutionalize diversity and inclusion are evident in two themes that consistently emerged from the data. The first theme to emerge is CDOs operate with intentional strategies that require relationship building when working with campus partners. Although all the CDOs in the study have high levels of positional authority, they work deliberately to build relationships with campus partners to influence change rather than mandating it as senior leaders in a hierarchical schema. This is important because it speaks to the intentional relationships that have to be built despite high-level titles or proximity to the president and provost. In these scenarios CDOs customize their approaches to meet campus partners "where they are at" in the change process by "working alongside" them.

The second theme that emerged from the data indicated that institutionalizing diversity and inclusion takes time. CDOs and campus partners were very much aware of this condition as they engaged in the change process. Throughout the study participants referred to the change process in terms of "then" and "now," referring specifically to the sense of time involved. In addition, several participants indicated that there is perpetuity to the work. They shared an understanding that the work is never really done. A detailed discussion of each theme related to research question one; the role of the CDO in institutionalizing diversity and inclusion follows.

\section{Relationships and Intentional Actions}

The three CDOs in the study operate with intentionality to achieve specific goals and outcomes. As a result, their work is based upon relationships and relationship 
building that result in buy-in for the campuses' diversity agendas. Often times CDOs are considered partners and advocates rather than authority figures mandating change from the "top-down." CDOs customize their approaches to meet campus partners where they are at (either early, mid, or full transformation) in the change process, which may vary from group to group, department to department, and administrative unit to administrative unit. At Francis University the CDO was tasked with improving faculty diversity after the campus was making little improvement in this area. To address this concern it was determined by the President that the CDO would actively be involved in faculty hiring. This resulted in departments having to demonstrate to her and the Provost how they were going to recruit diverse candidates before permission to hire could be authorized. Working intentionally, she devised trainings and manuals to help faculty recruit minority candidates. She worked one-on-one with departments to produce job announcements attractive to underrepresented candidates. She also spent time discussing data and research on personal biases and how they can result in homogenous departments, which limit student learning. The CDO explained that thinking along the lines of diversity and equity were not always evident in faculty hiring but she spent concerted time and attention in this single area to get buy-in and eventual support from department faculty. She reports:

When we first started it, they fought like the devil. They didn't want anything to do with the training, because their thing was, 'Look, we've done this, we've been hiring for years, and we've been doing a good job.' But they were hiring all white men...I work all over the university with all the search committee chairs as they are hiring, and we provide training for them - before they actually begin...our hiring process. 
To facilitate this process this $\mathrm{CDO}$ conducts the trainings herself and provides hands-on assistance with the faculty and staff who need her help. Her office provides reports on the historical trends of hiring diverse faculty and staff in individual departments and units. Based on these reports, departments with low numbers of diverse employees receive more of her time and attention. When asked what attributed to this shift of wider acceptance she reported,

a President who supported it and faculty who participated in the development of the document, the training manual. See, if they can participate - from a research point of view-when you are developing a document, and they know that it's research-oriented, they don't push back as much. And you'll find somebody in the faculty who will begin to acknowledge what it is you're doing.

A member of the faculty at Francis University who has participated in these trainings confirmed the CDO's approach:

I think one of the main approaches that has been very successful here on campus - and much of it is to the credit of [the CDO]-- is her approach tends to be to build relationships -- interpersonal relationships across the campus. And through those relationships we began to up-front our values as far as diversity and inclusiveness. And by also trying to get members of the entire community to buy-in.

Similarly, the CDO at Morrill University also accredited much of her and the campus' success to intentional relationship building. After a four-year review, she realized that much of the campus' success would rely on her ability to cultivate trust and acceptance among current and future supporters. She shared,

I worked very hard at this intentionally - to build relationship on campus; to really understand people where they are in order to get them moving and in order to get buy-in. So that's been very important to me. So yes, I think building relationships is a huge piece of this job.

This strategy proved useful as she worked to advance a newer version of the campus's diversity plan that she had modified to gain stronger accountability across administrative 
and academic units. According to her, previous attempts at advancing the plan were delivered as a top-down mandate, which negatively impacted support. She described it in this manner:

Before we said to the university community, 'This is a plan, and we're following it.' I convened a lot of stakeholder conversations on campus to get their feedback. So, starting with our Assistant General Counsel, student leaders, faculty, staff organizations, department heads; I met individually with all the deans, I met individually with all the department heads to get feedback on the diversity plan. So by the time we were ready to roll it out, a lot of people were already on board, had given their input, so we were ready to go.

For her, gaining support in advance helped with the role out and adoption of the current plan, which she credits to greater acceptance and improvement. To continue building support she utilized a coaching technique she described as "praising and challenging." According to her,

praise them when in their mind they might think they have made a big step. In my mind it may not be a huge step but, in their context, it's a big step so saying, 'Wow. That's really good. Keep doing what your doing.' And at the same time, when I give them that little carrot and say, 'Okay, you're doing well here; remember this is what I would like you to work on for the future.'

The CDO at Central University described a similar approach when balancing topdown administrative initiatives and partnerships he needed to develop. Drawing upon his theater background, he refers to it as an "ensemble effort."

The collaborative process is how you make theater - that's what I'm trying to bring to this realm. Trying to bring a spirit of collaboration that acknowledges your expertise in your area. So you're my costume designer, you're my scenic designer....Everyone has an important role to play, but we're all focusing on the same goal.

For him, building relationships with faculty became essential in order to get buy-in for the campus' diversity and inclusion efforts and to build trust. He shared that his style of leadership is not about "naming, shaming, and blaming." Rather, he frames his work 
with campus partners as "putting our best foot forward and creating that safe space" to do the work. To have those "tough conversations" with faculty and other academic leaders, the $\mathrm{CDO}$ at Central University likes to create spaces to "challenge assumptions" and find solutions together. To do this he makes sure that he and his office are in partnership with the Dean's of the Colleges and Schools at Central University. He stated,

and if your Dean is in community and partnership with the person who sits in this seat - and that is something that I'm constantly trying to do, is build those relationships - how can we be a support mechanism for you [the Dean] rather than a policing mechanism? Because I think that's the key. Many people have a whole host of responses when we think about our diversity, our cultural competency training, and anything that's quote-unquote 'required' - well good luck doing that here in Central.

He shared that conversations, relationships, and clear communications that outline the "incentives and disincentives" help. Each of these conversations are customized to fit the specific needs of the department he is trying to assist.

Participants in the study who work closely with the CDO expressed appreciation for these relationships and partnerships. One administrator from Morrill University described the CDO as an "advocate" who advances issues they have been working on in his division. His unit is responsible for undergraduate and graduate admissions. He shared that the CDO had requested a study on why African-American and Latino students were turning down offers of admission to Morrill University. As the Assistant Vice President for this area, he and his team were well aware of some of the most prevalent reasons, but the report commissioned by the $\mathrm{CDO}$ helped with validating these observations. Furthermore, the $\mathrm{CDO}$ presented the findings from the survey to departments who could help address challenging areas. In this case the focus was mainly on the office of Development who could help with scholarship support and the Alumni 
Association who could help with local recruitment. He shared this comment about the

CDO:

She [the CDO] presented to, basically, I think all of those constituents, including Development. So she really has put the challenge out there that, if we're going to be competitive and getting these students to enroll, the packages need to be there....So I really feel that she has done a really good job of getting that report in front of everybody. And she's gotten the administration on board; the Provost is on board and then, certainly when we get a new permanent President, I know he or she will get on board as well.

Regarding support for alumni volunteer recruiters he also shared:

When [the CDO] came on board, she really worked with my Director of Recruitment to sort of formalize these programs. And so the last two to three years, these [actions] have become more formalized, more organized and I think, therefore, more effective.

For him this was significant because the CDO was able to take over in organizing the alumni rather than his office that had other priorities to work on simultaneously.

Similarly, another administrator at Morrill University in a different department credited the CDO's intentional actions for bringing speakers to campus for staff trainings and dialogues. Previously departments struggled on their own for staff development and training, but he credited the $\mathrm{CDO}$ for advocating that this be a priority that divisional leaders adopt. He shared, "I think the visibility of [the CDO]...you know, she's brought in speakers, so the conversation around diversity is happening at different levels at the institution with her speaker series." He credited this support as making it easier to lead conversations about the importance and role of diversity in his own department. He was thankful that through the intentional actions of the $\mathrm{CDO}$, more awareness about diversity and inclusion was occurring campus wide. This legitimized his department's efforts to promote diversity and inclusion in his division.

Sense of Time: Long Trajectory 
As change leaders, all of the CDOs and incidentally each of the Provosts in this study acknowledged institutionalizing diversity and inclusion takes time and often occurs incrementally with quicker successes in certain areas and slower progress in others. This acceptance was coupled with the belief that although institutionalization of diversity and inclusion has occurred, there is, and always will be, "more work to be done." For example, in a discussion about larger faculty participation in cultural competency training, the $\mathrm{CDO}$ at Central University indicated that his efforts would take time. Although being an immediate priority to the campus, he realized that,

it's certainly going to be something that probably won't bear fruit for a couple of years, but I think with this program, just as the learning communities and the [Central University] training methodology, many of those other partnerships that I talked about earlier took time but faculty were eventually present.

The CDO at Francis University who has been in her position for 12 years, shared that she did not see the fruits of her labor for four years. When asked if departments were now more accepting of the practice of assessment that she initiated for diversity reporting, she shared that it is now "endemic to what Francis University does." However, this was not always the case; as she shared, "when I first came here and started talking about data, they wanted to shoot me." Yet, she stated, "Francis University has responded to the institutionalization [of diversity and inclusion] over the years in a very positive way." She stressed that, "it took a good four years" before attitudes started to shift and the practice was accepted. She credits these changes to the "evolution of the organization" over time, which can also be described as an eventual paradigm shift.

A participant in the study also engaged in diversity work at Francis shared that although certain improvements had been made, complete transformation would still take 
more time. He was somewhat comforted by the fact that they were not the only

university faced with challenges in equity. He reported:

We would hope that the gap is being closed, that the disparities will be minimal. But we realize that this is a national trend. It's not just something that's unique to Francis University, in terms of the academic discourse about academic units and the work that is essential in terms of diversity. But I think we're making progress. There's an openness. It's no longer a novel conversation. It is a conversation that people are having on some regular basis. I think the whole concept of diversity and inclusiveness is very much a part of the everyday conversation. And it's a concept that people are increasingly familiar with, comfortable with, and we hope that it will continue to translate in terms of impacting the campus life. But in terms of how long, I really don't know. We're hopeful that we'll continue to make headway and have gains.

At Central University a graduate student who is a member of the campus' ad hoc committee charged with writing the next diversity plan shared that he has had difficult conversations with fellow students concerning the lengthy process involved in changing the culture of the institution. Expressing his frustration regarding the time involved in affecting change, he shared these comments:

We're talking about changing the way things are done here, and changing the culture, to change the climate that will keep the people ... we have actually here, so that they start to ... attract more people to the institution. They don't want to hear any of that, because I was saying, 'This is not something that I will ever see, you will ever see, any student who is a freshman for the next ten-fifteen years will ever see the actual fruits of this labor. It's going to be a very long process.'

The Provost at Morrill University also discussed the time it takes to accomplish this sort of change, describing it as a "sequence of pressures" that has brought about incremental change at her institution. However, she is okay with the slowness of it because then individuals can act with more purpose and intentionality. In regards to annual accountability reports directed at her office she shared:

Because they believe we're going to do this over and over again, they don't have to be in a hurry to show something in one year; I say, 'just make something that will [succeed] in your college or your division and go forward.' 
For her, it was more important that departments and divisions carefully think about what they are trying to accomplish to get at deep changer, rather than simply trying to comply with an exercise. She reiterated, that this was not a "onetime" exercise, but rather an "ongoing practice" for transformational change.

This sense of change over a long trajectory was coupled with a strong belief that there is always a need for ongoing attention to diversity and inclusion efforts. The Provost at Francis University articulated this best. He shared:

Well, it's interesting because I think that one of the characteristics is that we're never satisfied. And so I'm glad that we are identified as a university that people look to as doing it well. And part of, I think, doing it well is never feeling like ...you're doing it well enough.... Well, I do think in some ways the challenge I fear most is complacency. Especially if you - as you say, you've come in - and I hear this from others, too - is that Francis University is thought of as a place that's doing certain things pretty well.... I don't think we're in danger of it at the moment, but I do think that sometimes the biggest impediment to being truly outstanding is already being pretty good.

For him and the people he directs, he is comforted by the fact that through the efforts of the $\mathrm{CDO}$ individuals are constantly being challenged to improve. For him those who believe they have done a perfect job will eventually believe "I don't have to keep working at it" and for him that is not an option for the university.

The CDO at Central University, who shared that his campus has been successful at institutionalizing diversity in certain in areas, recognized that there are still areas that need work. Concerning the structural diversity of the undergraduate population, he reported that there is a "groundswell, but when you look at our overall population of 40,000 students...there's still a lot of room to grow." Another administrator at Central University who works in Academic Affairs also stressed this point. Regarding 
improvements she has witnessed throughout her career in outreach and access, she shared:

Now you see people who are involved in these efforts, either they are initiating something or they are checking with others, seeing how they could do some collaborative kinds of things. And so it's really something that is campus-wide. It has been integrated into the culture and fabric of the institution. Do we need to do more? Of course we do. Hopefully, there will be a day - but I probably won't be alive - when diversity is just a way of life.

Similarly, the Assistant Vice President for Student Affairs at Morrill State University commented,

I think we have...we've made some tremendous strides since we had our first chief diversity officer. ... So, are we reaching our goals? Definitely. Is it institutionalized? Yes, it is but there's still work that needs to be done. So we're there, but we still have work to do.

In summary the way in which CDOs lead change to institutionalize diversity and inclusion, involves intentional actions that are aided and strengthened by concerted relationship building. Through intentional actions and relationship building, the CDOs in this study have greater ability to carry the message of diversity and inclusion so that attitudes and behaviors toward diversity and inclusion change in a positive direction. Furthermore these intentional actions and relationships ensure a deeper permeation of the change effort. Through time and ongoing efforts, these new attitudes and behaviors become more permanent to the organization and are eventually normed. However, as the data suggests, constant attention and upkeep are needed to sustain the change effort. This requires ongoing maintenance led by the $\mathrm{CDO}$ to keep the institutionalization effort permanent, especially as new students, faculty, and staff enter the organizational system.

Evidence of Institutionalization Efforts 
Research question two asked, how do the three case study institutions know they have institutionalized diversity; what are the outcome measures and who is involved? Data from all three campuses in the study indicated that the institutionalization of diversity is evident when attitudes and behaviors surrounding diversity and inclusion shift from traditional notions of diversity to a broader understanding of the complexity of diversity and its benefits to the university. As part of this broader more complex understanding the universities in the study gave additional attention to campus climate because it relates to how welcoming the campus is to diverse people. Thus with their more complex understanding of diversity, the campuses recognized that diversity efforts also had to address inclusion. The outcome measures the campuses used to determine their institutionalization efforts varied between the case study sites and were not uniform throughout each campus. The CDOs in each case study site worked with the units within their institutions to come up with appropriate outcome measures based on institutional data the CDOs analyzed and assessments they conducted. A detailed discussion of the findings related to the knowledge of institutionalization of diversity efforts and outcome measures follows.

\section{Broader Understanding of Diversity}

In order to move beyond "diversity for the sake of diversity" or diversity as an exercise about "counting numbers," as the Provost at Morrill University described it, the three case sites worked to transform people's attitudes to think differently about diversity, particularly beyond racial diversity. This is important because it moves beyond older models where diversity is thought of as an extension of affirmative action or compliance 
with federal regulations. In the study the three Provosts emphasized this point

specifically. The Provost at Francis University explained:

You have to fundamentally...ask, why do you care about diversity? This is not thirty years ago, when you cared about diversity somehow because you wanted proportional representation. What does [diversity] bring to the community? What's the richness of the tapestry that you create when you have people with all kinds of different backgrounds, different experiences?

He went on to share that at Francis University,

diversity isn't something over there to be achieved, and it's not something about numbers and tables - not that you don't look at those things, but you have some sense of why are we a better community and why is our education a better education for our students.

This broader approach to understanding diversity is fundamental to Francis University, which under the leadership of the CDO uses an intercultural model to inform its diversity efforts. According to the CDO "interculturalism is a step above diversity, because what it means is that people - a diverse group of individuals - are working together to understand each other." She admitted, that when she first arrived at Francis University she spent most of her time explaining to individuals, "diversity has nothing to do with affirmative action," but overtime people were willing to embrace this new construct.

The Provost at Central University described the gradual shifting of attitudes toward diversity as core to his institution's values. He shared:

I think it goes back a very long way. [At Central], the campus has always taken this very broad attitude, holistically, about diversity. And let me expand on that a little bit. I don't mean just racial diversity. I mean diversity in every sense of the word. Kids coming to our institution from [Small Town, State], population, 20, class size, 4 . And we view that as diversity in the same kind of sense.

He also shared that making the argument for diversity solely a numbers game made him uncomfortable and defeated the purpose of truly transforming the organization, or the "environment." He shared, 
I've always been resistant to putting numbers associated with something. Because when you put explicit targets, then you tend to do that activity for the purpose of reaching the target. Which is not all bad, but that doesn't really change the environment as much. So that's a much harder thing to define: what's the environment we want at Central.

At Morrill University, the Provost explained that they were focused on a more intentional shift in order to get her administrative team thinking differently about diversity, particularly in how they measure diversity.

It's about the culture of the organization...And it was really easy for everybody to think diversity was about how many 18 -year-olds we got in who are AfricanAmerican or Hispanic, and you're like, 'That's really not it.' And so it was easy for us to make that decision about what it means to have a culture that is getting much smarter and better about being a diverse organization, and a learning organization. ...We have gotten past just having certain policies in place, and rules in place. We've gotten beyond - not past - counting numbers to see this and that.

To give an example of how she was challenging her staff to think more broadly to create a cultural shift on campus she shared:

Praise whoever we could get in here where we thought we had enough AfricanAmerican and Hispanics. Then would we be done? Maybe, maybe not? It depends on other...things. I mean, Muslims haven't found it very friendly here, you know? People assume because we're nice and friendly - we say 'yes ma'am' and all this - we're friendly to everybody. And so I think the more we keep learning about ourselves is important. People buy into that now.

In order to achieve an acceptance of a broader definition and understanding of diversity, the three institutions in the study recognized that notions of diversity had to also involve majority groups, which at all three institutions were White. This is important because the CDOs in the study included the diversity and climate issues of White and majority students in their understanding of diversity. For them solely focusing on people of color or minority groups did not espouse the broader understanding of diversity and inclusion they were striving for at their institutions or in the 
institutionalization effort. At Central University the CDO shared that diversity work also includes his majority White students. When asked if one of his goals was to get more non-persons of color or marginalized groups to participate in cultural competency trainings, he shared,

that's the only way it can work. When you look at our population, students of color on average - roughly 14.8 to $15.3 \%$ - you still have a healthy chunk of students that are majority students, some from very affluent backgrounds; some from rural communities that are coming from very impoverished backgrounds, as well. And yet, their experiences are kind of lumped in this big category as 'majority.'

To ignore their stories, he indicated it would be a mistake and to dismiss them completely because they are from racial majority groups as this would limit the notion of diversity at Central. At Francis University, the CDO stressed that she is most effective at her job when she has broad reach, including serving White populations.

I don't focus just on people of color because once you start doing that then the White people dismiss you. You have to focus on their issues, too. And you have to help them understand, many of their issues are the same as people of color issues.

She went on to say that she would not want an office whose sole focus is "minority issues."

I wouldn't want an office like that. Not in this day and time. I think that my office has credibility across the institution because people see it as an inclusive place and that it focuses on inclusion, so they feel comfortable coming regardless of the color of their skin or their ethnicity....But the point is, that as many White people come in this office as people of color.

From a programmatic perspective, the department for intercultural student services at Francis University readjusted it's focus to be more inclusive to White students and students from mixed races as well. They created a special office alongside the 
traditional "ethnic offices" to welcome students from these backgrounds and to focus on their specific needs. The director of this center shared that the new office,

has been charged with multi-race, White students, those students who most likely will be, like, 'I don't think that I fit in with Chicano/Latino students, or Black students.' They might come to things, but how are we addressing their needs?

Emphasizing the breadth of diversity, she described her efforts and the evolution of her

department in this manner,

It has really truly moved from just concentrating on students of color to really engaging the rest of the campus. So even though we haven't necessarily moved away from having ethnic offices that addresses their particular needs, that we are open to have the rest of the campus to be part of that conversation. ... without this... we couldn't say with integrity that we are encouraging the development of the whole person. So we come in as one of those departments that can really help the students learn about themselves, learn about others, and learn about those exchanges.

Morrill University also directed efforts to broaden concepts of diversity to examine the needs of women and the LGBT community. In the division of Student Affairs, staff were addressing the problem of why so few women students take up leadership roles. The Assistant Vice President was asking his staff, "how inclusive are our organizations? How inclusive is the leadership in our organizations?" He attributed some of these challenges to the institution's strong military roots that date back 125 years. Regarding this trend, he challenged his staff to expand their notions of diversity and to address this gender discrepancy in student leadership as a diversity problem. $\mathrm{He}$ stated,

We have concerns about the women that are here...The majority of our premier organizations are headed by men, and the women are very, very capable here. ... So I don't think it's pushed, but I think its just part of the culture that 'these are positions for men.' We've noticed that our Student Government Association, we've had all males. We haven't had hardly any females even express interest....So, that's bringing in a whole other dynamic of diversity, but it's from the gender side. So how inclusive are we and what are we doing with our 
advisors to ask these students these questions?

Also at Morrill University, a group of LGBT students expressed concern to the Provost that they felt unsupported on campus, particularly by their fellow students. They aligned themselves with liberal-minded faculty and wanted to become part of the CDO's office in the Provost's purview. This would mean moving the student organization from Student Affairs to Academic Affairs. The Provost pushed back and shared that in order to remedy the situation or any perceived problem, Student Affairs needed to be involved. To move the student group into a different area would send the message that "diversity" is the work of some and not the work of the collective organization. She needed to "hold all units accountable" and treat this as a diversity opportunity. Rather, she preferred that more concerted attention be delivered by Student Affairs to meet the needs of LGBT students. This involved expanding the notion of diversity to include LGBT students and their wellbeing at the conservatively oriented campus.

Through the leadership of the CDO and support by the Provosts, the need for change was also framed around the goal of producing more diversity and diversityminded graduates. At Central University the CDO used this more expansive argument regarding the importance of diversity and inclusion to justify resources targeted at programs that support historically underrepresented minority students:

Some people may see it as we're giving handouts to these students, or we're spending way too much money to try and attract a very small population. When, in reality, what we're saying is the skill-set that our students are going to need for the 21 st century - if they're going to be marketable and competitive - they've got to know how to interact with students from all walks of life. They've got to know how to interact with students from different sexual orientations or backgrounds. We have to expose them to that. That is as fundamental as understanding the basics of calculus. So it can't all be about math and science, right? 
The Provost expressed a need for a more diverse campus to produce graduates ready for the world beyond Central:

There's no question that diversity in the more classic sense is very important for us. Mostly, because we have to educate students to participate in a world environment where it's not going to be a bunch of Norwegians from the [Midwest]. Right? It's going to be a very broad spectrum of society, and they need to have an understanding of that broad spectrum of society. And what the cultural norms are outside the university.

He takes his cue from meetings with the state legislator and private industry. According to him both are asking for graduates with cultural competencies beyond their own identities that can meet new demands of the global economy. He shared:

And when the industries in the state tell the state legislature that [Central University] is training the kind of kids that we want because of these diversity elements, that's a high impact. I mean if you're at GE, and you're in the medical systems group, you're a worldwide market. They want people who speak two languages. They're not caring very much which languages, but they like Mandarin-speakers and Spanish-speakers. They want people that they can place in different parts of the world, who are culturally knowledgeable about those parts of the world. It makes a huge difference. You don't have to be a native [of that country]. You just need to understand.

A diversity administrator at Central University who worked at the university's school of medicine credited pressures from outside medical organizations in the early 1990 s to get the school to think beyond diversity as affirmative action. Groups such as the Association of American Medical Colleges produced guidelines to help schools produce diverse and culturally competent medical students that could work in several medical markets including rural areas and the developing world. This argument is one that continues to shape how the medical school engages in diversity work at Central University. In working with faculty to make this transformational change toward diversity this administrator continues to see this argument as helpful in discussion with those still pondering the need. Rather than looking at this approach as helping "one or 
two individuals" she challenged faculty to think "how it could benefit not only the school and the state, but society as a whole, and really assist in health care issues" plaguing the community. The CDO at Central agreed with this argument and used it in his conversations with faculty and campus leaders.

\section{Campus Climate}

In addition to changing attitudes and behaviors to yield broader notions of diversity, the campuses in the study acknowledged that improvement in campus climate for all stakeholder groups (students, faculty, and staff) was equally important to know if the institutionalization of diversity and inclusion was occurring. As such, the CDOs in this study took deliberate steps to examine campus climate separately in order to ensure strategic diversity efforts were truly making an impact.

At Morrill University the climate for African-American and Latino students was of particular concern to the $\mathrm{CDO}$ and other administrators interviewed in the study. Three administrators within the case attributed the less welcoming climate to its location in the South in a rural conservative oriented area. The Assistant Vice President for Student Affairs pointed out that the most recent campus climate survey for students indicated that African-American students reported the lowest sense of belonging.

There are still some conversations being had around different tables about: 'This place is not friendly' or 'We're too conservative'. And I think our students, when they get here, they appreciate the traditions, they appreciate the education that they're going to get, but I think it becomes a reality about where they are physically.

He also reported that the most recent student climate survey revealed that underrepresented students felt "like they had to give up a piece of their identity in order to fit in here, and that shouldn't be the case." He shared: 
And we asked the question: where are you feeling the most stress? They feel the most stress when they are around their classmates. They feel the least stress in their student organization. Well, in their student organizations they're selfsegregating, so they're going into places where they can let their hair down and feel comfortable, okay. But when they have to do that project with their classmates, they're having to somewhat code-switch and be someone else for a while.

For him, understanding the climate data is critical for he and his colleagues. They use the information from the campus climate assessments to help students feel more welcomed at Morrill University and addresses issues about inclusion. He also uses it to train his colleagues about what students are truly experiencing and how it varies among different racial and cultural groups.

The CDO at Morrill agreed that campus climate still challenges institutional efforts to be diverse and inclusive.

I think the greatest challenge for us, that requires a lot of our time, is climate issues. Climate issues is very huge, very complex, because you're talking about structural diversity issues, in terms of numbers; you're talking about the behavioral climate, people's attitudes and their beliefs, which is huge.

She also acknowledged that the legacy of the campus presented challenges.

Do we have a legacy of inclusion or exclusion? I mean it was not until 50 years ago when we started admitting women and African-Americans here at the university. We've had a better history with Hispanics than we have with AfricanAmericans. And we just celebrated 50 years of inclusion as a university last year.

The CDO was proud of what these minority populations had achieved at Morrill University in such a relatively short span of time. She was inspired and felt that the future would be more equitable but noted that with greater diversity, campus climate still needs to be addressed and monitored so that all groups feel welcomed and have the ability to thrive equally. 
To address climate issues for students, Morrill University was also looking at how the staff at the university treats its students. According to the Provost, she reported that most often one will look at what is going on in the classroom but sometimes the problem is with the staff. She explained:

Sometimes ... the non-White student felt less welcome by this predominantly White staff than the others. And everybody's acting like: it's the faculty and the students. But the advisors, the Student Affairs professionals ... but the secretary in the front office who let's them in, doesn't let them in. That has to be part of it, as well.

To address this fact, the Provost was working with her own clerical team to address personal biases and asked them to consider how certain sub-groups on campus were being treated. She admitted:

At first all [the staff] could think about was how they felt unappreciated and not valued, which I understand. But now we have them talking and thinking about why certain groups feel even more... like they don't have much of a chance.

At Francis University climate assessments revealed that LGBT students were not doing well at the university. This was problematic to the $\mathrm{CDO}$ because as members of the community their needs were as important as other groups, despite the campuses faith based roots. In this instance, data from the climate survey was very influential to the campus' leadership. The CDO could explicitly detail how the students felt and what could help address issues of isolation and despair. The CDO expressed her concerns:

with students, we didn't have anything formalized for LGBT students. And, again, we found out that the LGBT students were just fledgling - I mean, there was nobody assigned to them, they had no home to go to within the university, they had difficulty finding community. And we used the study to promote the development of a home for those students. And we got it. And Student Affairs was also able to hire an 'out' gay male to be their Director of LGBT students. We'd never had that. But yet, the university - ever since it's been a university has had gay students; gay is not something that's brand new. 
As illustrated in the example above, by assessing how students felt as members of the community, Francis University was better posed to build a more inclusive space for students.

The success of their efforts was reflected in the comments made by two students interviewed at Francis University. The students indicated that they felt that the campus' climate was supportive of their identities. One student identified as Asian Pacific Islander (API) and the other, although he declined to state his ethnicity, shared that he felt the campus was supportive of his identity. At the predominately-White private institution, the API student indicated that coming from an urban public high school with high concentrations of underrepresented students, she was worried. She shared,

My school was predominantly Asian, Blacks, and Mexicans. When I, like, saw that there was a lot of Caucasians I was like, oh. It kind of makes me feel uncomfortable, because I' $m$ not used to being surrounded by all these kind of people.

For her, participation in a summer program geared toward API students was helpful in establishing herself at the campus.

I wanted, like, a part of home....The reason why I chose to go to this school was from the APA overnight, which is where all the Asians get to see the school before they actually attend it. That really helped me because I felt like people wanted me to come here. And then, like, I had people to support me.

The same student also shared that in high school she was the recipient of racial slurs from classmates but this had never occurred to her at Francis University, which surprised her. The second student present in the interview indicated that this was a result of a lot of student lead dialogue about cultural differences and understanding.

The climate of faculty was also important to other participants in the study, particularly to the Provosts and the CDOs. At Francis University a faculty member 
indicated that although levels of faculty diversity are high, when two or three faculty of color leave it is concerning. He reported that in some departments with little representation this could be a significant decrease. For him it was important to know what the climate was like for those individuals and why they left. In these situations, he reported, the data from the faculty climate survey becomes "quite persuasive." The Provost at Francis University reiterated the importance of the campus climate among faculty. He has the CDO interview faculty who are leaving the institution. He shared, "you want to know why people leave. You want to keep track of who leaves and why they leave." As such, he articulated the importance of climate surveys to help the institution to be more welcoming and diverse.

With climate surveys and with interviews of people who leave, who stay, you get clues about what is it - how can you make the institution work better for people. And so how do you find that kind of a welcoming kinship network for people when they come in, that provides kind of a scaffolding that's really helpful....So I think its things like that; there's the basic data that I would think any modern place would collect and report as part of its institutional research data. But then there's the climate surveys, there's interviews with faculty who leave.

According to him data from climate surveys and exit interviews are more informative of "what's really going on" and where the university can address a need.

At Central University the Provost reported, that the university is hiring about eight percent of the faculty population per year, so diversity becomes an important element in each potential search. The Vice Provost for Faculty and the CDO "actively work with the divisional deans, department chairs, and search committees" to ensure that "diversity is a significant element in the hiring process." Furthermore, his office sets aside resources to help departments recruit diverse faculty. If they are successful, he will pay the salary of the faculty person for the first three years as an incentive to the 
department. If the search results in a faculty partner situation that is also a diverse candidate, they may help more. Due to the fact that a considerable amount of resources and effort are put into bringing diverse faculty to Central, the Provost wants to ensure that they stay at Central. This means that the campus climate has to be welcoming and supportive of diversity and inclusion, otherwise faculty may choose to leave. As he explained, "the support for diverse faculty is always tenuous. So you have to constantly...make sure those resources are being used effectively as possible." Through collaborative partnerships with faculty administrators, the CDO works to monitor the climate of faculty.

Campus climate regarding issues of diversity hiring and retention was also a priority for the $\mathrm{CDO}$ at Francis. As she put it, "the university does not have any problems diversifying the faculty any more because faculty seem to now understand the need to have people of all sorts out in front of the student body." However, she admitted that a problem still exists in certain departments helping diverse faculty settle-in. She explained, "you still have a few stragglers where they really need to pick up their feet and take more of an active role in mentoring the new faculty" so that they stay and feel welcomed.

Staff climate in the study was discussed least, but still showed to be an area of focus and concern. The CDO at Central University identified staff climate to be a growth area for the campus; some climate issues were being addressed but more needs to be done in his opinion. He shared that this particular area is challenged by the amount of staff employed and the assortment of positions. For example, any type of programming would have to be split among the different shifts existent in the university, including the late 
night staff crews, known as third-shift. Any type of recognition or training would have to be cognizant of these multiple work hours and the staff who worked the late shifts. $\mathrm{He}$ shared:

So, we have roughly 1,900 staff employees of color....and these are third-shift employees, these are custodians, these are people who are in our dining services, these are people who are also our academic support services. It's a broad slice and... we do certain programming to make those individuals feel supported but it's not nearly enough.

He shared one example to highlight a missed opportunity. The Chancellor and her staff recently hosted an ice-cream social as a gesture of staff appreciation. However, as the CDO noted and subsequently heard complaints about from staff, the hour of the icecream social "did nothing for our third shift employees." The event was held during the noon hour when the third-shift employees were not yet at work. The CDO noted that the majority of the third-shift employees are staff of color and that several as a result of the event, felt under-valued.

At Francis University a climate survey exclusively for staff was administered. According to the $\mathrm{CDO}$, the results of the survey reveled that "staff in certain areas were feeling beat down, ostracized, not cared for." As a result, she put into place a staff climate team, a group of people to review the climate of staff. Now as she describes it:

There are 90 people across the university now working on changing the climate across the institution, in numerous units....Teams in each unit - work with the head of that unit. For example, if it's a college team they work with the dean; if it's a division team they work with the senior vice president. And it's really making an incredible difference. We just had our first meeting where everybody reported out, and people talked about the differences, how they're feeling now. Because they're feeling like they're needed and they're wanted and they're part of this institution, regardless of who they are. This is staff from the groundskeepers all the way up. 
As indicated by the CDO at Central University, because staff workforces can be large and have considerable variety between positions, staff climate can be challenging to assess and analyze. However, the campuses in the study recognized the importance to also look at staff climate in addition to the climate of students and faculty. This is important because it demonstrates that the campuses in the study are taking a holistic approach to applying concepts of diversity and inclusion to staff in addition to the more common habit of focusing on faculty and staff. This also speaks to the breadth in which efforts to institutionalize diversity and inclusion occur.

\section{Outcomes Measures}

All three CDOs acknowledged that accountability and outcome measures are essential to the campuses' diversity and inclusion efforts and contribute to the sustainability of the work. At each campus the approach for establishing, measuring, and reporting outcomes varied. However, all three campuses through the leadership of the CDO relied heavily on institutional data tracking of compositional diversity among campus stakeholder groups (students, faculty, and staff) and data from campus climate assessments. Morrill University had the most elaborate system developed to assess improvement across the institution. Based on support from the President and Provost, and guidance from the CDO and a campus-wide Committee on Climate and Diversity, Morrill University identified three diversity goals for the campus: accountability, climate, and equity. The accountability goal aims to hold individual colleges, schools, and administrative divisions on the campus responsible for diversity and climate efforts. These goals are achieved by rewarding units that are making progress and by working closely with those that are not. Each unit benchmarks itself against an external peer 
institution or national organization. For example, the Provost at Morrill shared that the School of Engineering may compare the diversity of its faculty against a similar Research I peer institution's School of Engineering faculty, or the Athletics Department may compare the diversity of its student athletes or coaches with their national conference peers. Compositional diversity is the measure most utilized. This is broken down into the quantity of diverse individuals and their retention at Morrill University. Biennially, the unit heads, e.g., the Dean of the School of Education or the Vice President of Business Administration gather reports from the departments under their purview and present a collective unit report in person to the Provost, $\mathrm{CDO}$, and the Committee on Diversity and Climate. The efforts of the unit are measured against the previous two-year period and data from their designated external benchmark group.

According to Morrill's diversity plan, the goal areas of climate and equity seek to improve the campus' environment for all individuals at Morrill University and in particular for those groups who experience exclusion or have disproportionate rates as evidenced in climate assessments and institutional data. According to the diversity plan, units are responsible for examining existing policies that may inhibit certain groups from achieving equitable rates in promotion, academic achievement, or graduation. They are also expected to develop intervention programs or rework policies to eliminate these discrepancies. The CDO works alongside the unit administrative leaders to help them achieve both. Units report their efforts to the Provost and Committee on Diversity and Climate. When asked if these efforts were assisting the campus in its overall goals to be more diverse and inclusive, the Provost answered, yes. According to her, individual units were taking more ownership and responsibility for themselves and learning in the 
process. The practice of producing annual reports and presenting their collective efforts to the larger campus community created accountability. The Provost commented, that the groups get to "watch each other, we get to talk about it...they are finding what their problems are" and are addressing them with the support of the group. She added, "that makes it much more sustainable because they are learning from what they present and conclude to us" and are more motived to make improvements.

At Francis University, the CDO shared that outcome measures are a part of every project and initiative she takes on or asks others to participate in. With strategic partners such as the Vice Provost for Faculty, or the Vice Provost for Enrollment Management, the CDO identifies the desired outcomes of any initiative, the ways in which the outcomes will be measured, and how they will be assessed. The outcome measures identified by the CDO and her colleagues are shared with the wider campus community so that transparency and accountability occur. As the CDO put it "that's the way we do business." A research associate in the CDO's office assists her in compiling reports on specific areas they are monitoring, such as faculty retention, and student achievement among historically underrepresented minority groups.

Central University based most of its outcome measures on compositional diversity. Most of the efforts they identified were directed at increasing the numbers of historically underrepresented students, faculty, and to a lesser extent, staff and in strengthening the climate for these groups. This was due in large part to the low racial diversity in the state, which is predominately White by a significant proportion. Given this fact, attracting and retaining diverse individuals students to Central University becomes very critical. To account for serving underrepresented students, Central 
University has an organized structure in place in each of the colleges and schools to serve "minority and disadvantaged" students. A coordinator and a committee of faculty and staff in each school track the school or college's efforts in serving and retaining lowincome, first-generation, and underrepresented students. All of the coordinators report to an individual in the CDO's office who works with the coordinators in analyzing the minority and disadvantaged student outcome measures, which include academic achievement, time to degree, and graduation rates. This structure connects the CDO's office to the academic units in a coordinated and mission driven manner.

As shared in the beginning of this section, the outcome measures used by the campuses varied, as they were customized based on the needs of the individual units, schools, colleges, and divisions within each campus or on a particular initiative. However, outcome measures did play an important role in the CDOs work. In particular the CDOs were responsible for the frequency and operation of many of the campuses' assessments measuring diversity and inclusion efforts. The CDOs were also responsible for using the data from institutional data sources and the campus climate assessments to help the units within their campuses identify outcome measures appropriate for their desired diversity goals.

\section{Key Strategies to Institutionalize Diversity and Inclusion}

In response to research question three; what strategies contribute to successful institutional efforts to sustain diversity and inclusion at the three exemplar universities, the data indicated that the CDOs and others utilize various strategies and tactics to support their efforts. However, five key strategies emerged from the data at all three case study sites. The CDO is evident in all five and is integral to each strategy. A detailed 
discussion of each strategy and the evidence supporting it follows; however, for purposes of introduction, the five strategies are noted here. First directed by the CDO, each campus used a campus-wide diversity plan to guide institutional efforts and to address accountability. At some campuses individual units created their own diversity plan off of the campus-wide plan. Second, through the leadership of the CDO, each campus analyzed the data carefully and took deliberate steps to understand what the data was really revealing about populations within the campus community. Third, related to the data in strategy two, each campus in conjunction with the CDO took deliberate steps to share the data widely amongst the campus community. Sharing of the data resulted in professional development trainings to discuss the findings and to identify related solutions. Fourth, the CDOs and the Provosts used incentives to reward and sustain acquired knowledge competencies and efforts developed through the course of the institutionalization effort. Fifth, each of the campuses assessed the external environment to understand how external forces could assist or subvert ongoing diversity and inclusion efforts led by the CDO. These findings and related strategies are important because each one and the evidence supporting them provides guidance and context for campuses to consider as they engage in attempts to institutionalize diversity and inclusion at their institutions. These findings also go deeper than the existing literature on CDOs and provide specific examples of how the CDOs and their respective campuses in the study are attempting to transform their institutions toward diversity and inclusion.

\section{Using Campus-wide Diversity Plans}

As indicated earlier in this chapter, each of the campuses in this study all had diversity plans that the CDOs used to lead the campus in its diversity and inclusion 
efforts. The CDOs in the study credited their campuses' diversity plans as instrumental for organizing their campuses' diversity and inclusion efforts and for holding campus constituents accountable. The Provost at Morrill University attributed her campus' most recent successes to the fact that the campus had a diversity plan that for the first time held units accountable. She had this to say:

One of the things that we have, which I don't think a lot of our peer institutions have, is we clearly have a plan - it's not a perfect plan, but we clearly have a diversity plan with a lot more accountability tied to it than we've ever had before. So has [Morrill University] always paid attention to diversity or cared about diversity? Yes, I think we have. But one of the things that we've always heard from faculty, staff and students on campus, as well as what we know from the research, is that accountability plays a big piece in it....Are we closer to that? I think we're getting there. I think that the last four and a half years, with the diversity plan and what we're doing, and rewarding units for progress - I think that's a step in the right direction.

A colleague at Morrill University also noted that accountability was the significant change from previous diversity plans. He shared:

We've always had a diversity plan, but that's all it was, it was a plan. There was no accountability for the plan. This accountability report now requires us, every two years, to look at what we're doing, to report out what we're doing at a very high level, to the provost and the president, and now the vice-presidents and the deans.

To this point, Morrill's Provost shared a similar sentiment about previous diversity plans.

She shared, "we had [the plan] for three years; nobody even knew we had a plan. I shouldn't say 'nobody,' but lots of people did not know about it. It belonged to the [CDO's] office. It did not belong to them." To address this problem, she and the CDO asked administrative and faculty colleagues and students for their feedback. The responses she received explained that the plan was not developed collaboratively and therefore, there was no true ownership for it. It also had no accountability to hold people responsible or reward those who were serious about the work. With that information in 
hand, the $\mathrm{CDO}$ began to gather feedback from all of the campus stakeholders and listened intently for ways to gain broader support. The CDO re-wrote the diversity plan with the feedback she received. As she was rewriting it, the CDO shared updated versions of the plan to campus partners along the way leading to the version of the plan Morrill University is currently using.

To support Morrill University's new accountability measures, departments and units were encouraged to create their own diversity plans to guide their efforts in the campus' overall reporting process. For example, Student Affairs was working on a divisional diversity plan exclusively for Student Affairs. They were using the constructs of the campus' diversity plan to layout strategies and metrics to assess improvement in their specific departments. From this they had developed their own internal committee to pull data that they wanted to report and identified specific programs to highlight to the evaluating committee. The Assistant Vice President of Student Affairs shared:

We've embarked on trying to create a strategic plan for diversity within the division... at the Vice President's level....We've had some very good success in really looking at who we are as a division, how we're using the data. So we have a process in place... When we have to do [the campus diversity] report, we go around and we really ask our departments, 'What efforts have you engaged to retain diversity in your area? What efforts have you gone through to recruit diversity? What are your plans or efforts to improve diversity and, if you have an advisory group, what's their purpose?' So we're basically mirroring the things that are asked on that accountability report, and to have each one of those departments illustrate what they're doing.

Periodic updating was also a theme at Central University, where they have operated under some type of a diversity plan since 1988. At the time of the study, Central was developing its fourth campus diversity plan. According to the Provost at Central, it was time to redo the current diversity plan because a lot of change had occurred in the last eight years. As he described it, "life has changed dramatically." As 
an example, he shared that for the first time the state and university are experiencing an increase in Southeast Asian immigrants migrating to the state's rural areas. The campus had not considered climate or student and staff experiences for this population. In addition, there had been changes in leadership and a reorganization of administrative divisions. During this period the campus hired it's first fulltime administrative CDO. Previous CDOs had been halftime appointments of a faculty member. For purposes of the most recent diversity plan, the Provost requested to the CDO that the ad hoc committee writing the report divide the report into two sections to address near term and long-term efforts. He described his vision in this manner:

I suggested we needed an action plan that has two components: one that has a half-life in the zone of three to five years, something that we would pay attention to what's going on right now on our radar scope; and then a broader, more gradual plan that sets the future agenda for the next five years. So it's an overall ten-year plan, with the front side having a sharp focus, and then the backside more gradual.... Nothing is immutable, you know, that's the whole point. The front side we work actively on; we keep in mind the targets of the backside.

As the ad hoc committee was writing the plan, it engaged in yearlong listening and feedback sessions across campus with all of the campus stakeholder groups. According to the Provost and the CDO, accountability had to be a key feature of the new plan. As a result, the Provost was charging a separate committee to work on the implementation of the plan to delineate accountability. The $\mathrm{CDO}$ would serve on this committee as an exofficio. According to the Provost, the next step for the diversity plan would be "to take the framework of the plan and translate it into action items" for the various colleges, schools, and administrative units at Central University. The ultimate goal will be for units to develop their own diversity plans similar to what is taking place at Morrill University. 
Francis University has also operated under a diversity plan guided by the CDO.

At the time of the study the $\mathrm{CDO}$ was getting ready to lead the campus in its next diversity plan. Most recently, however, under the leadership of the president and provost, the campus completed a strategic plan for the entire institution where diversity and inclusion were interwoven into the plan. Two of the participants interviewed at Francis commented that their participation in the campus' strategic plan utilized context from the diversity plan. One had this to share,

We recently also did a campus-wide exercise...to draft...the strategic plan. Academic units were asked to do that; colleges, as well as the university in general, and a very big part of that was a discussion about values and goals, and diversity and inclusiveness, in terms of our long-term projections. So from the president, to the cabinet, as well as all of the academic units, we've been asked to think more deeply about what we're doing and where we're going, and how this connects to the university's identity and mission.

Another participant at Francis University shared that the diversity plan and campus strategic plan helped her department frame it's purpose in the larger institutional context. According to her, this process aided her staff in understanding where they fit in the campus' goals toward diversity and inclusion and the mission of educating the whole person. These examples highlight how the diversity plan with the CDOs' leadership was helping members of the campus community think strategically about diversity and inclusion.

\section{Analyzing the Data}

In each of the three case study institutions, data is a key strategy in sustaining the efforts of the campuses' diversity and inclusion work. As one participant stated, "data is everything." Data is important because it informs the campuses where they are at and as the $\mathrm{CDO}$ at Francis University described, "it moves [the campus] along to where it needs 
to be." In addition to informing campuses of its current realities, data aided institutional leaders in making informed decisions about being more diverse and inclusive. Data also assisted the CDOs and others in broadening the understanding of diversity and inclusion on their campuses with sound evidence.

At Francis University the CDO admitted that before her arrival the campus was collecting data but was not utilizing it in any constructive manner to inform the change process toward greater diversity and inclusion. She shared the following comment:

Yes, they were collecting a lot of data because of federal government regulations. But they were not doing anything with it; nobody knew anything about it. It was collected; they compiled it and held on to it.

For her, it was important to start using the data to inform the campus of where it was and to challenge them to improve. According to her, "you just have to know what data to use to drive them." To illustrate where the campus was and where it could be for historically underrepresented minority students, she utilized the Equity Score Card tool developed by higher education researcher Estela Bensimon (2010). For her, use of the data was important in supporting accountability. As she described it, "having that data and that system of accountability for units to see, 'this is where we were; this is where we're going' or 'this is where we slipped back." In order for all the units at her university to take responsibility and ownership they needed to know that "all of it is important." To further drive home the point of progress and meeting targets, her office publishes an annual report highlighting the improvement or worsening of each department and mails the report to all of the department chairs. As she shared, "nobody likes to get a zero" on their report. A member of the faculty agreeable to this practice explained how, "the scorecard gives an objective data presentation on how the campus is growing in terms of building 
diversity across academic units and across the campus." Without this transparency, faculty and departments might be more reluctant to act.

At Central University, data assisted the $\mathrm{CDO}$ in educating his colleagues. For him showing data in combination with several other strategies helps to transform the campus and make it more diverse and inclusive in its behaviors and attitudes. He shared:

If you can point to substantial data, because, 'We're a Research I institution. Show me the numbers. I want to see the data.' If the data can support the claim, then that also changes the conversation. And I think, with that sort of multipronged approach: getting data; building the appropriate relationships; having the right balance between administration demonstrating that this is a campus priority and is not just lip service, but that we're committed to this and that we expect our deans and, accordingly, our chairs and, accordingly, our staff to respond ... now you're having a different conversation.

A faculty member at Francis University had a similar perspective to share regarding data.

He explained it in this manner:

So I think when you're talking with people, and you're not just talking about what you think or what you feel, but you can actually point to hard data that shows that institutions that are making headway or showing outcomes and gains that are positive -- that data, I think, and that evidence, is far more persuasive than just my trying to get somebody to come on to my side of the argument... In terms of my colleagues, I would say that generally, people are much more persuaded by sound theory, good data, and good evidence.

For this reason, the $\mathrm{CDO}$ at Francis reported that every argument she makes about how the university could do better to enhance the learning and development of students or the retention of faculty; all of it is cited. She claimed, "everything I do is cited. It's always research. The research comes first." If she can point to the latest research, she felt she could make a case for change.

Data from peer universities and evidence-based practices helped demonstrate the direction in which universities could improve. At Morrill University the Provost allowed the colleges, schools, and administrative units to select their own peer institution or 
national organization for benchmark comparisons. For her it was important that the units select their own benchmarks in order to create buy-in because previous arguments using general comparison were sometimes misleading and unfair. The Provost explained the problem:

For example, we always counted faculty and students; but what we heard over and over again, 'You're using the wrong yardstick. There's not that many women in Aerospace Engineering - there's a whole lot of them in Education. Why are you treating us like we're the same no matter what our percentage is?' So, for us to hear them and say, 'Okay', but if we benchmark against other units - whether you're Student Affairs or the Division of Finance or Geosciences - if we tell you, 'you pick who we benchmark against' and we benchmark against that, and you're supposed to be a national leader in this area - so prove to us you're a national leader in this. It reduced the arguments against it, because I'm really not trying to see if we can be as gender-diverse in Education as we are in Electrical Engineering. But I am wondering why we're not as African-American diverse in Electrical Engineering as they are because the data would tell me we can be.

She went on to say that since the change in identifying appropriate benchmarks they are seeing greater acceptance toward the process and overall improvement. According to her, "now at the Deans' Councils, there's different kinds of conversations about diversity and what we're doing because their depth of knowing their own data and benchmarks, is better....They are thinking more critically." A participant at Francis University agreed that evidence-based practices from peer institutions are equally important in gaining support and sustaining efforts. He shared:

If we can...use evidence and evidence-based practices and point to what the research is showing, and if we can look at comparable universities, in terms of institutions that are similar in size, that are trying new things, either in student life or in academic life, those things generally tend to speak volumes in terms of helping to move the conversation along.

As the campuses in the study became more familiar with using data to inform their decisions, they developed new competencies that resulted in "digging deeper into 
the data" to get at trends beneath the larger data sets. The Provost at Morrill University explained how their process changed,

We got better with our own data, we got better at looking at the next level: okay 'you got them in - did you keep them?' And I'm not just talking about students; I'm talking about faculty or staff or other issues.

She and the CDO challenged the faculty and administration to find new ways to collect data to address these types of questions. She commented, "at first they would respond, 'Well we don't have that information"” and she would respond, "'but we could [have that information]. How shall we get it?"' The CDO echoed the importance of collecting data and encouraged staff to conduct more assessments. She shared:

We ask individual units - the colleges or the non-academic units - to do some more assessment at their level, which the data doesn't always get you to at the large university level. To drill down and see: what are people experiencing in their own units? Are we seeing any patterns at the unit level versus the campus level?

The theme of drilling deeper into the large institutional data sets continued, as participants, wanted to know more about "what was the data saying or not saying." At Central University the CDO's office was disaggregating data collected by the institutional research office that reported data in broad categories. When not carefully examined, these data sets could report a false picture. A diversity administrator in the CDO's office shared:

When we look at faculty and staff we don't differentiate between domestic and international. And so when we look at, say, faculty of color it looks like there's a large number, but a lot of them are international faculty. So, especially, AsianAmericans are not pleased with that, because they say a lot of the 'Asian' faculty are really international faculty from India, China, Japan.

This reporting can also skew climate data for Asian-American faculty who may have a different experience working in the university. The same argument for drilling deeper in 
the data applied to disaggregating data among gender and people of color. According to the same diversity administrator,

I push very strongly for dis-aggregating women of color faculty, because when you're looking at gender and then race and ethnicity - just individually - it looks like the number of women faculty has gone up. It's now $22-24 \%$; the percent of minority faculty has also gone up. But when you look more closely, and you cross gender and race/ethnicity, the number of women of color is a big issue. So it's got to be looked at more closely because we should be addressing that particular issue, not just race and gender.

A Student Affairs administrator at Francis University shared a similar sentiment.

At the encouragement of the $\mathrm{CDO}$, it was critical that she and her colleagues disaggregate the data for first-year retention rates by gender. They were noticing different trends within the same ethnicity when divided by gender. She explained that for her unit, "there are pieces that we need to start digging at a little bit deeper into the different ethnic backgrounds, because it will give us a better look." Central University and Francis University offered examples of how their efforts required a deep dive into the data, which then allowed for more strategic approaches that could improve the campus' diversity and inclusion efforts. The CDO as the lead analyzer of this more precise set of data could then advocate for change and help individuals at each site dive deeper into the data to get better results.

\section{Educating Campus Stakeholders Using Data}

In conjunction with the deep dives in the data and transparency, each of the campuses in the study engaged in intentional activities to broadly share the data so that faculty and staff could not only become aware of the climate and conditions of the institutions, but could act accordingly to contribute to the change process. This actionoriented direction was facilitated through trainings and professional development 
activities assisted by the $\mathrm{CDO}$ and their offices at each of the campuses. At Morrill University after data from the university-wide campus climate survey was analyzed, a group known as the Diversity Operations Council directed by the CDO convened a workshop session for faculty, staff, and students to review the findings. Over 130 participants attended. The purpose of the workshop was to review the data with the various campus stakeholder groups but more importantly was to "dialogue [on] what the data means," so that the campus could work together to address the needs expressed. As the CDO described:

We had people engaged from 9:30 this morning to 1:00 this afternoon. We had break-out sessions....It's a work session, so we presented the data and then said, 'Okay, we're going to break you up into small groups of a faculty, staff and a students, and help us to generate some ideas in terms of how to best move forward, based on our challenges and some of the successes that we're having.' In these sessions the purpose is not to hide the data but be transparent so faculty and staff can learn and make appropriate adjustments.

At Francis University a similar approach was taken to share the data from the most recent climate survey. After the results were analyzed, the data was shared with the CDO who convened a group of faculty and administrative leaders to address the findings. A participant of this group from Student Affairs reported after this session, "a strategic tactical planning meeting occurs" where they "go over the most important pieces that need to be addressed." She credited the leadership and guidance of the CDO in helping the campus approach the results as a "team." As she described it, "we're looking into this as a team, 'how do we do this, so it doesn't feel like one department is handling all things,' but rather the entire division, the entire campus is part of [the solution]." In this example the collaborative work described is important because it empowers more individuals to engage in the work of transformative change. 
It is also important to note that while being transparent with the data and hosting open sessions to educate the campus community on the data for purposes of finding solutions, some participants in the study expressed frustration that not enough is being done. For example, one participant expressed:

There's still a high level of dissatisfaction among our women faculty and our students of color here, primarily our African-American students. And that's nothing new. You know, we're looking at data that we've collected over fifteen years and those were the two primary issues fifteen years ago and they're still the issues that we're dealing with today.

For him, he stressed that more education and accountability had to occur. Overall he was pleased with the direction his campus was going and the leadership of the $\mathrm{CDO}$, but he stated more could and needs to be done "to educate the rest of the campus." He shared that this observation was also troubling the staff he managed because they were developing "a lack of trust," as he described it. A professional diversity consultant was brought in by his division to help the staff be more engaged in diversity and inclusion work directed at students. The consultant shared with the Assistant Vice President that the staff was unsure about the "institutional commitment" of the campus to alleviate ongoing problems experienced by marginalized populations. He shared that the consultant,

kind of alluded to us that there are still some issues of trust...around this topic. [The staff's] question was, 'Okay, we're having these great conversations, but what's next?' And [the consultant's] challenge to us was, 'continue the dialog, continue the conversation.' I think our employees think that we're just going through this as part of our exercise, and that there's no real true commitment from the leadership.

He and his managers were committed to address this point with their staff. A strategy he identified was regular professional development trainings on diversity and inclusion topics that the staff identified as important for student wellbeing and for their own knowledge competencies. These diversity and inclusion themed trainings occur every 
one to two months. Previous trainings have included topics such as student veterans, students with disabilities, and gender differences. He shared that these are some of the most popular and well-attended professional development offerings, which according to him is important because they also enforce broader concepts of diversity. While he shared that some staff push back on the frequency, he feels there is,

enough turnover in the division, and we have enough entry-level employees where this is new to them, this is professional development for them, so they want this....But with the majority of our people, when you look at the turnout of the programs, they want to learn how they can sharpen their saw or add to their toolbox. So they see this as value added.

Various professional development strategies continued as a theme throughout the interviews. At Francis University the President invites faculty and staff to participate in a leadership institute that discusses the campus' mission and future. Discussions on the principles of diversity and inclusion at the campus are a significant part of the institute and are lead by the CDO. In another strategy the CDO invites faculty who are the recipients of grants awarded from her office to report on their projects and results to fellow faculty. The grants she awards are for faculty to address some element of diversity and inclusion in the classroom. In this situation, faculty are sharing with fellow faculty promising practices, which for some is a more convincing approach and provides them with the support to believe that diversity and inclusion work is possible inside the classroom. A member of the faculty indicated that these practices were an effective strategy. According to him, "these are ways of celebrating faculty who have actually begun to buy in and begin to show some transformative experiences where they're incorporating this into their research, their scholarship, and their teaching." 
At Morrill University the CDO's office offers faculty and staff trainings on difficult dialogues and mediation, and provides a seminar to faculty on conflict management. Her office also supports a speaker series that bring various accomplished speakers to campus to discuss diversity related topics. According to the $\mathrm{CDO}$, she feels, "it 's important for the university community to see individuals across the country who engage in diversity at the scholarly level." At Central University the campus hosts faculty trainings for diversity hiring. They also host a forum once and sometimes twice a year, where institutional data on diversity and climate are discussed. The Provost shared that "it is an active program" with broad participation. The CDO at Central shared that in his previous capacity as a faculty member, he provided professional development training for faculty and staff using theater techniques. By using theater he was able to broach difficult topics about race, sexuality, and gender that were often avoided in other types of traditional staff trainings. He also credited these as fairly successful and well attended by staff. As one participant described, professional development and staff training is about "providing staff and faculty with the sense of empowerment to do the work that they need to do."

\section{Providing Incentives}

At each of the three campuses incentives were used to move the campus along in its diversity and inclusion goals. Some of the incentives related to diversity and inclusion efforts were awarded by the Provost and some by the CDO's office directly. However, all of the incentives identified in the study were used to reward and sustain knowledge, competencies, and new efforts developed through the course of the institutionalization. At Morrill University the Provost and the CDO use incentives to get deeper change out of 
the units during their biennial reviews. The Provost sets aside one million dollars to be given as awards to units who demonstrate marked progress in the three goal areas: accountability, improved climate, and equity. She explained, that the monetary incentives were "incredibly important to launch the process" to trigger institutional change. She admitted that it was a learning process, but according to her, "they eventually figured out, that you have to be in all three areas: benchmark, climate and equity - doing some remarkably innovative stuff or be clearly in the front of the benchmark national pack - to qualify for the money." She also stated that, "you can be doing a good job, incrementally better than you did last year, and you won't qualify for the money" because they are all expected to be steadily improving. For her, the rewards are given to those who provide more than quick fixes to address a particular problem. Recipients have to be working with the data and the CDO intentionally to make changes that will get at underlying assumptions rooted deep in the institution. As she described the process, its not "just giving them prizes for doing intervention programs. I believe in intervention programs, but I'm like, 'No, no. There's got to be deeper change than that."' She admits that, "now, it's kind of a competition, I believe a healthy competition. And now they get a lot more engaged when they present." She provided one example:

The first year, about forty-percent of the deans came and presented their own benchmarking data. Otherwise, they let their associate dean for diversity do it. By the third year, most of the deans had figured out that the council that reviews this - which has some students and some faculty, and some administrators and some off-campus people - were more impressed when the big dog comes and does it. So now they come and present it, and can dialog.

The Provost did stress that the money could be used in any manner the receiving units choose. Direction on how to spend the award or where to spend it was left to the units to decide. The Provost and the CDO were wise enough to predict that units who 
had not received an award might use this as an argument as to why they could not meet

the benchmark data. To this point the Provost shared:

That argument that 'I can't improve my climate so that more diverse people can thrive here' means that your underlying assumption - says that you're not really for diversity. Because if I said, 'You need to improve research,' you would find the money in your budgets to improve the research environment for your faculty. So we had a lot of conversations about: if our underlying assumption is 'this is not at our core ... this is on top of ... on the side of ... an extra icing that we can give, but not the real cake' - then that says what we think diversity is. If this is as much a part of what will make us a university - as being better researchers and better teachers, then you have it in your budget.

For her the rewards were simply for those who were excelling and making deep

transformational change in their units. Student Affairs at Morrill University had received two awards totaling $\$ 70,000$ in the two instances they have had to report their efforts.

Despite a range of areas where the money could be allocated, Student Affairs decided to use the money in their diversity and inclusion work in the department. As the Assistant

Vice President described it:

What we decided to do here, with the blessing of our Vice President, is put all those funds back into our diversity initiatives. So what we created is our Campus Climate and Inclusion Funding. So, basically, our departments can apply for funds and primarily it has to be for training or professional development. And it's not for students; it's for staff only. So we want to see you use these funds to better your department.... This is strictly to help your department better understand and appreciate diversity.

At Central University the CDO considers incentives to support new faculty

research that supports diversity. He explained,

If there is a new research enterprise that a faculty member wants to develop, if it has direct correlations with diversity in the broader sense, if we can draw a dotted line, if you will, to what happens in that academic effort to what happens in this office, certainly we'd be happy to explore putting resources towards it.

Additionally at Central, as indicated earlier in this chapter, the Provost at Central allocates a million dollars to help with the recruitment and hiring of diverse faculty. The 
award pays the hiring department three year's of the new faculty member's salary, allowing the department to hold onto this cost saving for other needs. The Provost shared, that sometimes faculty "complain that there's not enough, but there are plenty of resources. I tend to be a performance-driven person. I mean, I'll give you the resources; you show me the productivity."

At Francis University the CDO has used incentives to reward departments and administrative units with specific projects that strengthen the campus' diversity and inclusion efforts. Many of these incentives are funded through grants the CDO is a recipient from external agencies seeking to support diversity and equity in higher education. One example of a grant she received provided $\$ 5,000$ awards to faculty and staff for new diversity and inclusion initiatives. Two of the most recent awards given by the CDO were to assist a gender equity project and a program for former foster youth at the university. The $\mathrm{CDO}$ at Francis had another pot of money to incentivize faculty to restructure their upper-division courses to incorporate elements of socio-cultural contexts that bring in aspects of diversity, equity, and inclusion into the course material, regardless of it's subject matter. For her this was an initiative of particular pride because it was about transforming the classroom experience to support diversity beyond the mandatory lower-division "diversity" courses, which to her were merely a "requirement." According to her, money to restructure upper division courses was more effective at transforming the curriculum and educational experience of Francis University students. As she shared:

Quite a few faculty over the years have gotten a grant to restructure their curriculum of upper-division major courses that are not diversity-oriented courses, but rather courses that everybody has to take if they're going to get a major in, say, Geography. And so that means that students walk out of here with more of 
the notion that diversity is not something 'over here' and then you go and do your work 'over there'. It's part of the way of doing business at Francis University.

After the external grant ends, the CDO takes the outcome measures and data collected during the period the initiative ran, and makes a case to the President to institutionalize the program with permanent funding. This has been a successful way for the CDO to test out new initiatives with non-university resources to see if they are effective practices for the university to permanently adopt. For her grant writing is a just part of her job. As she described it,

I would say that [applying for grants] it's just part of the job that I do, because you have to ... Grant writing and getting grants is a timely thing; you have to know when what agencies, and what foundations happen to have a call out for a grant. They're not out there every day; it's only when they're out there you have to act.

Grants she has participated in have come from private organizations and the federal government. She is continuously looking for new opportunities to partner with organizations or benefit from agencies looking to improve the culture of higher education to be more diversity-minded. These partnerships add to her financial resources to provide incentives for the campus to change.

\section{Assessing the External Environment}

Finally, each of the campuses assessed the external environment to understand how external forces could assist or subvert ongoing diversity and inclusion efforts led by the CDO. The Provosts in this study expressed the most concern as they were trying to preserve the work of the $\mathrm{CDO}$ and the progress made by the campus. Awareness to the external environment is an important strategy because it is the only strategy of the five that intentionally looks outside of the university to understand what may impact the university internally. For example, the Provost at Central University was concerned with 
over "dependency" on their K-12 pipeline programs at the university. He expressed concern that over reliance on these programs for the institution's undergraduate diversity could be problematic should anything disrupt the system in which they function, such as enrollment management not meeting their admission targets. He also was concerned with the perpetual need to raise scholarship money to support these programs. According to him, the university had done a great job in this area thus far but as he commented, as a public university they could not discount their tuition as private universities do to attract underrepresented students. Furthermore, the Provost was keenly aware about restrictions imposed by the state legislature to cap non-resident student enrollment to twenty-five percent at its public universities. Because the state was so heavily White, this was a challenge to the Provost who felt his opportunity to get more diversity into Central University would be aided if he could recruit more students from out-of-state and internationally. Thus he and the CDO were looking at new opportunities to have this policy changed.

At Francis University the Provost was cognizant of pushes to bring more international students to the campus as well. However, as he explained per agreements with the city where his university is located, it is at capacity in terms of enrollment and facilities expansion. To increase the student enrollment in one area would mean to decrease it another. He stressed that while these conversations were occurring it was important to ask how they might disrupt current diversity efforts for domestic students. As he put it, "it might and it might not," but according to him the campus "has to be particularly vigilant not to let that happen" as they engage in future planning. As he described it, "if you're not careful, you can get into sort of conflicting disagreements." 
For this reason the $\mathrm{CDO}$ is present at these sorts of discussions on institutional forecasting.

As a public institution, Morrill University was under similar pressures from the state. The state legislature was carefully watching the university to make sure it did not exceed non-resident or international student enrollments. The state has articulated a clear directive that the focus of its public universities is to be educating the citizens of the state. However, unlike Central University, Morrill University is located in a state that has a history with legal rulings involving race based admissions practices. In 2004 the president of the university made a proactive choice to end the institution's practice of using race or ethnicity in its admissions practices. As a result, the campus refocused its strategies of recruiting underrepresented minority students by focusing on geographic regions within the state with high concentrations of diversity and potential firstgeneration college students. Several recruitment outposts of the university were established in various cities throughout the state. This strategy has proven successful but is not without it's challenges. A significant amount of institutional resources are directed at maintaining these centers. Additionally, unlike the pipeline programs established at Central University, these recruitment centers are not guaranteed admission strategies. As the Assistant Vice President for Admissions shared, "at the end of the day, I'm charged with getting the most academically-prepared diverse class possible without using race as a factor." The pressure to deliver this class is intensified by state legislators, institutional leaders, alumni, donors, and faculty who want to know how many African-American and Latino students his operation had admitted.

\section{Summary}


In conclusion, this study yielded rich data related to all three research questions. Major findings from this multiple case study examining three universities who have institutionalized diversity and inclusion and have a CDO, revealed the following major themes. First, the role of the CDOs is to lead the various change initiatives and efforts to institutionalize diversity and inclusion on their respective campuses. To do this, CDOs work at the highest levels to lead systematic and integrated change efforts and serve as a centralizing resource and point person. As change leaders, CDOs operate with intentional strategies that require relationship building to work with campus partners. As such, CDOs are aware that institutionalizing diversity and inclusion takes time. They are also aware that there is perpetuity to the work and that it is never really done.

Second, data from all three campuses in the study indicated that the institutionalization of diversity is evident when attitudes and behaviors surrounding diversity and inclusion shift from traditional notions of diversity to broader understandings of the complexity of diversity and its benefits to the university. A broader understanding of diversity includes awareness that diversity is more than counting numbers and exceeds traditional notions of race. It includes gender, sexual orientation, economic levels, and other forms of diversity including but not limited, to student veterans, students with disabilities, former foster youth, and students from mixed-race backgrounds to name a few. As part of this broader understanding the universities in the study gave additional attention to campus climate because it relates to how welcoming the campus is to diverse people. Thus, with their more complex understanding of diversity, the campuses recognized that diversity efforts also had to address inclusion. Outcome measures the campuses used to determine their institutionalization efforts 
varied between the case study sites and were not uniform throughout each campus. However, the CDOs in each case study site worked with the units within their institutions to come up with appropriate outcome measures based on institutional data that the CDOs analyzed and used for assessment purposes. As the lead person analyzing institutional data on diversity and inclusion, the CDOs in the study worked on accountability systems to hold units within their respective campuses responsible.

Finally, data from the study indicated that the case study sites utilize various strategies and tactics to support their campus' diversity and inclusion efforts. However, five key strategies emerged from the data at all three case study sites: using a campus diversity plan, analyzing the data, educating stakeholders using the data, providing incentives, and assessing the external environment. As was discussed, the CDO is evident in all five and is integral to each strategy. Together, the findings and strategies from this study go deeper than the existing literature on CDOs and provide specific examples of how the CDOs and their respective campuses in the study are attempting to transform their institutions toward diversity and inclusion. A discussion on these findings, how they relate to the literature, future research, and proposed recommendations for practice follows in Chapter 5 . 


\section{CHAPTER FIVE}

\section{DISCUSSION}

\section{Overview of the Chapter}

This chapter begins with a brief overview of the purpose of the study, the research questions, and a summary of the findings. It is followed by a discussion on the interpretation of the findings and linkages between the literature and the results of the study. It concludes with implications for institutional practice, areas for future research, and a final summary on the study.

\section{Overview of the Purpose of the Study}

The purpose of this study was to examine the role CDOs play in leading efforts to institutionalize diversity and inclusion that results in transformational change. A further purpose was to understand how the three campuses in the study, with the support of their CDOs, have institutionalized diversity campus-wide so that diversity is a sustained institutional effort with measurable outcomes. In this study, institutionalization is defined as the establishment of a standard practice or custom in an organizational system (Curry, 1992; Kramer, 2000; Kezar, 2007) and is considered the final phase of a transformational change process toward a particular goal (Curry, 1992). In this study the goal is diversity and inclusion. To institutionalize diversity and inclusion means "leaders are engaging in the process of moving a diversity agenda forward to institutionalize a new way of doing work" that is stabilized and permanent (Kezar, 2007, p. 415). A successful institutionalization effort will elevate the role of diversity and inclusion and protect it even in times of economic difficulty or leadership uncertainty (Williams, 2013). 
The role of CDOs and the institutionalization of diversity in higher education are important aspects to study in tandem because they shed light on the actual versus perceived operationalization of diversity efforts in higher education. To institutionalize a practice like diversity and inclusion requires organizations to modify reward structures, policies, and the environment (Kezar, 2007). A CDO who is committed to leading a change effort that empowers others, holds people and administrative units accountable, and maintains a focused and integrated approach to the institutionalization of diversity and inclusion, helps institutions reach the final phase of a transformational change process. With a more in depth understanding of the transformational change process via institutionalization, this study aims to contribute new data to the field by examining exemplar colleges and universities that have a $\mathrm{CDO}$ and have excelled in efforts to institutionalize diversity and inclusion campus-wide. Additionally, this research adds to the literature and contributes promising practices to university leaders considering hiring a $\mathrm{CDO}$ and or looking to institutionalize diversity and inclusion at their respective institutions. Three research questions guided the development and design of this study. They were:

RQ\#1. What role do CDOs play in institutionalizing diversity and inclusion in US universities?

RQ\#2. How do three exemplar universities know they have institutionalized diversity and inclusion? What are their outcome measures and who is involved in these efforts? 
RQ\#3. What key strategies contribute to successful institutional efforts to sustain diversity and inclusion at three exemplar universities? What is the role of the CDO in these efforts?

\section{Summary of the Findings}

This study yielded rich findings related to the research questions. First, the role CDOs play in institutionalizing diversity and inclusion is to lead transformational change by institutionalizing diversity and inclusion into the broader campus community. The CDOs in the study did this by using a systematic, relational, and integrated approach. To lead their transformational change effort the CDOs in the study carried the message of diversity and inclusion to all corners of the university so that it could permeate the wider organization and result in changed attitudes and behaviors among campus stakeholders. To have this broad effect, each CDO in the study worked at the highest levels in their respective universities to effect change and to serve as a centralizing resource. As change leaders, the CDOs operated with intentional strategies that required relationship building to work collaboratively with campus partners. Although all the CDOs in the study had high levels of positional authority, they worked deliberately on their relationships to influence change rather than mandating it as senior leaders within a hierarchical schema. As change leaders, the CDOs in the study were also aware that institutionalizing diversity and inclusion takes time if it is to be deep and pervasive. The change process also requires ongoing efforts to sustain their work.

In addition to these findings, data from all three campuses in the study indicated that the institutionalization of diversity is evident when attitudes and behaviors surrounding diversity and inclusion shift from traditional notions of diversity to broader 
understandings of the complexity of diversity and its benefits to the university. This includes awareness that diversity is more than counting numbers and exceeds traditional notions of race. It includes gender, sexual orientation, economic levels, and other forms of diversity including but not limited, to student veterans, students with disabilities, former foster youth, and students from mixed-race backgrounds to name a few. As part of this broader understanding, the universities in the study gave additional attention to campus climate because the climate of a university indicates how welcoming the campus is to diverse people, particularly those groups that are historically underrepresented, marginalized or represented in smaller numbers at the institutions. Thus, with their more complex understanding of diversity, the campuses recognized that diversity efforts also had to address inclusion.

In support of these findings, the campuses in this study utilized outcome measures to determine the success of their institutionalization efforts. Outcome measures varied between the case study sites and within the institution. For example, the School of Business and the Division of Student Affairs within the same institution could have very different outcome measures due to the very different structure and cultures of the units. Nonetheless, the CDOs in each case study site worked with the units within their institutions to come up with appropriate outcome measures based on institutional data, which the CDOs then analyzed and used to assess their efforts. As the lead person analyzing institutional data on diversity and inclusion, the CDOs in the study worked on accountability systems to hold units within their respective campuses accountable.

The last set of findings to emerge from the data suggested five strategies that helped the CDOs and the case sites institutionalize diversity and inclusion. As the leader 
of the transformational change effort, the CDO played a lead role in all five strategies. First, directed by the CDO, each campus used a campus-wide diversity plan to guide institutional efforts and to address accountability. At some campuses individual units created their own diversity plan off of the campus-wide plan. Second, through the leadership of the CDO, each campus analyzed the data carefully and took deliberate steps to understand what the data was really revealing about the populations within the campus community. Third, related to the data in strategy two, each campus in conjunction with the $\mathrm{CDO}$ took deliberate steps to share the data widely amongst the campus community. Sharing of the data resulted in professional development trainings to discuss the findings and to identify related solutions. Fourth, the CDOs and the Provosts used incentives to reward and sustain acquired knowledge competencies and efforts developed through the course of the institutionalization effort. Fifth, each of the campuses assessed the external environment to understand how external forces could assist or subvert ongoing diversity and inclusion efforts led by the CDO. Identification of these strategies provides guidance and context for campuses to consider as they engage in attempts to institutionalize diversity and inclusion policies and practices at their institutions. These overall findings go deeper than the existing literature on CDOs and provide specific examples of how the CDOs and their respective campuses in the study are attempting to transform their institutions.

\section{Discussion of the Findings}

This section examines some of the major findings that emerged from this study and their meaning in the larger context of CDOs and their work at leading transformational change supportive of diversity and inclusion. Leading this section is a 
discussion on the work CDOs engaged in to elevate the importance of diversity and inclusion on their campuses, which included a systematic, relational, and integrated approach. Next this section focuses on the importance of a broader understanding of diversity to support transformational change. This is followed by a discussion on the challenges related to institutionalizing diversity and inclusion and the perpetuity of the work that makes it an ongoing practice. The section concludes with a discussion on new strategies that expands upon the work of Damon A. Williams and Katrina Wade-

Golden's (2013) strategies for CDO leadership, which is the most extensive research on CDOs .

\section{Elevating Diversity and Inclusion}

Darryl Smith (2009) explains that several decades ago universities understood the imperative to build capacity for technology. She writes,

technology was understood to be central, not marginal, to teaching and research. But more critically, technology was seen as central to the viability of every educational institution - that is, how the institution communicated, built infrastructure, spent money, and went about hiring. Because technology has been continually changing, institutions almost without question have been continually adapting as new technologies are introduced. Technology is now part of everyday life and every corner of institutional life. On some campuses, a new position has been created for a chief information officer, whose task is to develop strategies for incorporating future technological developments, for allocating resources, and for coordinating campus efforts (p. viii).

Smith concludes her point by stating, "we are now at a time when we must understand that diversity, like technology is central to higher education" (p. viii). Smith refers to this urgency as the diversity imperative. As such, the diversity imperative goes beyond traditional measures such as student success and looks at the entire organization's efforts related to diversity. Explaining the more recent and more informed perspective, Smith argues, 
the issue today is whether and how [emphasis added] institutions are building capacity to function in society in a way that is appropriate to their mission. In the next generation of diversity work, student success will be necessary but not a sufficient indicator of institutional effectiveness (p. viii).

This statement captures in large part what the three case study sites were trying to accomplish through the work of their CDOs. Rather than focusing solely on traditional notions of diversity, such as student support, the CDOs in this study were attempting to change the cultures of the organizations to be more diversity and inclusive-minded in support of their academic mission. Findings from this study suggest that the threeexemplar institutions are working toward the next generation of diversity work by striving to build greater institutional capacity for diversity. The campuses are achieving this through their CDOs who much like the chief information technology officers Smith described are leading campus-wide efforts to institutionalize diversity and inclusion through systematic, relational and integrated approaches to make diversity and inclusion a permanent feature of the organization.

The motivation and basis for Smith's argument is to create transformational change that moves diversity and inclusion from the margins to the center of the university's thought processes and decision-making. The CDOs in this study were actively engaged in that effort. One of the ways in which the CDOs did this was by working individually with administrative units and academic departments. Rather than using a top down approach, the CDOs worked collaboratively alongside their campus partners. As discussed in Chapter 4, intentional relationship building was essential to their work. The CDOs met their campus partners where they were in the change process. Those further along in incorporating diversity and inclusion into their hiring practices, curriculum, and/or reward structures required less one-on-one time from the CDO. 
However the CDO did not abandon all help. Rather, the CDO continued to support these units by encouraging them to continue with their efforts and to share their successes with other campus partners as best practices. For units struggling or showing more resistance to the change effort, the CDOs worked closely with these departments by helping them in the most appropriate manner conducive to the situation. For instance, the CDOs used data to demonstrate areas needing improvement and provided incentives through their offices or the Provosts' offices to motivate slower departmental leaders. The CDOs also provided professional development opportunities, seminars, and lectures to help the campuses understand the importance of diversity and inclusion in strengthening the campuses' mission and academic focus.

In addition, the CDOs worked to move diversity and inclusion to the center of the university by building trust. As one CDO shared, he was not interested in "naming, shaming or blaming," but rather wanted to work with campus partners to challenge them in an environment that was considered a safe space for candid discussions. To achieve this he had to work intentionally and strategically to build trust overtime. The CDOs also had to build trust and creditability from campus partners advocating for inclusive and diverse institutions. They did this by being transparent with institutional data and campus climate assessments and by holding units accountable for their actions. If the CDOs were perceived to be hiding information or glossing over aspects of the data that were less than flattering to the institution they would loose creditability amongst campus stakeholders advocating change. Additionally, if they did not hold units responsible for their efforts or lack thereof, then they would also lose credibility. Therefore, trust and transparency became essential to the CDOs in moving the institutionalization effort along and building 
a wide base of active supporters. Overtime the CDOs were transforming their institutions by moving the diversity conversation from the margins to the center of the university.

\section{Broader Notion of Diversity}

As campuses work to institutionalize diversity and inclusion it is also vital that they broaden their understanding of diversity and its various forms as the case sites in this study did. This is important because a broader notion of diversity widens the discussion and has the potential to allow more groups to see themselves in the change effort. Several of the case study participants in this study described practices and efforts that extended beyond traditional notions of diversity such as race and gender, which resulted in a more complex understanding of diversity and inclusion. At Morrill University the Provost, CDO, and Student Affairs administrators acknowledged that they needed to consider issues of LGBT students and faculty as much as they monitored climate issues related to the educational experiences of African-American students. They each acknowledged that this was especially challenging given the conservative climate of the institution, but understood LGBT diversity and inclusion as important, if it truly was to be a welcoming institution for all people as it inspired to be. At Central University consideration of socio-economic diversity was equally important to the campus due to a large number of students coming from rural farming communities. Academic success programs and services at Central University were also geared to White students as well as historically underrepresented students who came from rural and under-resourced communities. And finally, at Francis University the campus paid attention to climate issues affecting faculty, staff and students. Climate surveys and assessments were given to each group to understand how they experienced the campus community. The CDO at 
Francis shared that the campus did not have a problem recruiting or attracting underrepresented faculty. Yet, to ensure that underrepresented faculty remained on campus and thrived, the $\mathrm{CDO}$ worked intentionally with departments to create a welcoming and supporting environment. She also conducted interviews with faculty leaving the university. It was important to her to understand why members of the faculty left and if it had anything to do with the climate of the university being non-supportive to the individual based on their differences.

By extending their understanding of diversity and inclusion, the campuses in this study also expanded their notions of how diversity and inclusion benefits the university. At Morrill University, the $\mathrm{CDO}$ and Provost were intent on re-acculturating the institution to not think of diversity accountability as compliance or an exercise of counting heads. They wanted their administrative leaders and faculty to understand the benefit of diversity and how it strengthens the caliber of the organization and the quality of the education. As a result they were intent on creating a campus environment that encouraged meaningful interactions between diverse people and opportunities to learn and work together. In a message to the campus community on her website the CDO wrote,

We cannot begin to achieve [academic] excellence if we do not value diversity in all its human dimensions-age, gender, gender identity or expression, race, ethnicity, sexual orientation, nationality, culture, physical and mental ability, socioeconomic status, religion, and the like....no institution can achieve excellence without diversity.

A similar sentiment was expressed by the CDO at Francis University who used the Inclusive Excellence Model (Williams, Berger \& McClendon; 2005) to explain to her campus community the importance of diversity as a strengthening force for the learning 
environment and research of the institution. Francis University believed diversity contributed to the development of the "whole person," which in turn produced a better citizen of the world capable of solving society's most vexing challenges.

\section{Challenges and Ongoing Practice}

As CDOs at other universities attempt to institutionalize diversity and inclusion, a challenge sure to arise is working within an organizational structure that is decentralized, as many US colleges and universities tend to be. As the literature suggests, higher education is a complex loosely coupled organizational system with several overlapping cultures (Birnbaum, 1988; March \& Cohen, 1974; Weick, 1978). The CDOs in this study had to work intentionally with administrative and academic units despite the decentralized functioning of their campuses. This required consistent and integrative approaches by the CDOs to build support. However, this is an added challenge given that colleges and universities are not static organizations. For example, every fall a new class of students enters the university from across the nation and globe with a set of experiences and ideas that may or may not align with the institution's values for diversity and inclusion. In addition, new faculty members arrive and throughout the year there is staff turnover; both influxes require ongoing outreach and training about the campuses' diversity efforts and goals. Given these challenges, the work of the CDO becomes perpetual and integral in sustaining the institutionalization effort, an observation this study supported. Although success at institutionalizing diversity and inclusion was achieved, it required ongoing management by the $\mathrm{CDO}$.

Despite these challenges, the majority of the participants interviewed for this study agreed that their campuses had institutionalized diversity such that diversity was no 
longer a "novel conversation," as described by the $\mathrm{CDO}$ at Central University. It should be noted that this statement does not suggest that the campuses had achieved total harmony or perfection in regards to their diversity and inclusion efforts. Rather, they had made substantial progress in moving the campus from a "novel conversation" about diversity to a more thoughtful and purposeful discussion about how diversity and inclusion advances the university into a leading institution for the modern world. To underscore this point, the CDOs and Provosts in this study both acknowledged that ongoing efforts to continue institutionalizing diversity were still needed (in some areas more than others). A Student Affairs administrator at Morrill University echoed this point by sharing that despite recent successes, "there is still work that needs to be done."

Both observations suggest that there is an additional element to add to frameworks describing institutionalization efforts. For instance, the process of institutionalizing an effort such as diversity and inclusion is most often described in three steps: mobilization, implementation, and institutionalization, whereby the condition has been established and is now part of the organizational system (Curry, 1992; Kezar, 2007). However, what this breakdown fails to recognize, and what became evident in this study, is the ongoing efforts of the CDO to maintain the institutionalized outcome. As the Provost at Francis University described, "one of the dangers is to think you've got it right and therefore you don't have to keep working at it." For him the CDO would always have a job to make sure the campus did not fall back on its diversity and inclusion efforts they had worked to establish during the 12 years of the CDO's leadership. Therefore, given the dynamic rather than static nature of higher education in general, the need for perpetual attention to sustain the institutionalization effort underscores the importance of 
the CDO. It also turns much of the CDO's work at leading transformational change into a practice, which requires constant maintaining in order to preserve institutionalization effort they worked so hard to establish. While some may argue that the CDO is an unnecessary position within higher education, for those who have achieved noted progress and success, as was the case for the three institutions in this study, the CDO quickly becomes instrumental in the campuses current and future efforts.

\section{New Strategies for Institutionalizing Diversity and Inclusion}

Damon A. Williams and Katrina Wade-Golden (2013) have conducted the largest study on CDOs. Their research has identified several practices associated with successful institutionalization efforts led by a CDO. Among these include, a diversity plan, systems of accountability, informed search processes, professional development and trainings, and incentives. This study supported these findings and offers two additional strategies as supportive methods that also contribute to a CDO's effort to institutionalize diversity and inclusion. These two strategies are: assessment of the external environment and thorough analysis of the data.

Assessment of the external environment. The first strategy not covered by Williams and Wade-Golden's (2013) research but found in this study is the importance of assessing the external environment to understand how the environment outside of the institution impacts diversity efforts within the university. For example, at Francis University the Provost was cognizant of the fact that enrollment at his campus had reached steady state; he could not grow enrollment or campus facilities due to a land agreement with the city. Yet, in recent years there has been a growing push by trustees and faculty to admit more international students to the campus. As he pointed out, doing 
so would impact the domestic population because he would have to make a deduction in the current enrollment to meet this new demand. He wondered how an effort to move in this direction might impact historically underrepresented minority groups. As a result, he was in regular conversation with his $\mathrm{CDO}$ about the developing desire to admit more international students and its implications to current diversity efforts.

As campuses build up efforts to institutionalize diversity and inclusion they should aware of the impact of the external environment. Furthermore they should think of ways to incorporate the $\mathrm{CDO}$ into upper level decision-making, if that is not already occurring at their campuses. This can help prevent unintended consequences stemming from a decision that may adversely impact diversity. It can also help the $\mathrm{CDO}$ be proactive in making decisions stemming from changes beyond the university's control, such as budget cuts or new legislation. By having the $\mathrm{CDO}$ present during the decisionmaking process the $\mathrm{CDO}$ can be more effective in their job, which helps with ongoing institutionalization efforts.

Thorough analysis of the data. The second strategy found in this study but not articulated by Williams and Wade-Golden's (2013) research was a through analysis of institutional data. Although the authors do discuss the use of data, this is substantially different because the participants in this study applied strategies that required a greater sophistication with the data. In this study the CDOs specified how they worked with the data to understand the current realities of the organization. This included deep dives into the data that looked at specific populations and groups individually. For example, at Central University, an administrator in the CDO office disaggregated large compositional diversity data sets that came form the office of academic and institutional research. The 
diversity administrator disaggregated the data concerning female faculty to get the number of female faculty of color. To her this was very important to understand the true picture of female faculty diversity in the departments. She also disaggregated the data concerning Asian-American faculty, who were unhappy with the fact that their numbers were inflated by including international faculty from countries such as China, Japan, and India. Without the international faculty, the campus had a more accurate number of Asian-American faculty at Central University. The Asian-American faculty members were also able to discuss with the $\mathrm{CDO}$ that their experiences were different from their international colleagues.

This understanding of using the data in a more nuanced and sophisticated manner expands on the Williams and Wade-Golden strategy because it moves beyond the strategy of simply collecting data and reporting it publically. Rather, this strategy underscores the importance and highlights specific practices to be more successful with data. By disaggregating large data sets given by institutional research offices the CDOs could be more strategic with their efforts and zero in on problem areas needing attention. In turn, this has the potential to make the $\mathrm{CDO}$ and the campus more successful in their institutionalization efforts.

\section{Recommendations for Institutional Practice}

A significant goal of this study was to provide data to universities considering hiring a CDO or seeking to make current efforts to institutionalize diversity and inclusion stronger. Therefore, based on the findings and major themes from the study seven important recommendations for institutions to consider have been identified. They are: (1) embed the CDO position within Academic Affairs, (2) hire a faculty CDO with 
tenure, (3) consider a smaller CDO organizational construct, (4) maintain a broad notion of diversity, (5) give special attention to campus climate, (6) assess the external environment, and (7) use a systematic, relational, and integrated approach to advance institutionalization efforts. A discussion of each of the recommendations follows.

\section{CDO Position within Academic Affairs}

A recommendation for campuses considering hiring a $\mathrm{CDO}$ for the first time or for those willing to make changes to an existing CDO structure is to embed the position within Academic Affairs. As this study has shown, this is important because it connects the role of the CDO and their subsequent work to the academic mission of the university. Connecting the work of the CDO to Academic Affairs is significant because it signals that diversity is not only about compositional diversity and reaching targets. Rather diversity and inclusion focused on the academic mission concerns the curriculum and research. It also highlights the benefits of a diverse classroom, which includes faculty and student interactions. It also signals that diversity and inclusion is more than the job of Student Affairs, which has traditionally overseen diversity and multi-cultural programs intended for students. Embedding the CDO in Academic Affairs and connecting their work to the academic mission of the university, identifies diversity and inclusion as a key component to making the institution stronger. It strengthens the concept of diversity and its related benefits to creating more cognitively complex thinkers. This recommendation supports existing research that suggests linking diversity and inclusion to the academic function of the university in order to achieve inclusive excellence (Williams, 2013; Williams, Berger \& McClendon, 2005).

\section{CDO as Tenured Faculty}


The data from this study also supports the recommendation that the CDO be a tenured member of the faculty. This is important because faculty status helped the CDOs achieve buy-in and support from faculty within the various colleges and schools at their universities. The CDOs faculty status and association with the Provosts were important distinctions presented in this study by all three CDOs, Provosts, and faculty interviewed. As one faculty member described, it was extremely important that the CDO be an "academic" connected to the academic function of the university. All three CDOs admitted that being a faculty member gave them entre into one of the toughest groups to work with for institutional change - the faculty. As faculty members, the CDOs were familiar with the pressures newer faculty face to produce research, get tenure, and receive academic promotions. They also were aware that serving on committees or participating in trainings pulled faculty away from research and teaching obligations. To mitigate these pushbacks, the CDOs used the same language as the faculty, cited empirical studies, and spoke in terms of data and evidence-based practices. Furthermore, all three CDOs continued to publish in their fields, two of them in the diversity and higher education field. For the two CDOs who publish in the diversity and higher education field, this further supports their academic credentials among faculty peers and contributes to their credibility when presenting why the campus should be diversity and inclusion-minded.

\section{Smaller Organizational Construct}

Another recommendation to emerge from this study is for campuses to realize that the size of the CDO office does not have to be large or include organizational complexity in order to be effective at leading transformational change. Reorganization of existing offices or units pertaining to diversity to build an entire division does not need to occur. 
This is important to campuses that may be thinking that adding a CDO is a costly endeavor and disruptive to existing departments and offices. In this study, Morrill University and Francis University had small CDO offices. Both CDOs operated within the Collaborative Model (Williams \& Wade-Golden, 2013) and relied on cooperative relationships and lateral coordination. Each $\mathrm{CDO}$ worked in partnership with their fellow vice presidents and associate provosts to accomplish campus-wide diversity goals. This finding may surprise those believing that a large campus, such as Morrill University with over 50,000 students enrolled, should have an equally large CDO office with several diversity and equity units reporting to it. On the contrary, the $\mathrm{CDO}$ at Morrill University preferred a smaller office due to the nimbleness of not having to manage several units that fell under her control. Rather, she and the CDO at Francis University favored the unencumbered ability to work directly with the President and Provost on institutional goals and preferred to use their time more strategically to hold units accountable for their diversity efforts. To be sure, it is hard to imagine being able to conduct both of these large tasks for an entire university with the added responsibility of managing several organizational structures and offices as well.

To support the point that a larger divisional operation is not necessarily the best option, the $\mathrm{CDO}$ at Francis University shared that she was opposed to a larger diversity infrastructure because she wanted to send the message that diversity was everyone's responsibility. She felt that if the CDO's office started to include other diversity and equity related offices, it would send the wrong message that her area was in charge of all things diversity and inclusion for the campus. This would signal that other campus partners no longer had to concern themselves with diversity and inclusion efforts because 
the CDO's unit had the ultimate responsibility for it. Additionally, the CDO office at Morrill University had, at one point, more units reporting to it, but it did not make much organizational sense to the Provost and was causing disruption among other units and faculty. When the $\mathrm{CDO}$ previous to the $\mathrm{CDO}$ in the study left, the $\mathrm{CDO}$ unit was whittled down to its present size to primarily focus on the campus' diversity plan and accountability. While some may argue a smaller CDO office does not have the same stature of other vice presidents, having a smaller CDO office may end up providing the CDO more capacity to lead change because there is less administrative oversight. This in turn may create more opportunity for the CDOs to work collaboratively and build relationships, which in this study proved to be essential in building a critical mass of supporters. Furthermore, should there be organizational change as was the case at Morrill University, a smaller office could better accommodate these changes.

\section{Broad Notion of Diversity}

A further recommendation for campuses to consider is the importance of adopting a broad notion of diversity. As the data from this study was analyzed, it became clear that the campuses considered themselves successful at institutionalizing diversity and inclusion when the campus community possessed a broader understanding of diversity and as a result became diversity-minded. This was due in large part to the intentional efforts of the CDO. A broader understanding signaled a paradigm shift where campuses could consider the positive associations with diversity and inclusion and therefore wanted to include it in their way of thinking, behaving, and acting. Additionally, having a broader notion of diversity allowed for a more sophisticated understanding of the types of diversity that go beyond traditional notions of race. For example, the case study sites 
also embraced diversity related to socio-economic status, military status, ability, and sexual orientation. The campuses understood that a wider definition of diversity made the campus more inclusive and up to date with newer understandings of diversity that exceeded models derived from the $1950 \mathrm{~s}$ and $60 \mathrm{~s}$.

\section{Campus Climate}

As the data in this study showed, campus climate is an important element in a campus' efforts to institutionalize diversity and inclusion. Therefore, a deliberate effort to assess and analyze the campus climate regularly is essential for institutions to implement as they work toward hiring a $\mathrm{CDO}$ and transforming their campuses. In this study, each campus routinely assessed the climate of not only their students, but also their faculty and staff as well. This led to a more comprehensive look at the campus's overarching and culture and support for diversity. In addition, to focusing on the climate for all stakeholder groups, the CDOs in this study paid close attention to groups that were represented in smaller numbers or were traditionally marginalized. Based on the data collected from the climate assessments the CDOs worked with campus partners to develop specific strategies for improving the environment.

It is important to give special attention to a campus' climate in combination with ongoing diversity efforts to get a true sense of the campus environment. Without this close examination campuses may be building diverse institutions but may also be missing a key component that leverages the benefit of diversity beyond counting numbers. $\mathrm{A}$ positive and welcoming campus climate that values the differences amongst its campus community has the potential to accelerate the transformational change.

\section{Assessing the External Environment}


A further recommendation to be considered based on the findings of this study is the assessment of the external environment. The CDOs in this study were active in highlevel discussions that involved campus-wide policy and structural changes. This helped the CDOs be proactive in addressing intended or unintended consequences to a major change impacting the university. As previously mentioned, the campuses in this study were not islands unto themselves and had to monitor outside pressures that would impact current diversity efforts. These external pressures could be from trustees, community members, state legislatures, and even Supreme Court decisions. As a result, the CDOs were in frequent discussion with their Provosts and Presidents about changes coming from the external environment and how they changes could potentially disrupt or assist current diversity and inclusion efforts. Therefore, it is recommended that in addition to concentrating on internal diversity and inclusion efforts, the CDOs and their campuses need to also create space for examining what is coming from the external environment. By integrating this practice into standing meetings or dialogues, campuses and their CDOs could be more effective in their jobs and in protecting the institutionalized efforts they worked so hard to establish.

\section{Systematic, Relational, and Integrated Approach}

Finally, the findings from this study support the recommendation that CDOs intending to institutionalize diversity and inclusion operate with a systematic, relational, and integrated approach. For example, in this study the CDOs worked systematically at building relationships because their efforts relied on how well they could build a critical mass of supporters who could then carry the institutionalization effort into their specific units and departments. Without these relationships the CDOs would have to use their 
positional authority, which is a less effective tool in a decentralized organization like higher education. To support this claim, participants at Morrill University specifically addressed how a top-down approach tried by a previous CDO failed to achieve buy-in. As a result the diversity agenda could not be implemented.

In this study, relationships allowed the CDOs to work intentionally and strategically with campus administrative and academic units to hone in on specific areas needing attention. Their relationships relied on trust built between the CDO and the units on campus. These units trusted that the CDOs would help steer them in the appropriate direction for diversity success. The CDOs aided the departments by using data strategically to point out where the units currently were and where they needed to be. This included deep dives into the data and disaggregating large data sets to get a truer picture of areas needing improvement. Overtime the CDOs were able to integrate the multiple diversity efforts taking place within the institutions into one cohesive movement leading to transformational change. The strategy of working in an systematic, relational, and integrated approach should be implemented at campuses looking to institutionalize diversity and inclusion because of the demonstrated successes the CDOs and campuses in this study.

\section{Recommendations for Future Research}

From this study there are implications for future research. Three areas in particular should be considered. They are: research on institutionalization efforts without a CDO, research utilizing leadership theory to understand the various ways CDOs lead change efforts, and research that includes external stakeholders to validate the diversity efforts of the university and that also considers the role of the external community in 
institutionalization efforts. These areas in combination with the data that emerged from this study can add to the literature on CDOs in higher education. A more thorough discussion on each follows.

\section{Institutionalization Efforts Without a CDO}

This study could be replicated to examine universities that have institutionalized diversity and inclusion without the assistance of a CDO. It would be beneficial to know what strategies these campuses used, what other institutional leaders were involved, and what was their role in advancing the institutionalization effort. This is important to study because not every university will have the resources or institutional capacity to hire a CDO or create a diversity office such as the ones in this study. In their latest research Williams and Wade-Golden (2013) compared universities with a CDO to those without a $\mathrm{CDO}$ to measure the differences in the priority of diversity. Their study looked at the intensity of priority as it relates to strategic diversity planning systems, accountability systems, research and assessment systems, training and education initiatives, and faculty diversification efforts. Overall they found that institutions with a CDO had more robust diversity efforts in place than those who did not. Although useful, this examination does not fully address the many associated questions related to an effort to institutionalize diversity and inclusion with a CDO. It only makes the argument that a CDO is a helpful position in doing so; it does not help universities understand the ways in which to go about leading an institutionalization effort without a CDO.

More empirical studies need to be conducted that go beyond Williams and WadeGolden's (2013) comparison measurers. These studies will help to provide a clearer understanding of the comprehensive role of CDOs in institutionalizing diversity and 
inclusion. This particular study chose to focus on successful campuses with a CDO because more information on how CDOs contribute to the institutionalization of diversity and inclusion was desired. Results from this study combined with similar studies without a CDO may offer a more complete picture of what it truly takes to have a successful institutionalization effort.

\section{Leadership Theory and CDOs}

A second area where further research could be conducted concerns CDOs and their leadership style. Throughout this study, several participants, including all of the CDOs, indicated the work of the CDO is to bring others along in fulfilling the campus' diversity and inclusion goals. Moreover, they each emphasized the importance of "buyin" to achieve a critical mass of diversity supporters that could carry the institutionalization effort into their respective departments and areas. While this study identified specific ways in which the CDOs achieved buy-in and support, it did not examine the specific leadership methods or styles of the CDOs, using existing leadership theories as a framework. Herein lies a great opportunity to understand the complexity of the CDO's role and position that moves beyond an analysis of how it is operationalized. As a result of this study and others like it, few could argue leadership and leadership skills are not essential to the role of CDOs. Thus, more phenomenological studies using leadership theories combined with studies such as this one are needed to get at the root of how the CDOs operate in their organizational contexts. Otherwise, if the literature is only presented in a manner of themes, steps, or best practices, this will oversimplify the job of the $\mathrm{CDO}$ at leading transformational change. Additionally, it may also diminish the critical components identified in this study associated with the successful results for 
institutionalizing diversity and inclusion. Furthermore, the body of literature focusing on leadership in higher education needs to also include studies on CDOs. Currently, the majority of leadership studies and higher education focuses on presidents and chief academic officers and does not consider the emerging field of CDOs nor their responsibility for promoting diversity and inclusion throughout the entire higher education organization.

\section{External Stakeholders}

A third area for further research is the consideration of external stakeholders and how they perceive a campus' efforts to institutionalize diversity and inclusion. In this research study only internal campus stakeholders were interviewed because they tend to be the most knowledgeable and actively engaged in the effort to institutionalize diversity and inclusion. It is important to study stakeholders outside the campus, such as alumni and community partners to get their perspective on the campus' efforts to be more inclusive. As one campus in the study noted, alumni play a large and influential role in assisting the campus in its diversity efforts and at other times can oppose steps to be more welcoming and inclusive. Another campus noted that a large population of their alumni remain in the state and can influence decisions the campus makes politically. Furthermore, Morrill University had alumni and community members serve on some its leadership committees concerning diversity. They felt it essential to include community members to bolster support and to improve town-gown relations.

Future studies involving efforts to institutionalize diversity should consider stakeholder groups outside of the campus because many, such as this study, tend to focus on actions within the university but fail to demonstrate the impact or effect of diversity 
efforts outside of the university. Additionally, by considering outside stakeholders more could be learned about how campus diversity efforts intersect with external groups such as employers or even with legislators. Furthermore, involving outside stakeholders reflects a more accurate depiction of reality because universities do not operate in isolation. Rather, they are influenced by the external context and environment in which they operate.

In conclusion this study offers an initial step toward future research in this area. An understanding of the many ways campuses with a CDO successfully institutionalize diversity and inclusion has begun. From this study, additional aspects of leading transformational change efforts in support of diversity and inclusion should be explored. Future studies that examine institutionalization efforts without a CDO, the importance of leadership, and the perspectives and contributions of external stakeholders should be conducted. It is hoped that the findings presented in this study will help future research endeavors that will build upon the literature focusing on CDOs in higher education.

\section{Final Summary}

This multiple case study focused on the role of the $\mathrm{CDO}$ at three exemplar universities specifically known to be succeeding in their efforts to institutionalize diversity and inclusion campus-wide. Examining institutions that have experienced success, as determined by fellow CDO peers, offers more opportunity to learn how CDOs institutionalize diversity and inclusion. This study suggests that universities can institutionalize diversity and inclusion with the efforts of a $\mathrm{CDO}$ who is committed to leading a transformational change process that is supported by a systematic, integrated, and relational approach. Specifically, what is needed is change that moves diversity from 
the margins of the institution, where it is traditionally isolated and relegated to a minor role, to the center of the university where it is intentionally connected to the mission of the university and part of the university's collective consciousness.

Through intentional efforts that include relationship building, trust, and patience for a long change process, CDOs can help colleges and universities institutionalize diversity and inclusion so that it is a stable and permanent condition of the university that positively affects the way a university functions and makes decisions. These efforts led by the CDO rely on strategies that bring campus stakeholders into the change process wherever they may be in their understanding of diversity, so that overtime diversity supporters and advocates increase and the diversity and inclusion effort has a greater opportunity to permeate the wider organization. In this sense, diversity becomes more than an annual exercise, task of compliance, or one person's job; rather, diversity and inclusion become part of a university's psyche upheld by a critical mass of supporters.

In this study, the CDOs used various strategies to sustain their diversity efforts and to receive greater support and acceptance for diversity work. This study suggests that when campuses have a broader understanding of diversity that moves beyond traditional notions of race and affirmative action, the campuses are better poised to link the importance of diversity and inclusion to the mission of the university. This means that campuses will have to develop a more sophisticated and better understanding of diversity that exceeds traditional measures of counting the quantity of diverse people in the organization or solely focuses on factors related to student success of historically underrepresented groups. Furthermore, campuses will need to change the culture of the university. It will be imperative to address campus climate along with diversity to 
understand the way campus community members feel at their institutions. CDOs are likely to be better positioned to effect change by having a more accurate picture of what is taking place at their institutions and outside of the confines of the institutions, so they are more able to address what needs to be changed and sustained. These efforts are supported by the integral work of relationships and collaboration led by the CDO. Without the relationships the CDOs work becomes significantly hampered.

Finally, this study revealed five specific strategies successful campuses with a CDO utilize to institutionalize diversity and inclusion. This is helpful to campuses considering hiring a CDO because they will need to identify ways to sustain their efforts and to build greater buy-in. Working without resources that can guide a CDO's efforts could be problematic and possibly lead to failed change efforts that are intended to support diversity and inclusion. By using the findings from this study, CDOs may be able to help their campuses institutionalize diversity and inclusion in a more efficient and successful manner that will lead to transformational change. 


\section{References}

Alger, J. R. (1997). The educational value of diversity. Academe, 83(1), 20-23.

Altbach, P.G (2011). Patterns of higher education development. In Altbach, P., Gumport, P., \& Berdahl, R. (Eds.), American higher education in the twenty-first century: Social, political, and economic challenges. (pp. 15-36). Baltimore, Maryland: John Hopkins University Press.

Altbach, P. G., Gumport, P. J., \& Berdahl, R. O. (Eds.). (2011). American higher education in the twenty-first century: Social, political, and economic challenges. Baltimore, MD: John Hopkins University Press.

Antonio, A. L., Chang, M. J., Hakuta, K., Kenny, D. A., Levin, S., \& Milem, J. F. (2004). Effects of racial diversity on complex thinking in college students. Psychological Science, 15(8), 507-510.

Argyris, C. (1994). Good communication that blocks learning. Harvard Business Review, 72(4), 77-85.

Argyris, C., \& Schon, D. (1978). Organizational learning: A theory of action perspective. Reading, MA: Addison-Wesley.

Astin, A. W. (1993). Diversity and multiculturalism on the campus: How are students affected? Change: The Magazine of Higher Learning, 25(2), 44-49.

Baldridge, V. (1980). Organizational characteristics of colleges and universiteis. In Baldridge, V. \& Deal, T. (Eds.), The dynamics of organizational change in education. (p. 38-59). Berkeley, CA: McCutchan Publishing Corp.

Barceló, N. (2007). Transforming out institutions for the twenty-first century: The role of the chief diversity officer. Diversity Digest. 10(2), 5-6. 
Banerji, S. (2005). Diversity officers coming to a campus near you? Diverse Issues in Higher Education, 22(20), 38-40.

Bensimon, E. M. (2004). The diversity scorecard: A learning approach to institutional change. Change: The magazine of higher learning, 36(1), 44-52.

Bensimon, E.M., Neumann, A., \& Birnbaum, R. (1989). Making sense of administrative leadership: The "L" word in higher education. San Francisco, CA: Jossey-Bass.

Bergquist, W.H. (1992). The four cultures of the academy. San Francisco, CA: JosseyBass.

Bess, J.L., \& Dee, J.R. (2008). Understanding college and university organization: The state of the system (Vol. 1). Sterling, VA: Stylus Publications

Birnbaum, R. (1988). How colleges work the cybernetics of academic organization and leadership. San Francisco, CA: Jossey-Bass Publishers.

Birnbaum, R. (1989). The cybernetic institution: Toward an integration of governance theories. Higher Education, 18(2), 239-253. doi: 10.1007/BF00139183

Bolman, L.G., \& Deal, T. E. (1997). Reframing organizations: Artistry, choice and leadership ( $2^{\text {nd }}$ ed.). San Francisco, CA: Josey Bass Publishers.

Boyce, M.E. (2003). Organizational learning is essential to achieving and sustaining change in higher education. Innovative Higher Education, 28(2), 119-136.

Chang, M. J. (1999). Does racial diversity matter?: The educational impact of a racially diverse undergraduate population. Journal of College Student Development, 40(4), $377-395$.

Chang, M.J. (2002). Preservation of transformation: Where's the real educational discourse on diversity? The Review of Higher Education, 25(2), 125-140. 
Charmaz, K. (2006). Constructing grounded theory: A practical guide through qualitative research. Thousand Oaks, CA: Sage Publications.

Christensen, C. M., \& Eyring, H. J. (2011). The innovative university: Changing the DNA of higher education from the inside out. San Francisco, CA: Jossey-Bass.

Clark, B.R. (1983). The contradictions of change in the academic system. Higher Education, 12(1), 101-116. (EJ 279 751).

Clark, C., Fasching-Varner, K., \& Brimhall-Vargas, M (Eds.) Occupying the academy. Just how important is diversity work in highger education. Lanham, MD: Rowman \& Littlefied Publishers, Inc.

Clayton-Pedersen, A., Parker, S., Smth, D., Moreno, J., \& Hiroyuki Teraguchi, D. (2007). Making a real difference with diversity: A guide to instiutional change. Washington, DC: Association of American Colleges \& Universities.

Cohen, M., \& March, J. (1974). Leadership and organized anarchy. In Baldridge, V. \& Deal, T. (Eds.), The dynamics of organizational change in education. (pp. 333366). Berkeley, CA: McCutchan Publishing Corp.

College Board (2012). Trends in college pricing 2012. Retrieved on July 7, 2014 from http://trends.collegeboard.org/sites/default/files/college-pricing-2012-full-report121203.pdf

Curry, B. K. (1992). Instituting enduring innovations: achieving continuity of change in higher education. ASHE-ERIC Higher Education Report No.7. Washington, DC: ASHE-ERIC Higher Education Reports.

Davis, L.R. (2002). Racial diversity in higher education: Ingredients for success and failure. The Journal of Applied Behavioral Science, 38(2), 137-156. 
DiRamio, D., Ackerman, R., \& Mitchell, R. L. (2008). From combat to campus: Voices of student-veterans. NASPA Journal, 45(1).

Eckel, P. D., \& Kezar, A. J. (2003). Taking the reins: Institutional transformation in higher education. Westport, CT: Greenwood Publishing Group

Glaser, B.G., \& Strauss, A.L. (1967). The discovery of grounded theory. Chicago, IL: Aldine.

Glesne, C. (2006). Becoming qualitative researchers: An introduction ( $3^{\text {rd }}$ ed.). Boston, MA: Pearson Education, Inc.

Greenwood, R., \& Hinings, C. R. (1996). Understanding radical organizational change: Bringing together the old and the new institutionalism. Academy of management review, 21(4), 1022-1054.

Gose, B. (2013, June, 9). Diversity offices aren't what they used to be. The Chronicle of Higher Education. Retrieved on July 7, 2014 from http://chronicle.com/article/Diversity-Offices-Arent-What/139633

Gose, B. (2006, Sept., 29). The rise of the chief diversity officer. The Chronicle of Higher Education. Retrieved on July 7, 2014 from http://chronicle.com/article/The-Riseof-the-Chief/7327

Gurin, P. (1999). Export report of Patricia Gurin. Selection from the compelling need for diversity in higher education, expert reports in defense of the University of Michigan. Equity \& Excellence in Education. 32:2, (pp. 36-62).

Gurin, P., Dey, E., Hurtado, S., \& Gurin, G. (2002). Diversity and higher education: Theory and impact on educational outcomes. Harvard Educational Review, 72, (3), 330-336. 
Harper, S. R., \& Antonio, A. L. (2008). Not by accident: Intentionality in diversity, learning, and engagement. In S. R. Harper (Ed.), Creating inclusive campus environments for cross-cultural learning and student engagement, (pp. 1-18). Washington, DC: NASPA

Harper, S. R., \& Hurtado, S. (2007). Nine themes in campus racial climates and implications for institutional transformation. New Directions for Student Services, (120), 7-24.

Hurtado, S. (2007). Linking diversity with the educational and civic missions of higher education. The Review of Higher Education, 30(2), 185-196.

Hurtado, S., \& Carter, D. F. (1997). Effects of college transition and perceptions of the campus racial climate on Latino college students' sense of belonging. Sociology of Education, 324-345.

Hurtado, S., Milem, J., Clayton-Pedersen, A, \& Allen, W. (1999). Enacting diverse learning environments: Improving the climate for racial/ethnic diversity in higher education. ASHE-ERIC Higher Education Report, 26, (8). Washington, DC: ERIC Clearinghouse on Higher Education.

Kezar, A. J. (2007). Tools for a time and place: Phased leadership strategies to institutionalize a diversity agenda. The Review of Higher Education, 30(4), 413439.

Kezar, A.J. (2001). Understanding and facilitating organizational change in the 21st Century: Recent research and conceptualizations. San Francisco: Jossey-Bass Publishers. 
Kezar, A. J., \& Eckel, P. D. (2004). Meeting today's governance challenges: A synthesis of the literature and examination of a future agenda for scholarship. The Journal of Higher Education, 75(4), 371-399.

Kezar, A.J., \& Eckel, P.D. (2002). The effect of institutional culture on change strategies in higher education: Universal principles or culturally responsive concepts? The Journal of Higher Education, 73(4), 435-460.

Kotter, J.P. (1996). Leading change. Boston, MA: Harvard Business Press.

Kramer, M. (2000). Making it last forever: The institutionalizing of service learning. Wahsington, DC. Corportation for National Service.

Kuhn, T. S. (2012). The structure of scientific revolutions. Chicago, IL: University of Chicago Press.

Kvale, S., \& Brinkmann, S. (2009). Interviews: Learning the craft of qualitative research interviewing (2nd ed.). Thousand Oaks, CA: Sage Publications, Inc.

LeCompte, M. D., Preissle, J., \& Tesch, R. (1993). Ethnography and qualitative design in educational research. $\left(2^{\text {nd }}\right.$ ed.) Orlando, FA: Academic Press.

Leon, R.A. (2014). The chief diversity officer: An examination of CDO Models and Strategies. Journal of Diversity in Higher Education, 7(2), 77-91.

Lueddeke, G.R. (1999). Toward a constructivist framework for guiding change and innovation in higher education. The Journal of Higher Education, 70(3), 235-260. doi: $10.2307 / 2649196$

Merriam, S. B. (1998). Qualitative Research and Case Study Applications in Education. San Francisco, CA: Jossey-Bass Publishers.

Journal of Diversity in Higher Education (2014). 
Milem, J. F. (2003). The educational benefits of diversity: Evidence from multiple sectors. In Chang, M.J., Witt, D., Jones, J., \& Hakuta, K. (Eds.), Compelling interest: Examining the evidence on racial dynamics in higher education (pp. 12169). Stanford, CA: Stanford University Press.

Milem, J.F., Chang, M.J., Antonio, A.L. (2005). Making diversity work on campus: A research based perspective. Washington, DC: Association of American Colleges \& Universities.

Mottel, S. \& Patten, E. (2013). Statistical report of Hispanics in the United States, 2011. Washington, DC: Pew Research Center.

National Association of Diversity Officers in Higher Education (n.d.). History. Retrieved on July 7, 2014 from http://www.nadohe.org/history

National Association of Diversity Officers in Higher Education (n.d.). President's Message. Retrieved on July 7, 2014 from ttp://www.nadohe.org/nadohepresidents-message

National Association of Diversity Officers in Higher Education (n.d.). Vision and mission statements. Retrieved on July 7, 2014 from http://www.nadohe.org/vision-amission-statements

Office of Post Secondary Education, US Department of Education (n.d.). Welcome to the office of post secondary education home page. Retrieved on July 7, 2014 from http://www2.ed.gov/about/offices/list/ope/index.html.

Passel, J. S. (2006). The size and characteristics of the unauthorized migrant population in the U.S. Washington, DC: Pew Hispanic Center. Retrieved on July 7, 2014 from http://pewhispanic.org/files/reports/61.pdf. 
Patton, M. Q. (2002). Qualitative research and evaluation methods. Thousand Oaks, CA: Sage Publications, Inc.

Regents of the University of California vs. Bakke 438 US 312, 98 S. Ct. 2760 (1978).

Rendón, L. I. (1994). Validating culturally diverse students: Toward a new model of learning and student development. Innovative higher education, 19(1), 33-51.

Santiago, D. \& Calderón Galdeano, E. (2014). Latino college completion: United States. Washington, DC: Excelencia in Education

Saldaña, J. (2009). The coding manual for qualitative researchers. Thousand Oaks, CA: Sage Publications Inc.

Schein, E.H. (2004). Organizational culture and leadership. ( $3^{\text {rd }}$ ed.). San Francisco, CA: Jossey-Bass.

Senge, P.M. (1990). The fifth discipline: The art and practice of the learning organization. New York, NY: Doubleday Currency.

Simsek, H., \& Louis, K.S. (1994). Organizational change as paradigm shift: Analysis of the change process in a large, public university. The Journal of Higher Education, $670-695$.

Smith, D. G. (1997). How diversity influences learning. Liberal Education 83(2): $42-7$

Smith, D. G. (2009). Diversity's promise for higher education. Baltimore, MD: John Hopkins University Press.

Sporn, B. (1999). Adaptive university structures: An analysis of adaptation to socioeconomic environments of US and European universities. London, UK: Jessica Kingsley. 
Terenzini, P. T., Cabrera, A. F., Colbeck, C. L., Bjorklund, S. A., \& Parente, J. M. (2001). Racial and ethnic diversity in the classroom: Does it promote student learning? Journal of Higher Education, 72 (5), 509-531.

Tierney, W.G. (1988). Organizational culture in higher education: Defining the essentials. The Journal of Higher Education, 15, (1), 2-21.

Trowler, P. (2008). Cultures and change in higher education: Theories and practices. New York, NY: Palgrave Macmillan.

Weick, K. (1978). Educational organizations as loosely coupled systems. In Baldridge, V. \& Deal, T. (Eds.), The dynamics of organizational change in education. (pp. 15-38). Berkeley, CA: McCutchan Publishing Corp.

Williams, D.A. (2008). Beyond the diversity crisis model: Decentralized diversity planning and implementation. Planning for Higher Education, 36(2), 27-41.

Williams, D. A. (2013). Strategic diversity leadership: Activating change and transformation in higher education. Sterling, VA: Stylus Publishing.

Williams, D.A., Berger, J.B., \& McClendon, S.A. (2005). Toward a model of inclusive excellence and change in postsecondary institutions. Washington, DC: Association of American Colleges and Universities.

Williams, D. A., \& Clowney, C. (2007). Strategic planning for diversity: A primer for academic leaders. Effective Practices for Academic Leaders, 2(3), 1-16.

Williams, D.A. \& Wade-Golden, K. (2008). The chief diversity officer: A primer for college and university presidents. Washington, DC: American Council of Education. 
Williams, D. A., \& Wade-Golden, K. (2013). The chief diversity officer: Strategy structure, and change management. Sterling, VA: Stylus Publishing.

Williams, D.A. \& Wade-Golden, K. (2006, April, 16). What is a chief diversity officer? Inside Higher Education. Retrieved on July 7, 2014 from http://www.scribd.com/dawilliams3/d/14860557--What-is-a-Chief-DiversityOfficer-by-Dr-Damon-A-Williams-and-Dr-Katrina-WadeGolden

Yin, R. K. (2005). Case study research: design and methods ( $3^{\text {rd }}$ ed.). Thousand Oaks, CA: Sage Publications.

Yosso, T. J. (2006). Critical race counterstories along the Chicana/Chicano educational pipeline. New York, NY: Rutledge.

Zaltman, G., \& Duncan, R. (1977). Strategies for planned change. New York, NY: John Wiley. 
Appendix A

Members of NADOHE at the Time of Study 
Members of the National Association of Diversity Officers in Higher Education (NADOHE) at the time the questionnaire was administered

Alcorn State University

Allegheny College

Anna Maria College

Arizona State University

Arkansas State University - Jonesboro

Association of American Medical Colleges

Babson College

Berklee College of Music

Buffalo State College

California Polytechnic State University

California State University San Marcos

California State University, Fresno

California University of Pennsylvania

Case Western Reserve University

Colgate University

College of Charleston

College of Southern Nevada

College of the Holy Cross

College of William \& Mary

Colorado State University

Cornell University

Dartmouth College

DePaul University

Des Moines University

Duke University

East Carolina University

Eastern Virginia Medical School

Fashion Institute of Technology

Gadsden State Community College

Gallaudet University

George Mason University

George Washington University

Georgetown University

Georgia Institute of Technology

Georgia State University

Gonzaga University

Grand Valley State University

Grinnell College

Hamilton College

Harvard University

Henderson State University

Hillsborough Community College

Hopkinsville Community College 
Houston Community College

ILIFF School of Theology

Indiana State University

Indiana University

Iowa State University

Ivy Tech Community College

James Madison University

Kennesaw State University

Kent State University

Lehigh University

Metropolitan Community College-Kansas City

Miami University

Michigan State University

Middle Tennessee State University

Middlebury College

Milwaukee Area Technical College

Minnesota State Colleges and Universities

Minnesota State University, Mankato

Mississippi State University

Missouri State University

Montgomery College

National Collegiate Athletic Association (NCAA)

North Carolina State University at Raleigh

North Dakota State University

Northern Arizona University

Oakton Community College

Oklahoma State University

Paradise Valley Community College

Penn State University Altoona

Pennsylvania College of Technology

Point Loma Nazarene University

Portland Community College

Portland State University

Princeton University

Providence College

Purdue University

Rice University

Rochester Institute of Technology

Samuel Merritt University

Savannah State University

Smith College

Southeast Community College

Southern Illinois University Edwardsville

St. John Fisher College

St. Joseph's University

St. Olaf College 
Stanford University

State University of New York at Albany (main campus)

State University of New York, Albany

STONY BROOK UNIVERSITY

Susquehanna University

Syracuse University

Tarrant County College District

Tennessee Board of Regents

Tennessee State University

Texas A\&M University

Texas Tech University

The College at Brockport

The Ohio State University

The Pennsylvania State University

The Richard Stockton College of New Jersey

The University of Akron

The University of North Carolina at Greensboro

The University of Wisconsin Milwaukee

Tiffin University

Towson University

Truckee Meadows Community College

Tulane University

Union College

University of Arizona

University of California, Los Angeles

University of California-Berkeley

University of California-Davis

University of California-San Diego

University of Central Florida

University of Colorado at Colorado Springs

University of Connecticut

University of Delaware

University of Denver

University of Florida

University of Georgia

University of Houston

University of Idaho

University of Iowa

University of Kansas

University of Kentucky

University of La Verne

University of Louisville

University of Maryland

University of Maryland University College

University of Massachusetts Boston

University of Missouri 
University of Nebraska-Lincoln

University of Nevada, Las Vegas

University of New Hampshire

University of New Mexico

University of North Alabama

University of North Carolina at Chapel Hill

University of North Carolina Wilmington

University of North Texas

University of Oregon

University of Puget Sound

University of Rochester

University of Tennessee

University of Texas at Arlington

University of Texas at Austin

University of Texas at Dallas

University of the Rockies

University of Virginia

University of Washington

University of Wisconsin-Madison

University of Wisconsin-River Falls

Valdosta State University

Valencia College

Vanderbilt University

Villanova University

Virginia Commonwealth University

Virginia Tech University

Wake Forest University

Washington State University

West Virginia University

Western Kentucky University

Western Michigan University

Wheelock College

Winston Salem State University

Wright State University

Xavier University 
Appendix B

NADOHE Questionnaire 


\section{Questionnaire to members of NADOHE}

1. Are you currently a chief diversity officer in a college or university within the United States? For purposes of this study a chief diversity is defined as someone who is the face of diversity efforts at the campus-wide level and who is a senior administrator that is member of the president's senior cabinet.

a. Yes

b. No - If no, this concludes the survey. Thank you for your participation.

2. What is your official chief diversity officer position title, e.g. Vice President/Provost for Diversity, etc.?

3. Do you hold a joint appointment in the faculty?
a. Yes
b. No

4. Beside a faculty appointment, do you hold other positions in the university?
a. Yes
b. No
c. If yes, what other positions do you hold?

5. Who do you report to in the university?
a. President
b. Provost/Executive Vice President
c. Other:

6. Which are you a member of?
a. President's senior cabinet
b. Provost's senior cabinet
c. Other:

7. Where is your position organizationally located within the university?
a. President's Office
b. Academic Affairs
c. Student Affairs
d. Other

8. How many direct reports do you have?
a. $5+$
b. 4
c. 3
d. 2
e. 1
f. None

9. Please name the departments/units that report to you? 
10. What is your annual operating budget?
a. Less than $\$ 100 \mathrm{~K}$
b. $\$ 100 \mathrm{~K}-\$ 200 \mathrm{~K}$
c. $\$ 200 \mathrm{~K}-\$ 300 \mathrm{~K}$
d. $\$ 300 \mathrm{~K}-\$ 400 \mathrm{~K}$
e. $\$ 400 \mathrm{~K}-\$ 500 \mathrm{~K}$
f. $\$ 500 \mathrm{~K}+$

11. In two to three sentences, please describe your position.

12. In your professional opinion as a chief diversity officer, please identify two colleges or universities you believe have been successful at institutionalizing diversity or are close to achieving that goal. (Please list full name of the college or university. No acronyms.)

a.

b.

13. Based on your answer to question $12 \mathrm{a}$, please select no more than five metrics that support your choice.

- Compositional diversity among the students body (undergraduate and graduate)

- Compositional diversity among faculty

- Compositional diversity among staff

- Diverse curricula (majors, minors, GE requirements)

- Equitable retention and graduation rates for underrepresented minority students

- Institutional policies that promote diversity

- Institutional mission statement that delineates the type of diversity the campus is striving for and includes language that also addresses inclusion and equity

- A president that champions diversity publicly

- A chief diversity officer

- A campus-wide strategic plan for diversity

- Support and buy-in from the local community on diversity initiatives

- Other metric not listed:

14. Based on your answer to question $12 \mathrm{~b}$, please select no more than five metrics that support your choice.

- Compositional diversity among the students body (undergraduate and graduate)

- Compositional diversity among faculty

- Compositional diversity among staff

- Diverse curricula (majors, minors, GE requirements)

- Equitable retention and graduation rates for underrepresented minority students 
- Institutional policies that promote diversity

- Institutional mission statement that delineates the type of diversity the campus is striving for and includes language that also addresses inclusion and equity

- A president that champions diversity publicly

- A chief diversity officer

- A campus-wide strategic plan for diversity

- Support and buy-in from the local community on diversity initiatives

- Other metric not listed:

15. Is there anything else you would like to share about your position that was not asked in this brief survey?

Thank you. 
Appendix $\mathrm{C}$

Questionnaire Findings 
As described in Chapter 3, the questionnaire served two purposes for this study. The first was to gain information on the role of CDOs in American institutions of higher education. The second was to identify the case study sites. The questionnaire was administered two separate times to current members of NADOHE in the fall of 2013. It was sent out both times to two NADOHE managed listservs. The first listserv contained 144 email addresses of current members. The second listserv consisted of 55 emails of individuals who self-identified as CDOs and opted into this position specific list. Of this total, 39 respondents completed the short questionnaire, although all 39 respondents did not answer every question. A total of 33 respondents identified themselves as CDOs and moved on to answer the remaining fourteen questions. Six respondents indicated they were not CDOs and were instructed not to complete the questionnaire. Of the 33 respondents who completed the survey, the majority indicated that they were either a vice presidents/provosts or were assistant vice presidents/provosts at their institutions. Some of the respondents who did not fall into this category were advisors to the president for diversity or affirmative action. Titles within the universities varied depending on the name of the units the CDO led; however, the majority contained the word diversity. A few examples included vice president or assistant vice president of: Access, Equity, and Diversity; Diversity and Inclusion; Diversity and Campus Climate; Human Resources, Diversity and Inclusion; Faculty Development and Inclusive Excellence; Institutional Equity to name a few. The respondents were almost evenly split between CDOs from public and private universities. All were from four-year degree granting institutions. From the sample that completed the questionnaire, $20 \%$ had a joint appointment as a faculty member at their institution; however, the affiliated departments varied and 
showed no commonality. Other than faculty appointments, two CDOs indicated that they were an ombudsperson for their campuses and another two indicated that they were the campus' Title IX coordinator.

Fifty-three of the respondents indicated that they report directly to the president of the university, while $23 \%$ report to the provost or executive vice president. Similarly, the majority of the respondents shared they were a member of the president's senior cabinet. Another group reported that they were members of both the president's and the provost's cabinets. Sixty percent indicated that they reported directly to the president's office, while $23 \%$ indicated that they reported to Academic Affairs. The remaining respondents indicated that they reported to Student Affairs or another administrative department such as, Human Resources. Table 3 details the results. Similarities in organizational location and reporting lines ended when it came to department size, operating budgets, and units reporting to the CDOs. The majority of respondents (26.67\%) indicated that they had $5+$ employees reporting directly to the $\mathrm{CDO}$; while the next largest group of respondents $(23.33 \%)$ shared they only had one person report to them. The next largest percentage of direct reports was two employees and no employees; hence the median number of employees reporting to the $\mathrm{CDO}$ in this study was two. The units reporting to the CDO ranged from the multicultural center, access and disability services; international student services; office of equity and pluralism to the African-American cultural center; lesbian, gay, bisexual, transgender resource center; and the ombudsmen's office. The vast majority of respondents indicated that they had a budget of less than $\$ 100,000$, while the next largest response garnered $18.5 \%$ with budgets between $\$ 100,000$ and $\$ 200,000$. Only $11 \%$ indicated budgets of $\$ 500,000$ or more. It must be noted; however, these 
numbers may or may not reflect salaries, as the term "operating budget" was not defined for those responding to the questionnaire. Respondents could have or might not have included their own or employees salaries in this figure.

Table 3.

CDO Reporting Line

\begin{tabular}{|cc|}
\hline CDO & Response Percentage \\
Reporting Line & $60 \%$ \\
President's Office & $23.33 \%$ \\
Academic Affairs & $6.67 \%$ \\
Student Affairs & $10 \%$ \\
Other & \\
\hline
\end{tabular}

The findings from this questionnaire generally support the literature most notably outlined by Damon A. Williams and his associates. While there are some consistencies among the position such as administrative titles, reporting line, and membership in leadership cabinets, there are also a great deal of differences as is demonstrated by this brief questionnaire. For example there is variation among the units the $\mathrm{CDO}$ oversees, the employees who report to the $\mathrm{CDO}$, and it's operating budgets. All together the data returned from the questionnaire provides a context and a picture of the role CDOs play within higher education, albeit with a fairly small sample size. However, the findings shed light on the intricacies of the position and its stature within the overall structure of the university, most notably as a right-hand advisor to the senior leadership of the university. A deeper diver within the case study sites reveals more nuanced information about the $\mathrm{CDO}$ and in particular their role in campus-wide institutionalization efforts. This closer look is discussed in Chapter 4 and divided among the three case study institutions. 
As indicated earlier, the questionnaire served two purposes for this study. The second purpose was to identify the three universities for the case studies. The last group of questions on the survey asked respondents to use their professional judgment to identify two universities that they considered to have successfully institutionalized diversity or are close to reaching that goal. Twenty-six individuals responded to this question yielding 49 responses, which resulted in a list of 39 unique institutions listed. The breakdown of these institutions and their characteristics can be found in Chapter 3 in the methodology section describing the selection of the three case study sites. However, it is important to list the reasons why the 39 institutions were selected and the metrics the CDOs responding to this questionnaire used to make their selection. The breakdown is listed in Table 4.

\section{Table 4.}

Reasons for Selecting a Campus that has Institutionalized Diversity or is close to Reaching that Goal.*

\begin{tabular}{|cc|}
\hline Response & Response Percentage \\
\cline { 2 - 2 } Institutional Policies & $30.44 \%$ \\
Chief Diversity Officer & $29.04 \%$ \\
Strategic Plan for Diversity & $23.9 \%$ \\
Student Diversity & $23.8 \%$ \\
Diverse Curricula & $22.65 \%$ \\
Faculty Diversity & $19.8 \%$ \\
Equitable Graduation \& Retention Rates & $15.85 \%$ \\
President Champions Diversity & $13.2 \%$ \\
Staff Diversity & $9.31 \%$ \\
Institutional Mission Statement & $9.31 \%$ \\
Community Support & $1.35 \%$ \\
Other & $1.35 \%$ \\
\hline
\end{tabular}

* Respondents were instructed to select two campuses. 
Appendix D

CDO/Diversity Offices Websites 
CDO/Diversity Office Website Francis University

\begin{tabular}{|c|c|c|c|c|}
\hline $\begin{array}{l}\text { Title of the } \\
\text { CDO } \\
\text { Report Line }\end{array}$ & $\begin{array}{c}\text { Statement on } \\
\text { what the CDO } \\
\text { Office Does }\end{array}$ & $\begin{array}{c}\text { Diversity } \\
\text { Strategic Plan }\end{array}$ & $\begin{array}{c}\text { Organizational } \\
\text { Chart }\end{array}$ & Definitions \\
\hline \begin{tabular}{|l|} 
Vice President \\
for Intercultural \\
Affairs \\
Report to the \\
President - \\
close working \\
relationship \\
with the \\
Provost - in his \\
academic suite \\
\end{tabular} & $\begin{array}{l}\text { Yes } \\
\text { No info on the } \\
\text { CDO } \\
\text { specifically }\end{array}$ & $\begin{array}{l}\text { Yes } \\
\text { Provides links } \\
\text { to reports, work } \\
\text { plans, updates }\end{array}$ & $\begin{array}{l}\text { No but list the } \\
\text { staff make-up } \\
\text { of the office }\end{array}$ & $\begin{array}{l}\text { No but } \\
\text { documents } \\
\text { within the } \\
\text { strategic plan } \\
\text { do define the } \\
\text { campus } \\
\text { approach and } \\
\text { model }\end{array}$ \\
\hline $\begin{array}{c}\text { Programs \& } \\
\text { Services }\end{array}$ & $\begin{array}{c}\text { Letter/ } \\
\text { Statement } \\
\text { from Provost - } \\
\text { President }\end{array}$ & Resources & $\begin{array}{c}\text { Campus } \\
\text { Statistics / } \\
\text { Campus } \\
\text { Studies } \\
\text { Findings } \\
\end{array}$ & Other \\
\hline $\begin{array}{l}\text { Divided into } \\
\text { faculty, staff, } \\
\text { students, } \\
\text { external } \\
\text { community - } \\
\text { lists pre- } \\
\text { existing efforts/ } \\
\text { initiatives }\end{array}$ & Yes & $\begin{array}{l}\text { President's } \\
\text { Leadership } \\
\text { Initiative - } \\
\text { Faculty } \\
\text { Professional } \\
\text { Development } \\
\\
\text { Inclusive } \\
\text { Excellence } \\
\text { Grants } \\
\text { (Financial } \\
\text { Resource) } \\
\end{array}$ & $\begin{array}{l}\text { No } \\
\text { One needs an } \\
\text { internal access } \\
\text { to review } \\
\text { reports on intra- } \\
\text { website }\end{array}$ & $\begin{array}{l}\text { Discrimination } \\
\& \text { Bias Report } \\
\text { system featured } \\
\text { on diversity } \\
\text { website }\end{array}$ \\
\hline
\end{tabular}


CDO/Diversity Office Website Morrill University

\begin{tabular}{|c|c|c|c|c|}
\hline $\begin{array}{l}\text { Title of the } \\
\text { CDO } \\
\text { Report Line }\end{array}$ & $\begin{array}{c}\text { Statement on } \\
\text { what the CDO } \\
\text { Office Does }\end{array}$ & $\begin{array}{c}\text { Diversity } \\
\text { Strategic Plan }\end{array}$ & $\begin{array}{c}\text { Organizational } \\
\text { Chart }\end{array}$ & Definitions \\
\hline $\begin{array}{l}\text { Vice President } \\
\text { \& Associate } \\
\text { Provost of } \\
\text { Diversity } \\
\text { Provost } \\
\text { Dotted line } \\
\text { President }\end{array}$ & $\begin{array}{l}\text { Yes } \\
\text { Includes a } \\
\text { message from } \\
\text { the CDO }\end{array}$ & $\begin{array}{l}\text { Yes } \\
\text { Site also } \\
\text { includes } \\
\text { updates and } \\
\text { associated } \\
\text { timelines }\end{array}$ & Yes & $\begin{array}{l}\text { Yes } \\
\text { Explains the } \\
\text { campus' } \\
\text { definition of } \\
\text { diversity and } \\
\text { it's conceptual } \\
\text { framework for } \\
\text { diversity }\end{array}$ \\
\hline $\begin{array}{c}\text { Programs \& } \\
\text { Services }\end{array}$ & $\begin{array}{c}\text { Letter/ } \\
\text { Statement } \\
\text { from Provost - } \\
\text { President }\end{array}$ & Resources & $\begin{array}{c}\text { Campus } \\
\text { Statistics / } \\
\text { Campus } \\
\text { Studies } \\
\text { Findings }\end{array}$ & Other \\
\hline $\begin{array}{l}\text { Yes } \\
\text { Difficult } \\
\text { Dialogues } \\
\text { Series } \\
\text { Enhancing } \\
\text { Diversity } \\
\text { Speaker Series }\end{array}$ & No & $\begin{array}{l}\text { Yes } \\
\text { Funding } \\
\text { Resources } \\
\text { Programmatic } \\
\text { and support } \\
\text { resources for } \\
\text { students, staff, } \\
\text { faculty }\end{array}$ & \begin{tabular}{|l} 
Yes \\
CDO website \\
includes \\
campus \\
compositional \\
data statistics \\
and findings \\
from climate \\
assessments
\end{tabular} & \begin{tabular}{|l} 
Yes \\
Meditation \\
services can be \\
arranged out of \\
this office - \\
mediators are \\
distributed \\
across campus \\
- professionals \\
trained in \\
mediation \\
\\
Bias reporting \\
system is listed \\
on website
\end{tabular} \\
\hline
\end{tabular}


CDO/Diversity Office Website Central University

\begin{tabular}{|c|c|c|c|c|}
\hline $\begin{array}{l}\text { Title of the } \\
\text { CDO } \\
\text { Report Line }\end{array}$ & $\begin{array}{c}\text { Statement on } \\
\text { what the CDO } \\
\text { Office Does }\end{array}$ & $\begin{array}{c}\text { Diversity } \\
\text { Strategic Plan }\end{array}$ & $\begin{array}{c}\text { Organizational } \\
\text { Chart }\end{array}$ & Definitions \\
\hline $\begin{array}{l}\text { Vice Provost } \\
\text { for Diversity \& } \\
\text { Climate } \\
\text { Provost }\end{array}$ & $\begin{array}{l}\text { No. Website } \\
\text { focuses on the } \\
\text { campus' entire } \\
\text { diversity efforts } \\
\text { rather than the } \\
\text { CDO's office } \\
\text { A link includes } \\
\text { a listing of } \\
\text { diversity } \\
\text { officers } \\
\text { focusing on } \\
\text { students, these } \\
\text { diversity } \\
\text { officers are } \\
\text { spread } \\
\text { throughout the } \\
\text { campus by } \\
\text { organizational } \\
\text { divisions and } \\
\text { schools }\end{array}$ & $\begin{array}{l}\text { Site also } \\
\text { includes } \\
\text { information on } \\
\text { current process } \\
\text { for writing new } \\
\text { diversity plan } \\
\text { as well as lists } \\
\text { former diversity } \\
\text { plans going } \\
\text { back 30 years. } \\
\text { Includes } \\
\text { system-wide } \\
\text { plans as well. }\end{array}$ & No & No \\
\hline $\begin{array}{c}\text { Programs \& } \\
\text { Services }\end{array}$ & $\begin{array}{c}\text { Letter/ } \\
\text { Statement } \\
\text { from Provost - } \\
\text { President }\end{array}$ & Resources & $\begin{array}{c}\text { Campus } \\
\text { Statistics / } \\
\text { Campus } \\
\text { Studies } \\
\text { Findings } \\
\end{array}$ & Other \\
\hline $\begin{array}{l}\text { Yes } \\
\text { Programs, } \\
\text { departments, } \\
\text { and services are } \\
\text { listed - } \\
\text { however the } \\
\text { majority focus } \\
\text { on students. } \\
\text { Fewer } \\
\text { resources for } \\
\text { faculty \& staff }\end{array}$ & Yes & $\begin{array}{l}\text { Yes } \\
\text { The campus } \\
\text { offers an annual } \\
\text { symposiums } \\
\text { intended for } \\
\text { faculty, staff, } \\
\text { and students } \\
\text { focusing on } \\
\text { diverse topics }\end{array}$ & $\begin{array}{l}\text { Yes } \\
\text { There is a tab } \\
\text { that contains } \\
\text { reports related } \\
\text { to Central } \\
\text { University } \\
\text { diversity plans } \\
\text { from system, } \\
\text { campus, and } \\
\text { division }\end{array}$ & $\begin{array}{l}\text { Yes } \\
\text { Information on } \\
\text { how to } \\
\text { financially } \\
\text { contribute to } \\
\text { certain } \\
\text { programs and } \\
\text { scholarships }\end{array}$ \\
\hline
\end{tabular}


Appendix E

Interview Guides 


\section{Chief Diversity Officer Interview Guide}

1. Your campus was identified by members of the National Association of Diversity Officers in Higher Education (NADOHE) as being successful in its efforts to institutionalizing diversity. Do you think your campus has been successful? If yes, what key strategies contribute to your success? If you think your campus has not been successful, why is that?

2. What role do you play in institutionalizing diversity at your campus?

3. What outcome measures do you use to determine if the campus' efforts have been successful at institutionalizing diversity? What is your role in maintaining and tracking these efforts?

4. How are these efforts sustained? What is your role in these efforts? Who else is involved?

5. What was the campus' motivation for creating a chief diversity officer position?

6. When was this position created?

7. How long have you served in this role? Were you the inaugural person to hold this position?

8. What is your reporting line?

9. How is your relationship with the president and provost? Do you have open communication? Do you feel you have institutional authority to complete your job?

10. What are your campus' greatest challenges at institutionalizing diversity?

11. What else would you like to share about your campus institutionalization efforts or the chief diversity officer? 


\section{Faculty/Administrator Interview Guide}

1. Please tell me your position and/or work within the university and how that is related to the campus' diversity efforts?

2. Your campus was identified by members of the National Association of Diversity Officers in Higher Education (NADOHE) as being successful in its efforts to institutionalizing diversity. Do you think your campus has been successful? If yes, what key strategies do you think contribute to the campus' success? If you think your campus has not been successful, why is that?

3. What role do you think the chief diversity officer plays in institutionalizing diversity at your campus?

a. Do you interact with this person? If so, in what capacity?

4. From where you sit, what outcome measures do you look at to see if the campus has been successful at institutionalizing diversity? Are these the same measures the campus' diversity office uses?

5. Are you involved in contributing to these outcome measurers? If so, how?

6. How are the campus' diversity efforts sustained? What is your role in sustaining these efforts?

7. Are you supportive of the chief diversity officer position being a key leader in institutionalizing diversity at this campus? Why or why not?

8. What are your campus' greatest challenges at institutionalizing diversity campuswide?

9. Has the institutional mission and culture of the campus helped or hindered your diversity efforts?

10. What else would you like to share about your campus institutionalization efforts or the chief diversity officer? 


\section{Provost Interview Guide}

1. Your campus was identified by members of the National Association of Diversity Officers in Higher Education (NADOHE) as being successful in its efforts to institutionalizing diversity. Do you think your campus has been successful? If yes, what key strategies contribute to your success? If you think your campus has not been successful, why is that?

2. What role does the chief diversity officer play in institutionalizing diversity at your campus?

3. What outcome measures do you use to determine if your efforts have been successful at institutionalizing diversity?

4. How are these efforts sustained? What is the role of the chief diversity officer in these efforts? Who else is involved?

5. Did you hire the current chief diversity officer?

6. What was the campus' motivation for creating a chief diversity officer position? Are you supportive of the position?

7. When was the position created?

8. What are your campus' greatest challenges at institutionalizing diversity?

9. How has the institutional mission and culture of the campus helped or hindered your diversity efforts?

10. What else would you like to share about your campus institutionalization efforts or the chief diversity officer? 


\section{Student Interview Guide}

\section{Opening}

1. Please tell me your position and/or work within the university and how that is related to the campus' diversity efforts?

2. As a student, what has been your overall impression regarding diversity, inclusion, and equity at this campus? Have you been pleased? Disappointed? Or have no real opinion? Why?

\section{Successful Characteristics}

1. Your campus was identified by members of the National Association of Diversity Officers in Higher Education (NADOHE) as being successful in its efforts to institutionalizing diversity. Do you think your campus has been successful at institutionalizing diversity? If yes, why? If no, why not?

2. What do you think (or would) makes your institution successful at institutionalizing diversity?

3. From your perspective, what are (or would be) the key strategies your campuses utilizes to institutionalize diversity?

\section{Outcome Measures}

1. How would you measure success in the area of institutionalizing diversity? Or... What does success look like to you in terms of diversity and inclusion?

2. As a student, do you have a role in the campus' diversity efforts?

\section{Chief Diversity Officers}

1. As a student, are you aware that the campus has a chief diversity officer, charged with leading the campus strategic diversity efforts? If yes, do you work or interact with the $\mathrm{CDO}$ ? If yes, in what capacity?

2. [If Yes] Do you feel that the Chief Diversity Officers plays a key role in the institutionalization effort and your campus' success? If so, why? If no, why not?

3. Is it important for a campus to have a chief diversity officer to hold the university accountable for its diversity efforts? Why?

\section{Other}

1. As a student, is there anything else you would like to share regarding this topic? 
Appendix F

Interview Solicitation 
Greetings

I am a doctoral student at the University of San Diego conducting research on chief diversity officers in higher education and their role in institutionalizing diversity at their campuses. According to your peers that are members of the National Association of Diversity Officers in Higher Education (NADOHE), your university was identified as an institution that has been successful at institutionalizing diversity or is close to reaching that goal. As such, I would like to visit your campus and conduct qualitative interviews with you. Participation in this study involves an interview with me that will take no more than one hour of your time. Questions pertain to your campus diversity efforts and your apparent success. Data collected will be kept confidential. Participation is voluntary, but it is hoped you will take a moment of your valuable time to participate in this study in order to advance the research on chief diversity officers in higher education.

I will be in touch by phone in a week to inquire about your interest and availability.

If you have any questions, please contact me at cdavalos@sandiego.edu or inquire with my advisor, Dr. Cheryl Getz at cgetz@sandiego.edu.

Thank you in advance,

Cynthia Dávalos

Doctoral candidate, Leadership Studies

University of San Diego, School of Leadership \& Education Sciences 
Appendix G

Participation Consent Form 


\section{University of San Diego \\ Institutional Review Board}

\section{Research Participant Consent Form}

For the research study entitled:

EXPLORING THE RELATIONSHIP BETWEEN THE INSTITUTIONALIZATION OF DIVERSITY AND CHIEF DIVERSITY OFFICERS

\section{Purpose of the research study}

Cynthia Davalos is a student in the School of Leadership and Education Sciences (SOLES) at the University of San Diego. You are invited to participate in a research study he/she is conducting. The purpose of this research study is: to explore the relationship between institutional diversity efforts and chief diversity officers in higher education.

\section{What you will be asked to do}

If you decide to be in this study, you will be asked to:

Participate in a private interview about your knowledge of the campus' diversity efforts.

You will be audiotaped during the interview.

Your participation in this study will take a total of 60 minutes.

\section{Foreseeable risks or discomforts}

a) This study involves no more risk than the risks you encounter in daily life.

\section{Benefits}

While there may be no direct benefit to you from participating in this study, the indirect benefit of participating will be knowing that you helped researchers better understand the relationship between chief diversity officers and the institutionalization of diversity on university campuses.

\section{Confidentiality}

Any information provided and/or identifying records will remain confidential and kept in a locked file and/or password-protected computer file in the researcher's office for a minimum of five years. All data collected from you will be coded with a number or pseudonym (fake name). Your real name will not be used. The results of this research project may be made public and information quoted in professional journals and meetings, but information from this study will only be reported as a group, and not individually. 


\section{Compensation}

a) You will receive no compensation for your participation in the study.

\section{Voluntary Nature of this Research}

Participation in this study is entirely voluntary. You do not have to do this, and you can refuse to answer any question or quit at any time. Deciding not to participate or not answering any of the questions will have no effect on any benefits you're entitled to, like your health care, or your employment or grades. You can withdraw from this study at any time without penalty.

\section{Contact Information}

If you have any questions about this research, you may contact either:

\section{1) Cynthia Dávalos}

Email: cdavalos@sandiego.edu

Phone: 714-496-1497

\section{2) Dr. Cheryl Getz}

Email: cgetz@sandiego.edu

Phone: 619-260-4289

I have read and understand this form, and consent to the research it describes to me. I have received a copy of this consent form for my records.

Name of Participant (Printed) 